\title{
Catalogue of type specimens of fishes in the Western Australian Museum (Second Edition)
}

\author{
Glenn I. Moore ${ }^{1,2}$, J. Barry Hutchins, Kevin N. Smith and Susan M. Morrison ${ }^{3}$ \\ Fish Section, Department of Aquatic Zoology, \\ Western Australian Museum, 49 Kew St, Welshpool \\ Western Australia, 6106, Australia \\ ${ }^{1}$ current address: Department of Biology, Murdoch University, Centre for Fish and Fisheries Research, \\ Murdoch Unversity, South St, Murdoch, Western Australia, 6150, Australia \\ ${ }^{2}$ E-mail: g.moore@murdoch.edu.au. \\ ${ }^{3}$ E-mail: sue.morrison@museum.wa.gov.au
}

\begin{abstract}
The fish collection at the Western Austalian Museum contains type specimens of 704 species, of which 347 are primary types. A complete checklist is presented, including remarks on the current status of this material, synonyms, and a full bibliography. This revised catalogue supersedes the previous catalogue.
\end{abstract}

\section{INTRODUCTION}

Prior to this catalogue, lists of fish types in the Western Australian Museum have been published on three occasions (McKay 1965, 1966a; Hutchins and Smith 1991). The first two treatments include only primary types, covering 53 species. The last was comprehensive, including 400 species of both primary and secondary types and consideration of supposed types considered to be invalid. The last catalogue provides much of the basis for the present inventory, which lists 704 species with both primary and secondary types and invalid types. This revised catalogue presents an updated list and includes additions, omissions and corrections to Hutchins and Smith (1991), and should be considered as superseding the previous catalogue.

The Western Australian Museum's fish collection was commenced in 1896 as part of a larger multidisciplinary collection. Although the accession of specimens was at first slow, the fish collection has grown into an extensive assemblage of some 170,000 registered specimens. This is reflected in the number of type specimens now representing a total of 704 species, of which 347 are primary types. Many more manuscript names for specimens housed at the Western Australian Museum are currently awaiting publication.

Although the first descriptions of new fish species in the collections of the Western Australian Museum were published more than a hundred years ago, the period of greatest activity has been the past 30 years when the then Department of Fishes was created. Prior to this, the responsibility for fishes had been with various curators whose main interests were with other vertebrate and invertebrate groups (see historical section, below).
The responsibility for the fish collection now rests with the Fish Section within the Department of Aquatic Zoology.

Current nomenclatural status and synonymy follows Eschmeyer et al. (2007). The order of presentation is based on Yearsley et al. (1997). For simplicity, measurements are given to nearest millimetre. Readers should refer to original description for more precision. The following abbreviations are used in the text:

Specimens: c/s - cleared and stained; skel. skeletal material; SL - standard length; TL - total length.

Locations: NSW - New South Wales; NT Northern Territory; PNG - Papua New Guinea; Qld. - Queensland; SA - South Australia; Tas. Tasmania; Vic. - Victoria; WA - Western Australia; $\mathrm{N}$ - north; E - east; S - south; W - west (and combinations of these).

Institutions: are abbreviated as recommended by Leviton et al. (1985).

\section{HISTORY OF THE WESTERN AUSTRALIAN MUSEUM'S FISH COLLECTION}

The collections of the Western Australian Museum were initiated by the first curator B.H. Woodward in 1896. Over the next decade and a half, a growing number of natural history specimens was accessioned into the collection employing various catalogues. These included the Western Australian Museum Register (1896-1900), the Taxidermists Book (1900-1907), the Zoological Register (1907-1912), and the Catalogue of the Museum (1896-1912) (a register of specimens on display, and not official registration numbers). The 
fishes registered in these catalogues were each given a single number, some of which were prefixed by the letter ' $U$ '. A separate Fish Catalogue was eventually commenced in 1912 by the assistant in natural history, W.B. Alexander. All numbers in this catalogue were prefixed by the letter ' $P$ ' (abbreviation for Pisces), a system that is still in use today. In addition, between 1966 and 1971, a Fish Osteological Catalogue was used to register skeletal material using the prefix ' $\mathrm{PO}$ ' (abbreviation for Pisces Osteological).-This-system is no longer in use. Much of the fish material that was registered prior to 1912, and those registered under the 'PO' system, has since been re-registered using the ' $\mathrm{P}$ ' numbering system, and none of the earlier systems are now recognised.

In 1974, the first curator to be wholly responsible for fishes, G.R. Allen, introduced a refinement to Alexander's system. This incorporated a method of registration jointly developed by the fish departments at the Australian Museum and the Natural History Museum of Los Angeles County. Instead of allocating a separate number for each specimen, as was previously the case, all taxa with identical collection data are given the same ' $P$ ' number. Each separate species is then distinguished by the addition of a different three-digit suffix in the form of '-001', '-002', etc. Thus the ' $\mathrm{P}$ ' number component is a station number, which is further qualified by the addition of suffixes. Additional suffixes can be added when, for example, future studies show that specimens of two species were incorrectly lumped under the one species number. Specimens catalogued prior to 1974 have been incorporated into this system by, in most cases, the addition of '-001' (e.g. P.3442 is now P.3442-001). Therefore, in the list of type specimens presented below, each registration number includes a suffix, even though it may have originally been cited without this addition.

The collecting and subsequent accession of fish specimens for the Western Australian Museum collections can be divided into several periods. These are listed below, including the staff responsible and the dates for each period.

1. B.H. Woodward -1896 to 1912.

2. W.B. Alexander -1912 to 1920 .

3. L. Glauert - 1916 to 1957.

4. G.F. Mees - 1958 to 1963.

5. R.J. McKay - 1964 to 1972.

6. J.B. Hutchins -1972 to 2007.

7. G.R. Allen - 1974 to 1999.

8. S.M. Morrison - 2007 to present.

Most fish specimens are stored in glass jars in $70 \%$ ethanol, although larger specimens are kept in either plastic polydrums or vats. Some skeletal and cleared and stained material is also held, in addition to several mounted skins.

The majority of the collection comprises Western
Australian fauna, both marine and freshwater, but large numbers of freshwater species from Australia, Indonesia and Papua New Guinea are also present. Coral reef species from the Indo-West Pacific region are well represented, as is the inshore trawl-caught fauna of the state. A growing number of deep-water fishes from beyond the continental shelf have been added to the collection in recent years.

The size of the type collection of the Western Australian Museum has increased considerably in the years since the last type catalogue (Hutchins and Smith 1991) was published (704 versus 400 -species). Much of this is due to increases in the number of types of the following families:

Pomacentridae, 81 spp. (50 in 1991); Gobiidae, 71 spp. (25); Pseudomugilidae (including Melanotaenidae - these two families are now recogised as separate), 51 spp. (34); Apogonidae, 43 spp. (13); Labridae, 40 spp. (17); Eleotridae, 36 spp. (17); Blenniidae, 17 spp. (12); Tripterygiidae, 17 spp. (4); Pseudochromidae, 14 spp. (3); Gobiesocidae, 10 spp. (3); Plotosidae, 10 spp. (3); Soleidae, 10 spp. (1).

Despite exhaustive searches and requests to other institutions, some type material could not be found. Where this occurred, it is noted in the relevant species accounts. If there is sufficient evidence to suggest the specimens are lost, this opinion is indicated by the statement 'missing, presumed lost'. If the specimens could not be traced, but there is no direct evidence suggesting they are lost, they are labelled as 'missing'. Many specimens within the Terapontidae were irretrievably damaged while on loan (see Hutchins and Smith 1991) and this is indicated in the species accounts.

\section{E.R. WAITE TYPES}

The first fish types deposited at the Western Australian Museum were ten specimens representing nine species described by Waite (1905) from Western Australian Museum material, including Catulus labiosus, Dipulus caecus, Synodus sageneus, Bramichthys woodwardi, Neatypus obliquus, Chaetodon assarius, Cynoglossus broadhursti, Chaetodermis maccullochi, and Pseudomonacanthus galii, but confusion still exists concerning the location of some of these specimens. In 1904, the director of the Western Australian Museum, B.H. Woodward, sent two lots of specimens to the Australian Museum for identification. E.R. Waite (Curator of Fishes at the Australian Museum) identified most of the material, noting that some species appeared to be new (Waite, in litt.). These new species were subsequently described in 1905 and supposedly returned that same year. A partial list of the returned specimens, including the types of four species, is on file at the Western Australian Museum. At least two syntypes were retained by 
the Australian Museum. The fate of the remaining types on their return to the Western Australian Museum, however, is open to question. Most of the specimens had originally been registered in the Taxidermists Book before the loans were sent to the Australian Museum (numbers 6808-6827 for the first lot, 7018-7034 for the second lot), and some of these numbers are mentioned in the correspondence between Waite and Woodward. Apparently, the types of the species described by Waite were subsequently registered in the Catalogue of the Museum, but not labelled as types. These registrations could not have been made on the immediate return of the specimens as they are scattered throughout the register. In 1965, McKay, while attempting to organise a type collection of Western Australian Museum fishes, re-registered specimens he regarded as being among the original material in the Fish Catalogue, annotating the old Catalogue of the Museum with the new ' $\mathrm{P}$ ' numbers. However, it appears that some of these may not have been Waite's types (see account of Catulus labiosus). Also the inclusion by McKay of the supposed type of Hoplegnathus woodwardi (Waite 1900) in this 1965 listing is obviously an error as the type is still at the Australian Museum (AMS I.4215). Furthermore, several of these specimens are now missing and are presumed lost.

\section{FIS ENDEAVOUR COLLECTION}

In April 1910, August 1911, September 1913 and February 1915, portions of the fish collections made by the FIS Endeavour, which at the time was conducting trawling operations off Australia's southern coastline, were deposited at the Western Australian Museum. Much of this Endeavour material formed the basis of descriptions by McCulloch (1911, 1914a, 1915), many of which involved new species. However, even though numerous specimens were entered into the Western Australian Museum catalogues as 'co-types', much of the evidence suggests that only two of the nine listed types are valid. According to an introductory note to Vol. 1 of the Endeavour Results, all types and a set of 'co-types' were retained by the Australian Museum; the remaining specimens were distributed to other Australasian State museums. The valid types are Caranx humerosus McCulloch, 1915 and Pempheris elongata McCulloch, 1911. The remaining specimens appear to represent some additional non-type material referred to in the relevant descriptions by $\mathrm{McCulloch}$ (1914a). Following is a list of these supposed types, preceded by their Western Australian Museum registration numbers and followed by their Endeavour number:

P.57-001, Plagiogenion macrolepis McCulloch, 1914a, 2 specimens, 209-213 mm SL, E.2332, E.2406.
P.60-001, Insidiator harrisii McCulloch, 1914a, 130 mm SL, E.3240.

P.65-001, Polypnus tridentifer McCulloch, 1914a, 3 specimens, 53-63 mm SL, E.3543.

P.68-001, Insidiator jugosus McCulloch, 1914a, 3 specimens, 126-172 mm SL, E.2597.

P.71-001, Centriscops cristatus McCulloch, 1914a, 2 specimens, 226-227 mm SL, E.2151.

P.73-001, Allocyttus verrucosus propinquus McCulloch, 1914a, $250 \mathrm{~mm}$ SL, E.3281.

P.274-001, Hoplostethus mediterraneus latus McCulloch, 1914a, 71 mm SL, E.2467.

\section{LIST OF TYPE SPECIMENS}

\section{Orectolobidae}

Orectolobus hutchinsi Last, Chidlow and Compagno, 2006: 35, figures 1, 2, 3, 4 .

Paratypes. P.4736-001, 2 specimens, 234-236 mm TL, Albany, WA, Australia; P.6702-001, $200 \mathrm{~mm}$ TL, Shark Bay, WA, Australia; P.6704-001, 202 mm TL, Shark Bay, WA, Australia; P.12021-001, $576 \mathrm{~mm}$ TL, Point Peron, WA, Australia; P.27880-001, 2 specimens, 246-341 mm TL, Bunkers Bay, WA, Australia; P.26620-001, 369 mm TL, Rottnest Island, WA, Australia; P.27731001, $905 \mathrm{~mm}$ TL, Ocean Reef, WA, Australia.

\section{Scyliorhinidae}

Asymbolus submaculatus Compagno, Stevens and Last, 1999: 8, figure 2.

PARATYPeS. P.711-001, $414 \mathrm{~mm}$ TL, Bald Island, WA, Australia; P.28483-001, $387 \mathrm{~mm}$ TL, Esperance, WA, Australia; P.29399-001, 414 mm TL, Bunbury, WA, Australia.

Atelomycterus fasciatus Compagno and Stevens, 1993: 149, figures $1,2,3,4,5 a, b, 6,7,8,10$.

HoLoTYPE. P.8629-001, 369 mm TL, Onslow, WA, Australia.

Paratype. P.23838-001, $251 \mathrm{~mm}$ TL, Exmouth Gulf, WA, Australia.

Catulus labiosus Waite, 1905: 57, figure 23.

HoLotype. Uncatalogued, $620 \mathrm{~mm}$ TL, Fremantle, WA, Australia (missing, presumed lost).

Neotype. P.13253-001, 482 mm TL, no data.

RemarKs. A neotype was designated by Séret (1990) because of the confusion surrounding the whereabouts of the holotype. The latter was originally sent to AMS where it was described and subsequently returned to WAM (see section on Waite types in Introduction). The specimen later referred to by numerous workers as the holotype (see Séret (1990) for references) was selected by Whitley (1934) when he redescribed and illustrated the species. This specimen was 
subsequently catalogued in 1965 as P.13253, but apparently was not remeasured. As it is only 482 $\mathrm{mm}$ TL - Waite gave a length of $620 \mathrm{~mm}$ TL in the original description - we agree with Séret that this specimen cannot be the holotype. Even though we have located several old unlabelled specimens of this species at WAM, none matches the length given by Waite. Séret's designation of the second specimen as a neotype was, however, not justified as a nomenclatural problem was not -involved_(see_Art. 75(b)), International Code of Zoological Nomenclature).

SYNONYMY. This species is now known as Aulohalaelurus labiosus.

Halaelurus boesemani Springer and D'Aubrey, 1972: 11, figure $1 d$.

Paratype. P.14836-001, 430 mm TL, Shark Bay, WA, Australia.

REMARKS. In the original description, only the holotype was identified as a type, however as data from other listed specimens were used in the description the other specimens are considered to be valid paratypes (P. Last, pers. comm.). One of these paratypes is WAM P.14836-001.

\section{Triakidae}

Fur ventralis Whitley, 1943a: 397.

HolOtYPe. P.2451-001, 1250 mm TL, Bunbury, WA, Australia.

PARATYPE. P.26679-001 (mounted skin), Houtman Abrolhos, WA, Australia.

REMARKs. Other paratypes were listed in the description, but no registration numbers were provided. The whereabouts of these other specimens is unknown.

SYNONYMY. This name is a junior synonym of Furgaleus macki (Whitley, 1943b).

Hemitriakis falcata Compagno and Stevens, 1993: 198, figures 1, 2, 3, 4, 5, 6a, c, e.

HoLotYPe. P.26271-001, $770 \mathrm{~mm}$ TL, $\mathrm{N}$ of Rowley Shoals, WA, Australia.

PARATYPe. P.26274-007, $258 \mathrm{~mm}$ TL, N of Port Hedland, WA, Australia.

\section{Carcharhinidae}

Galeolamina (Galeolamnoides) eblis Whitley, 1944a: 252, figure 1 .

PARATYPE. P.667-001 (mounted specimen, jaws separate), $935 \mathrm{~mm} \mathrm{TL}$, Coventry Reef, WA, Australia.

SYNONYMY. This name is a junior synonym of Carcharhinus obscurus (Lesueur, 1818).

Galeolamna fowleri Whitley, 1944a: 255, figures 2, 2a.
Holotype. P.2503-001 (jaws only), South Muiron Island, WA, Australia.

RemarKs. See Garrick (1982) for details on the confusion surrounding this species.

SYNONYMY. This name is a junior synonym of Carcharhinus amblyrhynchos (Bleeker, 1856).

Longmania calamaria Whitley, 1944a: 257, figure 4. HoLOTYPE. P.2526-001, Busselton, WA, Australia (missing, presumed lost).

REMARKS. According to Whitley (1944a), the holotype-consisted-of-a head-and tail only, which cannot be found at WAM, so this specimen is missing, presumed lost (see Garrick [1982] for further details).

SYNONYMY. This name is a junior synonym of Carcharhinus brevipinna (Müller and Henle, 1839).

\section{Narcinidae}

Narcine lasti de Carvalho and Séret, 2002: 396, figures 1, 2, 3, 4, 5, 6 .

Paratypes. P.26208-025, 4 specimens, 225-261 mm TL, NNW of Port Hedland, WA, Australia.

Narcine westraliensis McKay, 1966b: 73, figures 3, $5 a, 5 b, 6$.

Holotype. P.6963-001, 212 mm TL, Shark Bay, WA, Australia.

Paratypes. P.5027-001, $200 \mathrm{~mm}$ TL; P.6946-001, 209 mm TL; P.6947-001, 176 mm TL; P.6948-001, 212 mm TL; P.6949-001, 190 mm TL; P.6950-001, 268 mm TL; P.6951-001, 203 mm TL; P.6952-001, 189 mm TL; P.6953-001, 184 mm TL; P.6954-001, 186 mm TL; P.6955-001, 174 mm TL; P.6956-001, 187 mm TL; P.6957-001, 180 mm TL; P.6958-001, 195 mm TL; P.6959-001, 200 mm TL; P.6960-001, 110 mm TL; P.6961-001, 182 mm TL; P.6962-001, 201 mm TL; P.6964-001, 207 mm TL; P.6965-001, $160 \mathrm{~mm}$ TL; P.6966-001, 195 mm TL; P.6967-001, 184 mm TL; P.6968-001, 193 mm TL; P.6969-001, 157 mm TL; P.6970-001, 202 mm TL; P.6971-001, $204 \mathrm{~mm}$ TL; P.6972-001, $180 \mathrm{~mm}$ TL; P.6973-001, 117 mm TL; P.6974-001, 195 mm TL; P.6975-001, 193 mm TL; P.6996-001, 182 mm TL; P.6997-001, $197 \mathrm{~mm}$ TL; P.6998-001, 190 mm TL; P.6999-001, 188 mm TL; P.7003-001, 196 mm TL; P.7004-001, 189 mm TL; P.7005-001, 190 mm TL; P.7009-001, 198 mm TL; P.7016-001, 222 mm TL; P.7018-001, 238 mm TL; P.7031-001, 220 mm TL; P.7033-001, $195 \mathrm{~mm}$ TL; P.7035-001, $194 \mathrm{~mm}$ TL, all from Shark Bay, WA, Australia.

\section{Rajidae}

Pavoraja alleni McEachran and Fechhelm, 1982: 8, figures 7,8 .

Holotype. P.19118-001, 297 mm TL, Rowley Shoals, WA, Australia. 
Paratypes. P.8226-001, $159 \mathrm{~mm} \mathrm{TL}$, eastern Indian Ocean; P.19117-001, 278 mm TL, Rowley Shoals, WA, Australia.

\section{Urolophidae}

Trygonoptera ovalis Last and Gomon, 1987: 63, figure 1.

Paratypes. P.27037-001, $151 \mathrm{~mm}$ disc width, Quindalup, WA, Australia; P.27285-001, $160 \mathrm{~mm}$ disc width, Mandurah, WA, Australia; P.27585002, $141 \mathrm{~mm}$ disc width, Houtman Abrolhos, WA, Australia.

Trygonoptera personata Last and Gomon, 1987: 65, figure 2.

Paratypes. P.8194-001, $165 \mathrm{~mm}$ disc width, Cottesloe Bank, WA, Australia; P.12034-001, 173 mm disc width, Shark Bay, WA, Australia; P.14104-001, 193 mm disc width, Rottnest Island, WA, Australia.

Urolophus circularis McKay, 1966b: 69, figure 2. HoLOTYPE. P.8191-001, 478 mm TL, Carnac Island, WA, Australia.

Urolophus lobatus McKay, 1966b: 71.

HoLotYPE. P.14133-001, 205 mm TL, Rottnest Island, WA, Australia.

ParatyPes. P.14120-001, 206 mm TL; P.14121-001, $201 \mathrm{~mm}$ TL; P.14122-001, 260 mm TL; P.14123001, $225 \mathrm{~mm}$ TL; P.14124-001, $251 \mathrm{~mm}$ TL; P.14125-001, $188 \mathrm{~mm}$ TL; P.14126-001, $260 \mathrm{~mm}$ TL; P.14127-001, 202 mm TL; P.14129-001, 204 mm TL; P.14130-001, 250 mm TL; P.14131-001, $212 \mathrm{~mm}$ TL; P.14134-001, 223 mm TL; P.14135001, $208 \mathrm{~mm}$ TL; P.14136-001, $197 \mathrm{~mm} \mathrm{TL;}$ P.14137-001, $288 \mathrm{~mm}$ TL; P.14138-001, $239 \mathrm{~mm}$ TL; P.14140-001, 198 mm TL, all from Rottnest Island, WA, Australia.

Urolophus westraliensis Last and Gomon, 1987: 72, figure 6.

Paratypes. P.26193-006, $225 \mathrm{~mm}$ disc width, Northwest Shelf, WA, Australia; P.26262-007, $182 \mathrm{~mm}$ disc width, $\mathrm{W}$ of Buccaneer Archipelago, WA, Australia.

\section{Muraenidae}

Gymnothorax woodwardi McCulloch, 1912: 80, figure 1 .

Holotype. P.13263-001, $515 \mathrm{~mm}$ TL, Pelsart Island, WA, Australia.

\section{Congridae}

Gnathophis melanocoelus Karmovskaya and Paxton, 2000: S12, figure 5d.

Holotype. P.7435-001, $238 \mathrm{~mm}$ TL, Rottnest Island, WA, Australia.
Paratypes. P.7436-001, $214 \mathrm{~mm}$ TL; P.7437-001, $230 \mathrm{~mm}$ TL; P.7438-001, $232 \mathrm{~mm} \mathrm{TL}$, all from Rottnest Island, WA, Australia.

REMARKS. There is considerable confusion with the registration numbers used in the type description. Originally 11 WAM specimens were examined, P.7435-001 to P.7445-001. This number series was presented as P.7435-7445 without suffixes for both the holotype and five paratypes. The holotype is P.7435-001 and the three WAM paratypes are P.7436-001, P.7437-001 and P.7438-001. The two remaining paratypes, originally registered as P.7439-001 and P.7440001 were donated to AMS (now I.40431-001) and ZMMU (registration number unknown) respectively. The other five specimens, P.7441001 to P.7445-001 were not listed and are nontypes.

Gnathophis microps Karmovskaya and Paxton, 2000: S10, figure $5 \mathrm{~b}$.

Paratype. P.28056-005, 365 mm TL, Rowley Shoals, WA, Australia.

Gnathophis nasutus Karmovskaya and Paxton, 2000: S11, figure 5c.

Paratype. P.31739-001, $188 \mathrm{~mm}$ TL, Port Hedland, WA, Australia.

Remarks. The type description listed AMS I.22875-001 as comprising 5 paratypes, 169-188 $\mathrm{mm}$ TL. Of these, 3 specimens ( $169-175 \mathrm{~mm} \mathrm{TL})$ were retained by AMS one the remaining transferred from AMS to WAM (P.31739-001, $188 \mathrm{~mm} \mathrm{TL}$ ) and ZMMU (20666, no measurement).

Gorgasia galzini Castle and Randall, 1999: 34, figure 22.

Paratypes. P.28540-011, 5 specimens, 232-385 $\mathrm{mm}$ TL, Holmes Reef, Qld., Australia

Heteroconger perissodon Böhlke and Randall, 1981: 377 , figures 5 right, 7 .

Paratype. P.26788-001, $324 \mathrm{~mm}$ TL, Ambon Island, Indonesia.

Leptocephalus wilsoni hesperius Whitley, 1944b: 25 , figure 1.

HOLOTYPE. P.2603-001, 464mm TL, Leighton, WA, Australia.

SYNONYMY. This name is a junior synonym of Conger wilsoni (Bloch and Schneider, 1801).

\section{Ophichthidae}

Phyllophichthus macrurus McKay, 1970: 4. HoLotyPe. P.4015-001, 465 mm TL, Kalgan River, WA, Australia.

Paratypes. P.16789-001, $401 \mathrm{~mm}$ TL, Bernier Island, WA, Australia; P.16790-001, 346 mm TL, Jurien Bay, WA, Australia. 
Scolecenchelys tasmaniensis smithi Whitley, 1944a: 262.

Holotype. P.704-001, 275 mm TL, Quindalup, WA, Australia.

Paratype. P.1016-001, 350 mm TL, Rottnest Island, WA, Australia.

SYNONYMY. This name is a junior synonym of Scolecenchelys tasmaniensis (McCulloch, 1911).

\section{Clupeidae}

Fluvialosa bulleri Whitley, 1948: 267.

Holotype. P.2945-001, $120 \mathrm{~mm}$ SL, Ord River, WA, Australia.

SYNONYMX. This name is a junior synonym of Nematalosa erebi (Günther, 1868).

Fluvialosa paracome Whitley, 1948: 267. Holotype. P.2619-001, 97 mm SL, Fitzroy River, WA, Australia.

SYNONYMY. This name is a junior synonym of Nematalosa erebi (Günther, 1868).

Herklotsichthys collettei Wongratana, 1987: 105, figure 1.

Holotype. P.25464-004, 108 mm SL, Shark Bay, WA, Australia.

PARATYPes. P.2544-001, 59 mm SL, Onslow, WA, Australia; P.2545-001, $67 \mathrm{~mm}$ SL, Onslow, WA, Australia; P.22574-001, 98 mm SL, Shark Bay, WA, Australia; P.23870-001, 97 mm SL, Exmouth Gulf, WA, Australia; P.25464-002, 6 specimens, 100-107 mm SL, Shark Bay, WA, Australia; P.26667-001, $43 \mathrm{~mm}$ SL, Shark Bay, WA, Australia; P.27380-001, 91 mm SL, Shark Bay, WA, Australia.

\section{Engraulididae}

Setipinna paxtoni Wongratana, 1987: 109, figure 5. HolotyPe. P.16267-001, 110 mm SL, Cambridge Gulf, WA, Australia.

PARATYPES. P.16268-001, 80 mm SL; P.16269-001, $94 \mathrm{~mm}$ SL; P.16270-001, $109 \mathrm{~mm}$ SL, all from Cambridge Gulf, WA, Australia.

REMARKS. The type description includes two errors: the holotype P.16267-100 should read P.16267-001 and the paratypes P.16268-70-001 should read P.16268-001, P.16269-001 and P.16270-001.

\section{Galaxiidae}

Galaxias pusillus nigrostriatus Shipway, 1953: 175, figure.

HoLotyPE. P.3420-001, 28 mm SL, Marbellup Creek, WA, Australia.

SYNONYMY. This species is now known as Galaxiella nigrostriata.
Galaxias truttaceus hesperius Whitley, 1944a: 263. HolotyPe. P.2580-001, 125 mm SL, Taylor's Inlet, WA, Australia.

SYNONYMY. This name is a junior synonym of Galaxias truttaceus Valenciennes, 1846.

Galaxiella munda McDowall, 1978: 119, figures 1, 2. HolotYPE. P.25736-001, 38 mm SL, Scott River, WA, Australia.

PARATYPes. P.5225-001, 39 specimens, 23-42 mm SL, Scott River, WA, Australia.

\section{Lepidogalaxiidae}

Lepidogalaxias salamandroides Mees, 1961: 33, figure 1 .

HoLotyPe. P.4887-001, 49 mm SL, Shannon River, WA, Australia.

PARATYPes. P.4888-001, 3 specimens, 32-42 mm SL, Shannon River, WA, Australia.

\section{Sternoptychidae}

Maurolicus javanicus Parin and Kobyliansky, 1993: 100.

PARATYPES. P.15594-001, $28 \mathrm{~mm}$ SL, west of North West Cape, WA, Australia; P.15595-001 to P.15601-001, 7 specimens, 27-34 mm SL, all from west of Cape Cuvier, WA, Australia.

REMARKS. The type description includes an error: the paratype P.15595 should read P.15594-001.

\section{Synodontidae}

Synodus sageneus Waite, 1905: 58, Plate 8, figure 1. HoLotYPe. P.13254-001, $246 \mathrm{~mm}$ SL, between Fremantle and Houtman Abrolhos, WA, Australia.

REMARKS. This specimen has been allocated two different WAM registration numbers. It was first registered as P.13254-001 in 1965. In 1976, it was again registered as P.25509-001 as the specimen appeared to be unregistered (a paper label attached to the specimen had disintergrated). Although the latter number has been referred to in at least two papers (Cressey 1981; Paxton et al. 1989), the original number, as reported by McKay (1966a), is retained.

\section{Cobitidae}

Cobitis aurata vallachia Nalbant, 1957: 209, figure 1. Paratypes. P.5938-001, 4 specimens, $42-60 \mathrm{~mm}$ SL, Ialomitza River, Romania.

SYNONYMY. This species is now known as Sabanejewia aurata vallachia. 


\section{Ariidae}

Arius coatesi Kailola, 1990: 18, figure 5.

Paratypes. P.28221-001， 3 specimens, 290-390 mm SL, Angoram, PNG.

REMARKS. The type description includes an error: for the paratypes P.28221-001 there are 3 specimens, not 4.

SYNONYMY. This species is now known as Ariopsis coatesi.

Arius hardenbergi Kailola, 2000: 137, figures 7, 8. HoLotyPe. P.29966-001, 260 SL, Manimeri River, West Papua, Indonesia, Indonesia.

SynONYMY. This species is now known as Apistor hardenbergi.

Arius midgleyi Kailola and Pierce, 1988: 75, figures $1,2,5,6$.

Paratypes. P.21338-002, 4 specimens, 133-161 mm SL, Ord River, WA, Australia; P.25597-001, $348 \mathrm{~mm}$ SL, Kimberley Region, WA, Australia; P.25708-001, $224 \mathrm{~mm} \mathrm{SL,} \mathrm{Forbes} \mathrm{Beach,} \mathrm{WA,}$ Australia; P.28776-001 (c/s), 166 mm SL, Lake Argyle, WA, Australia.

SYNONYMY. This species is now known as Ariopsis midgleyi.

Arius utarus Kailola, 1990: 12, figure 4.

ParatyPes. P.27846-001, 4 specimens, 97-133 mm SL, Sepik River, PNG; P.28224-001, 252 mm SL, Sepik River, PNG; P.27847-010, 7 specimens, 84118 mm SL, Kwatit River, PNG.

SYNONYMY. This species is now known as Ariopsis utarus.

\section{Plotosidae}

Euristhmus microphthalmus Murdy and Ferraris, 2006: 84, figures $1 \mathrm{~A}, 2 \mathrm{C}, 7$.

Paratypes. P.29959-003, 270 mm SL, Bintuni Bay, West Papua, Indonesia; P.29974-047, 201mm SL, Bintuni Bay, West Papua, Indonesia.

Euristhmus sandrae Murdy and Ferraris, 2006: 88, figures $2 \mathrm{E}, 10$.

HolotyPe. P.32730-001, $148 \mathrm{~mm}$ SL, Exmouth Gulf, WA, Australia.

Neosiluroides cooperensis Allen and Feinberg, 1998: 12, figure 2.

Paratypes. P.26436-001, 3 specimens, 179-213 mm SL, Cooper Creek, SA, Australia.

Neosilurus gloveri Allen and Feinberg, 1998: 13, figure 3.

Paratypes. P.29797-001, 15 specimens, 40-84 mm SL, Dalhousie Springs, SA, Australia.

Neosilurus mollespiculum Allen and Feinberg, 1998: 16, figure 6.
Paratypes. P.30544-002, $345 \mathrm{~mm}$ SL, Fanning River, Qld., Áustralia; P.30545-001, 3 specimens, 144-203 mm SL; P.30546-001, $148 \mathrm{~mm} \mathrm{SL;}$ P.30548-002, 2 specimens, 39-114 mm SL; P.30559-002, 3 specimens, $123-160 \mathrm{~mm} \mathrm{SL}$, all from Burdekin River, Qld., Australia

Neosilurus pseudospinosus Allen and Feinberg, 1998: 15, figures 4, 5 .

HoLotype. P.28505-007, 333 mm SL, Ord River, WA, Australia.

Paratypes. P.25404-007, 11 specimens, 56-153 mm SL, Morgunda Creek, WA, Australia; P.25406-006, $135 \mathrm{~mm}$ SL, Morgunda Creek, WA, Australia; P.25410-007, $130 \mathrm{~mm} \mathrm{SL}$, Drysdale River, WA, Australia; P.25413-005, 2 specimens, $90-97 \mathrm{~mm} \mathrm{SL}$, Orchid Creek, WA, Australia; P.25420-006, 5 specimens, 89-147 mm SL, Palmoondoora Creek, WA, Australia; P.25456-011, 238 mm SL, Drysdale River, WA, Australia; P.25732-001, 21 specimens, 60-119 $\mathrm{mm}$ SL, Bow River, WA, Australia; P.25872-005, $280 \mathrm{~mm}$ SL, Manning Creek Gorge, WA, Australia; P.25876-002, 2 specimens, 127-133 mm SL, Durack River, WA, Australia; P.28505006, 3 specimens, $102-325 \mathrm{~mm}$ SL, Ord River, WA, Australia.

REMARKS. The type description includes an error: for the paratypes P.28505-006 there are 3 specimens, not 8 .

Oloplotosus torobo Allen, 1985a: 248, figure 1.

Holotype. P.28158-003, $173 \mathrm{~mm}$ SL, Lake Kutubu, PNG.

Paraplotosus butleri Allen, 1998a: 129, figure 3. Holotype. P.27368-021, 325 mm SL, Broome, WA, Australia.

Paratypes. P.24084-001， $238 \mathrm{~mm}$ SL, Kendrew Island, WA, Australia; P.24270-001, 257 mm SL, Kendrew Island, WA, Australia; P.25108-022, $104 \mathrm{~mm}$ SL, Kendrew Island, WA, Australia; P.25117-006, $40.5 \mathrm{~mm}$ SL, Lady Nora Island, WA, Australia; P.25368-007, 80 mm SL, North West Cape, WA, Australia; P.25374-009, 5 specimens, 100-115 mm SL, North West Cape, WA, Australia; P.25711-001, 199 mm SL, North West Cape, WA, Australia (missing); P.25813013, 2 specimens, 79-111 mm SL, South Muiron Island, WA, Australia; P.27967-004, 90 mm SL, Point Quobba, WA, Australia; P.27980-013, 2 specimens, 168-281 mm SL, Monte Bello Islands, WA, Australia; P.28416-009, 5 specimens, 67-265 mm SL, Gantheume Point, WA, Australia; P.30847-014, $225 \mathrm{~mm} \mathrm{SL}$, Cassini Island, WA, Australia; P.31088-008, 4 specimens, $42-183 \mathrm{~mm}$ SL, Napier Broome Bay, WA, Australia; P.31091003, 3 specimens, 26-130 mm SL, Napier Broome Bay, WA, Australia. 
Tandanus bostocki Whitley, 1944a: 260.

HolotyPe. P.241-001, $370 \mathrm{~mm}$ SL, Serpentine, WA, Australia (invalid designation).

REMARKs. Whitley (1944) proposed Tandanus bostocki as a replacement name for Plotosus unicolor Castelnau, 1873 (preoccupied by $P$. unicolor Valenciennes, 1840), a freshwater species described from WA, Australia. Whitley presented a redescription of the species and nominated one of his specimens as the holotype. However,-under-the-provisions of International Code of Zoological Nomenclature (Article 72.7), the type of the species must remain with the extant syntype of $P$. unicolor (NMV A.11773, formerly registered as NMV 51851), one of Castelnau's two syntypes (the other is missing, presumed lost). Therefore the holotype cited above has no validity.

Tandanus coatesi Allen, 1985a: 252, figure 3.

HoLotyPe. P.27839-007, 139 mm SL, Ninar River, PNG.

Paratypes. P.27839-005, 50 specimens, 36-113 mm SL, Ninar River, PNG; P.27840-005, 68 mm SL, Lumi River, PNG.

\section{Batrachoididae}

Batrachoides waltersi Collette and Russo, 1981: 208, figure 5 .

Paratypes. P.26494-001, 2 specimens, 72-84 mm SL, Jiquilisco Bay, El Salvador

REMARKS. The type description includes an error: the paratypes P.2649-001 should read P.26494001.

Batrachomoeus occidentalis Hutchins, 1976: 23, figures $9,10$.

HoLOTYPE. P.23702-001, 169 mm SL, Exmouth Gulf, WA, Australia.

PARATYPES. P.9343-001, $32 \mathrm{~mm}$ SL, North West Cape, WA, Australia; P.23876-001, 113 mm SL, North West Cape, WA, Australia; P.23877-001, $131 \mathrm{~mm}$ SL (skel.), North West Cape, WA, Australia; P.25091-001, 32 mm SL, Green Head, WA, Australia; P.25092-001, 33 mm SL, Rottnest Island, WA, Australia; P.25093-001, 2 specimens, 73-104 mm SL (1 skel.), Rottnest Island, WA, Australia.

REMARKS. The type description lists paratypes P.23876-7. These specimens are now registered as P.23876-001 and P.23877-001.

Batrachomoeus rubricephalus Hutchins, 1976: 30, figures 12.

HolotYPE. P.25082-001, 211 mm SL, Garden Island, WA, Australia.

Paratypes. P.159-001, $196 \mathrm{~mm}$ SL (skel.), Eucla, WA, Australia; P.1195-001, 232 mm SL, Point Peron, WA, Australia; P.4003-001, 262 mm SL,
Rottnest Island, WA, Australia; P.4292-001, 203 mm SL, near Perth, WA, Australia; P.5792-001, $191 \mathrm{~mm}$ SL, Safety Bay, WA, Australia; P.6092001, $214 \mathrm{~mm}$ SL, Cervantes, WA, Australia; P.7120-001, $256 \mathrm{~mm} \mathrm{SL,} \mathrm{Lancelin} \mathrm{Island,} \mathrm{WA,}$ Australia; P.7122-001, $236 \mathrm{~mm}$ SL, Shoalwater Bay, WA, Australia; P.7198-001, 256 mm SL, Marmion, WA, Australia; P.14788-001, 193 mm SL, Houtman Abrolhos, WA, Australia; P.25083001, $222 \mathrm{~mm}$ SL (skel.), Recherche Archipelago, WA, Australia; P.25123-001, $111 \mathrm{~mm}$ SL, Cliff Head Point, WA, Australia.

Halophryne ocellatus Hutchins, 1974: 115, figures 1, 3a.

Holotype. P.5956-001, 190 mm SL, Houtman Abrolhos, WA, Australia.

PARATYPES. P.3364-001, $138 \mathrm{~mm}$ SL, Houtman Abrolhos, WA, Australia; P.5851-001, 2 specimens, 109-127 mm SL, Shark Bay, WA, Australia; P.5955-001, 102 mm SL, Shark Bay, WA, Australia; P.6149-001, 136 mm SL, Shark Bay, WA, Australia; P.6260-001, $130 \mathrm{~mm} \mathrm{SL}$, Shark Bay, WA, Australia; P.7148-001, $47 \mathrm{~mm}$ SL, Dampier Archipelago, WA, Australia; P.9393-001, 43 mm SL, Exmouth Gulf, WA, Australia; P.9394-001, 45 mm SL, Exmouth Gulf, WA, Australia; P.12073-001, 38 mm SL, Exmouth Gulf, WA, Australia; P.13767-001, 75 mm SL, Shark Bay, WA, Australia; P.13768-001, 98 mm SL, Shark Bay, WA, Australia; P.13769-001, 98 mm SL, Shark Bay, WA, Australia; P.14653-001, $101 \mathrm{~mm}$ SL, Shark Bay, WA, Australia; P.14833001, $124 \mathrm{~mm}$ SL, Shark Bay, WA, Australia; P.16296-001, $102 \mathrm{~mm} \mathrm{SL,} \mathrm{Exmouth} \mathrm{Gulf,} \mathrm{WA,}$ Australia; P.16483-001, 88 mm SL, Broome, WA, Australia; P.23604-001, $99 \mathrm{~mm}$ SL, Carnarvon, WA, Australia; P.24830-001, $23 \mathrm{~mm} \mathrm{SL}$, Carnarvon, WA, Australia; P.24562-001, $101 \mathrm{~mm}$ SL, Onslow area, WA, Australia; P.24829-001, 231 mm SL, Kalbarri, WA, Australia.

\section{Gobiesocidae}

Alabes brevis Springer and Fraser, 1976: 19.

Holotype. P.24385-001, 20 mm SL, Rottnest Island, WA, Australia.

Paratypes. P.24386-001, $16 \mathrm{~mm}$ SL, Rottnest Island, WA, Australia; P.24387-001, 23 mm SL, Rottnest Island, WA, Australia.

REMARKS. The type description lists paratypes P.24386-7. These specimens are now registered as P.24386-001 and P.24387-001.

Alabes elongata Hutchins and Morrison, 2004: 150, figure 2 .

HolotYPE. P.28296-003, 86 mm SL, Mondrain Island, WA, Australia.

PARATYPES. P.26004-012, 56 mm SL, Recherche Archipelago, WA, Australia; P.28280-002 (c/s), 
58 mm SL, Israelite Bay, WA, Australia; P.28296050, $92 \mathrm{~mm} \mathrm{SL}$, Mondrain Island, WA, Australia; P.32205-001, $42 \mathrm{~mm} \mathrm{SL}$, Cervantes, WA, Australia.

Alabes gibbosa Hutchins and Morrison, 2004: 151, figure 3.

HoLotyPe. P.27985-001, 67 mm SL, Carnac Island, WA, Australia.

Paratypes. P.32206-001, 91 mm SL, Safety Bay, WA, Australia; P.32207-001 (c/s), $71 \mathrm{~mm} \mathrm{SL}$, Carnac Island, WA, Australia; P.32208-001, 87 $\mathrm{mm}$ SL, Rottnest Island, WA, Australia; P.32209001, 84 mm SL, Cockburn Sound, WA, Australia. REMARKS. The type description includes an error: the year of collection for the holotype is 1983, not 1982.

Alabes occidentalis Hutchins and Morrison, 2004: 153, figure 4.

HoLotype. P.25465-001, 36 mm SL, Safety Bay, WA, Australia.

Paratypes. P.25465-004, 3 specimens, 22-40 mm SL, Safety Bay, WA, Australia; P.25805-002, 26 mm SL, Garden Island, WA, Australia; P.27952012, 5 specimens, 17-31 mm SL, Jurien Bay, WA, Australia; P.28267-001, 5 specimens, $34-38 \mathrm{~mm}$ SL, Carnac Island, WA, Australia; P.28280-001, 6 specimens (2 c/s), 27-35 mm SL, Israelite Bay, WA, Australia; P.28291-001, 3 specimens, 27-30 mm SL, Twilight Cove, WA, Australia; P.30384002, 4 specimens, 29-39 mm SL, Rottnest Island, WA, Australia.

Alabes scotti Hutchins and Morrison, 2004: 155, figure 5.

Paratype. P.32222-001 (c/s), 43 mm SL, Tathra, NSW, Australia.

Aspasmogaster occidentalis Hutchins, 1984: 131, figures 1, 3, 4, 5 .

HOLOTYPE. P.27882-001, 66 mm SL, Bunkers Bay, WA, Australia.

PARATYPes. P.25770-022, 3 specimens $(1 \mathrm{c} / \mathrm{s}), 22-$ $80 \mathrm{~mm}$ SL, Recherche Archipelago, WA, Australia; P.25774-008, $55 \mathrm{~mm}$ SL, Recherche Archipelago, WA, Australia; P.26000-013, $23 \mathrm{~mm}$ SL, Recherche Archipelago, WA, Australia; P.26002-007 (c/s), $25 \mathrm{~mm}$ SL, Recherche Archipelago, WA, Australia; P.26007-006, $70 \mathrm{~mm}$ SL, Recherche Archipelago, WA, Australia; P.26009-003, 68 mm SL, Recherche Archipelago, WA, Australia; P.26546-005, $62 \mathrm{~mm} \mathrm{SL}$, Saddle Island, WA, Australia; P.26620-004, 26 mm SL, Rottnest Island, WA, Australia; P.27880-003, 3 specimens, 67-93 mm SL, Bunkers Bay, WA, Australia.

Cochleoceps bassensis Hutchins, 1983: 40, figures $3 b, 4 b, 5,6,7$.
Paratype. P.27750-001 (c/s), $40 \mathrm{~mm}$ SL, Bass Strait, Tas., Australia

Cochleoceps bicolor Hutchins, 1991: 663, figures 4b, $5,6,7$.

Holotype. P.28522-002, $22 \mathrm{~mm} \mathrm{SL}$, Flinders Island, WA, Australia.

PARATYPES. P.25763-002, $13 \mathrm{~mm}$ SL, Recherche Archipelago, WA, Australia; P.28296-007, 7 specimens, 14-26 mm SL, Recherche Archipelago, WA, Australia; P.26608-025 (c/s), $20 \mathrm{~mm} \mathrm{SL,}$ Cheyne Beach, WA, Australia; P.28265-001, 30 mm SL, Rottnest Island, WA, Australia; P.28293007, 4 specimens, 15-22 mm SL, Lucky Bay, WA, Australia; P.28300-003, 4 specimens, $18-21 \mathrm{~mm}$ SL, Lucky Bay, WA, Australia.

Cochleoceps orientalis Hutchins, 1991: 668, figure 8. HoLOTYPE. P.27103-001, 22 mm SL, Big Island, NSW, Australia.

Paratypes. P.27103-002, 3 specimens $(1 \mathrm{c} / \mathrm{s}), 21-$ $23 \mathrm{~mm}$ SL, Big Island, NSW, Australia.

Cochleoceps viridis Hutchins, 1991: 656, figures 1, $2,3,4 a$.

HoLotyPe. P.26604-015, 39 mm SL, Albany, WA, Australia.

Paratypes. P.26455-011, 5 specimens, 14-21 mm SL, Geographe Bay, WA, Australia; P.26604-009, 6 specimens $(1 \mathrm{c} / \mathrm{s}), 30-36 \mathrm{~mm}$ SL, Albany, WA, Australia; P.26622-004, 5 specimens, 29-33 mm SL, Rottnest Island, WA, Australia; P.28267-003, 7 specimens, $23-27 \mathrm{~mm} \mathrm{SL}$, Carnac Island, WA, Australia; P.28280-004, 2 specimens, 33-33 mm SL, Israelite Bay, WA, Australia; P.28288-003, 39 $\mathrm{mm}$ SL, Israelite Bay, WA, Australia.

\section{Antennariidae}

Echinophryne glauerti Whitley, 1944a: 272.

HoLotype. P.1459-001, 131 mm SL, Cottesloe, WA, Australia.

Paratype. P.1232-001, $60 \mathrm{~mm}$ SL, Cottesloe, WA, Australia.

SYNONYMY. This species is now known as Allenichthys glauerti.

Lophiocharon hutchinsi Pietsch, 2004: 160, Plate 1. HoLotype. P.27673-002, 43 mm SL, James Price Point, WA, Australia.

Paratypes. P.24486-001, $33 \mathrm{~mm} \mathrm{SL}$, Exmouth Gulf, WA, Australia; P.28416-015, 2 specimens, 19-29 mm SL, Gantheume Point, WA, Australia; P.31884-001, $31 \mathrm{~mm}$ SL, Dampier Archipelago, WA, Australia.

Cheironectes trisignatus Richardson, 1844: 15, Plate 9 (figure 1).

Neotype. P.27274-013, 74 mm SL, Broome, WA, Australia. 
Remarks. Pietsch and Grobecker (1987) designated this neotype because of the confusion between this species - for which the type has been lost - and C. caudimaculatus Rüppell, 1829 which is a junior synonym of Antennarius commersoni (Latreille, 1804).

Synonymy. This species is now known as Lophiocharon trisignatus.

Rhycherus gloveri Pietsch, 1984: 70, figure 3b. Paratupes. P.3114-001, $115 \mathrm{~mm}$ SL, North Beach, WA, Australia; P.5814-001, 103, Warnboro Beach, WA, Australia; P.12111-001, $111 \mathrm{~mm} \mathrm{SL}$, Ledge Point, WA, Australia; P.2315-001, $87 \mathrm{~mm}$ SL, Cottesloe, WA, Australia; P.24867-001, 100 mm SL, Perth, WA, Australia.

\section{Moridae}

Eeyorius hutchinsi Paulin, 1986: 204, figure 2. HoLOTYPe. P.27128-001, $194 \mathrm{~mm}$ SL, Port Phillip Bay, Vic., Australia

ParatyPes. P.25781-002, $145 \mathrm{~mm}$ SL, Rottnest Island, WA, Australia; P.27123-001, $105 \mathrm{~mm} \mathrm{SL,}$ Wilson's Promontory, Vic., Australia; P.27126001, 2 specimens $(1 \mathrm{c} / \mathrm{s}), 87-209 \mathrm{~mm}$ SL, Wilson's Promontory, Vic., Australia (c/s specimen missing); P.27545-001, $178 \mathrm{~mm}$ SL, Port Esperance, Tas., Australia; P.27545-002, 3 specimens, $89-180 \mathrm{~mm}$ SL, Port Esperance, Tas., Australia; P.27549-006, 245 mm SL, Port Arthur, Tas., Australia; P.27564-003, 183 mm SL, Bridport, Tas., Australia; P.27616-003, $160 \mathrm{~mm}$ SL, Rottnest Island, WA, Australia.

\section{Ophidiidae}

Neobythites australiensis Nielsen, 2002: 20, figure 10.

HoLотYPE. P.28107-001, $230 \mathrm{~mm}$ SL, vicinity of Rowley Shoals, WA, Australia.

Paratypes. P.25395-008, 212 mm SL; P.25401-009, 195 mm SL; P.28107-002, 180 mm SL; P.30585$004,245 \mathrm{~mm}$ SL, all from vicinity of Rowley Shoals, WA, Australia.

Sirembo metachroma Cohen and Robins, 1986: 253, Plate $1 \mathrm{a}, \mathrm{b}$.

Paratype. P.25739-005, 140 mm SL, Moreton Bay, Qld., Australia

\section{Bythitidae}

Diancistrus alleni Schwarzhans, Møller and Nielsen, 2005: 91, figures 9, 10, 11.

Paratypes. P.27469-009, $48 \mathrm{~mm}$ SL, Escape Reef, QLD; P.28554-003, 3 specimens, 44-59 $\mathrm{mm}$ SL, Chagos Archipelago, Indian, Ocean; P.30340-010, 2 specimens, $45-60 \mathrm{~mm} \mathrm{SL}$, Madang, PNG; P.30844-049, 29 mm SL,
Hibernia Reef, WA, Australia; P.31144-024, 2 specimens, 33-49 mm SL, Kimbe, Bay, PNG; P.31202-002, 2 specimens, 34-50 mm SL, East Montalivet Island, WA, Australia; P.31437-037, 2 specimens, $52-58 \mathrm{~mm} \mathrm{SL}$, Ashmore Reef, WA, Australia; P.31438-083, 3 specimens, 30$54 \mathrm{~mm}$ SL, Ashmore Reef, WA, Australia; P.31654-002, 2 specimens, 51-51 mm SL, Ingram Shoals, WA, Australia.

Diancistrus altidorsalis Schwarzhans, Møller and Nielsen, 2005:-95, figures_13, 14

HoLotyPe. P.30410-001, 66 mm SL, Bodgaya Islands, Malaysia.

PARATYPES. P.31213-007, $63 \mathrm{~mm}$ SL, Madang Lagoon, PNG; P.31355-026, 3 specimens, 61-79 mm SL, Irai Island, PNG; P.31491-001, 4 specimens, 38-95 $\mathrm{mm}$ SL, Togean Islands, Indonesia; P.31736-004, 35 mm SL, Panarairai, PNG.

Diancistrus beateae Schwarzhans, Møller and Nielsen, 2005: 100, figures 18, 19.

PaRATYPes. P.30618-031, 2 specimens, 53-82 mm SL, Madang, PNG; P.31144-025, 2 specimens, 3974 mm SL, Kimbe Bay, PNG; P.31438-006, 81 mm SL, Ashmore Reef, WA, Australia.

REMARKS. The type description includes an error: the paratypes P.30618-031 were collected at Madang, PNG $\left(05^{\circ} 09^{\prime} \mathrm{N}, 145^{\circ} 50^{\prime} \mathrm{E}\right)$, not Togean Islands, Indonesia.

Diancistrus fijiensis Schwarzhans, Møller and Nielsen, 2005: 110, figures 27, 28.

Paratype. P.30791-001，43 mm SL, Dravuni Island, Fiji.

Diancistrus jeffjohnsoni Schwarzhans, Møller and Nielsen, 2005: 117, figures 34, 35.

PARATYPES. P.27967-005, 3 specimens, 43-61 mm SL, Carnarvon, WA, Australia; P.27980-064, 53 $\mathrm{mm}$ SL, Monte Bello Islands, WA, Australia; P.30920-002, 4 specimens, 41-49 mm SL, Heywood Islands, WA, Australia; P.31251-055, 4 specimens, 51-59 mm SL, Montgomery Reef, WA, Australia; P.31389-001, 37 mm SL, Beagle Bay, WA, Australia.

Diancistrus mcgroutheri Schwarzhans, Møller and Nielsen, 2005: 129, figures 45, 46.

HolotyPE. P.29627-047, 36 mm SL, Holmes Reef, QLD.

PARATYPe. P.27469-010, 43 mm SL, Escape Reef, QLD.

Diancistrus niger Schwarzhans, Møller and Nielsen, 2005: 138 , figures 53,54 .

Holotype. P.31543-005, $40 \mathrm{~mm}$ SL, Mansuar Island, West Papua, Indonesia.

Paratypes. P.31541-011, $45 \mathrm{~mm}$ SL, Batanta Island, West Papua, Indonesia; P.31543-004, 4 
specimens, 30-44 mm SL, Mansuar Island, West Papua, Indonesia.

Dinematichthys dasyrhynchus Cohen and Hutchins, 1982: 342 , figure 1 .

HoLotyPE. P.26614-010, $88 \mathrm{~mm}$ SL, Rottnest Island, WA, Australia.

PARATYPES. P.4683-001, 94 mm SL; P.25725-017, 2 specimens, $102 \mathrm{~mm} \mathrm{SL}$; P.25781-013, $99 \mathrm{~mm} \mathrm{SL}$; P.26616-009, 5 specimens, $57-116 \mathrm{~mm} \mathrm{SL;}$ P.26619-015, 2 specimens, 111-121 mm SL; P.26620-010, 2 specimens, $84-119 \mathrm{~mm} \mathrm{SL;}$ P.27146-001, 2 specimens, $57-119 \mathrm{~mm} \mathrm{SL}$, all from Rottnest Island, WA, Australia.

Diplacanthopoma kreffti Cohen and Nielsen, 2002: 12, figure 1.

HOLOTYPE. P.30586-002, $96 \mathrm{~mm}$ SL, NW Shelf, WA, Australia.

Dipulus caecus Waite, 1905: 78, Plate 11 (figure 2). HoLotYPe. P.13261-001, 152 mm SL, Fremantle, WA, Australia.

Microbrotula polyactis Anderson, 2005: 38, figure 2. Paratype. P.30811-005, 29 mm SL, Sulawesi, Indonesia.

\section{Macrouridae}

Caelorinchus thurla Iwamoto and Williams, 1999: 164, figure 23.

HoLotyPe. P.21638-001, $210 \mathrm{~mm} \mathrm{SL,} \mathrm{NW} \mathrm{Shelf,}$ WA, Australia.

Paratypes. P.21639-001 to P.21642-001, 4 specimens, 105-187 mm SL, NW Shelf, WA, Australia; P.26270-016, 3 specimens, 174-193 mm SL, NW Shelf, WA, Australia.

REMARKS. The type description includes two errors: the holotype P.21638-43 should read P.21638-001 and the paratypes P.21638-43 should read P.21639-001 to P.21642-001. Specimen P.21643-001 is non-type.

\section{Hemiramphidae}

Zenarchopterus ornithocephala Collette, 1985: 107, figure 2.

Paratype. P.27868-004, 94 mm SL, Senopi, West Papua, Indonesia.

\section{Pseudomugilidae}

Chilatherina alleni Price, 1997: 79, figures 1, 2. PaRatypes. P.31029-001, 5 specimens, $44-66 \mathrm{~mm}$ SL, Siriwo, West Papua, Indonesia.

Chilatherina axelrodi Allen, 1979: 49, figure. Holotype. P.26739-001, 81 mm SL, Pual River system, PNG.
Paratypes. P.26739-002, 22 specimens, 35-85 mm SL, Pual River system, PNG.

Chilatherina bleheri Allen, 1985b: 54, figures 1, 2 . PARATYPES. P.27869-001, 30 specimens, $24-84 \mathrm{~mm}$ SL (missing); P.27870-001, 4 specimens, 74-90 $\mathrm{mm}$ SL; P.27871-001, 10 specimens, 47-92 mm SL, all from Lake Holmes, West Papua, Indonesia.

Chilatherina pricei Allen and Renyaan, 1996a: 5, figures 1, 2, 3 .

Paratypes. P.31033-011, 4 specimens, 53-79 mm SL; P.31034-008, 7 specimens, 59-81 mm SL; P.31041-002, $68 \mathrm{~mm}$ SL, all from Reifafeif River, West Papua, Indonesia.

Glossolepis dorityi Allen, 2001a: 767, figures 1, 2. Paratypes. P.31761-001, 6 specimens, 78-115 mm SL, Lake Nenggwambu, West Papua, Indonesia. REMARKS. The type description includes an error: for the paratypes P.31761-001 there are 6 specimens, not 14 . The other eight paratypes are at MZB (MZB 10934).

Glossolepis leggetti Allen and Renyaan, 1998: 72, figures 2, 3.

Paratypes. P.31455-002, 24 specimens, $44-78 \mathrm{~mm}$ SL, Tiawiwa River tributary, West Papua, Indonesia;

P.31459-004, 93 mm SL, Wapoga River tributary, West Papua, Indonesia.

Glossolepis maculosus Allen, 1981a: 302, figure 1.

HoLotyPe. P.26977-001, 46 mm SL, Omsis River, PNG.

PaRATYPes. P.26976-003, 47 mm SL, Omsis River, PNG; P.26977-004, 3 specimens, 24-44 mm SL, Omsis River, PNG.

REMARKS. The type description includes two errors: the holotype P.26976-001 should read P.26977-001 and the paratypes P.26976-004 should read P.26977-004.

Glossolepis pseudoincisus Allen and Cross, 1980: 392 , figure 5 .

PARATYPES. P.26793-001, 5 specimens, $60-77 \mathrm{~mm}$ SL, Tami River, West Papua, Indonesia.

Glossolepis ramuensis Allen, 1985b: 56, figure 4. HoLotYPE. P.28187-004, 55 mm SL, Ramu River, PNG.

REMARKS. The type description includes an error: the type locality of Ramuy River should read Ramu River.

Glossolepis wanamensis Allen and Kailola, 1979: 40, figures 1, 2.

HoLOTYPe. P.26420-001, 80 mm SL, Lake Wanam, PNG.

Paratypes. P.26420-002, 31 specimens, 57-83 mm 
SL, Lake Wanam, PNG.

REMARKS. The type description lists 35 paratypes for P.26420-002. Four specimens were donated to AMNH (AMNH 51593) in 1982, leaving 31 specimens at WAM.

Kiunga ballochi Allen, 1983a: 73, figures 1a, 1b. Holotype. P.27797-001, 24 mm SL, Fly River tributary, PNG.

Paratypes. P.27797-002, 40 specimens ( $3 \mathrm{c} / \mathrm{s}), 15-$ $26 \mathrm{~mm} \mathrm{SL}$, Fly River tributary, PNG; P.27798001, 21 specimens, $18-28 \mathrm{~mm}$ SL, Fly River tributary, PNG.

Kiunga bleheri Allen, 2004a: 80, figures 1, 2, 3, 4. HoLOTYPE. P.32455-001, 28 mm SL, Tare Creek, PNG.

Paratypes. P.31572-001, 2 specimens, 20-25 mm SL, Fly River tributary, PNG; P.32455-002, 4 specimens, $17-26 \mathrm{~mm}$ SL, Tare Creek, PNG.

REMARKS. The 2 specimens in paratypes P.31572001 includes one specimen originally P.31572002.

Melanotaenia ajamaruensis Allen and Cross, 1980: 384 , figure 2.

PARATYPES. P.26792-001, 5 specimens, 44-68 mm SL, Ajamaru Lakes, West Papua, Indonesia.

REMARKS. The type description includes an error: for the paratypes P.26792-001 there are 5 specimens, not 6 .

Melanotaenia angfa Allen, 1990: 103, figure 3. ParatyPes. P.29969-001, 31 specimens (2 c/s), 32 $97 \mathrm{~mm}$ SL, Pondok Creek, West Papua, Indonesia; P.29971-001, 2 specimens, 105-114 mm SL, Kurumoi Creek, West Papua, Indonesia.

Melanotaenia arfakensis Allen, 1990: 105, figure 4. PARATYPes. P.29950-001, 62 specimens (4 c/s), 21$66 \mathrm{~mm}$ SL, Sub River, West Papua, Indonesia.

Melanotaenia batanta Allen and Renyaan, 1998: 75, figure 6.

Paratypes. P.31461-001, 5 specimens, 39-66 mm SL, Batanta Island, Indonesia.

Melanotaenia boesemani Allen and Cross, 1980: 379, figure 1.

PARATYPES. P.26791-001, 3 specimens, $50-63 \mathrm{~mm}$ SL, Ajamaru Lakes, West Papua, Indonesia.

Melanotaenia caerulea Allen, 1996a: 10, figures 1, 2. HoLOTYPE. P.30986-001, $62 \mathrm{~mm}$ SL, tributary of $\mathrm{Au}$ Creek, PNG.

Paratypes. P.30966-001, 23 specimens, 24-47 mm SL, Kikori, PNG; P.30980-001, 26 specimens, 24$40 \mathrm{~mm} \mathrm{SL}$, Kikori, PNG; P.30981-005, 6 specimens, 22-32 mm SL, Au Creek, PNG; P.30984-002, 47 specimens, 22-57 mm SL, Mubi River, PNG; P.30985-002, 4 specimens, 30-36 mm SL, Ofake Creek, PNG; P.30986-002, 6 specimens, 38-52 mm SL, Au Creek, PNG.

Melanotaenia corona Allen, 1982a: 174, figure 1.

ParatyPes. P.27386-001, $85 \mathrm{~mm}$ SL, Sermowai River, PNG.

Melanotaenia eachamensis Allen and Cross, 1982: 45.

Paratypes. P.26386-001, 76 specimens, 25-63 mm SL, Lake Eacham, Qld., Australia

REMARKS. The type description lists 80

specimens for paratypes P. 26386-001. However, four specimens were donated to AMNH (AMNH 51596) in 1982, leaving 76 specimens at WAM.

Melanotaenia exquisita Allen, 1978: 97, figure.

Holotype. P.25897-001, 59 mm SL, Edith River, NT, Australia.

PARATYPes. P.25897-002, 96 specimens (5 c/s), 20 $49 \mathrm{~mm}$ SL, Edith River, NT, Australia.

REMARKs. The type description lists 105 paratypes for P.25873-002. Five specimens were donated to AMNH (AMNH 51595) in 1982, leaving 96 specimens at WAM. The location of the remaining four is unknown.

Melanotaenia gracilis Allen, 1978: 98, figure.

Holotype. P.25410-011, 68 mm SL, Drysdale River, WA, Australia.

PARATYPES. P.25402-007, 4 specimens, 53-58 mm SL, Morgunda Creek, WA, Australia; P.25404005, 19 specimens $(4 \mathrm{c} / \mathrm{s}), 32-66 \mathrm{~mm} \mathrm{SL}$, Morgunda Creek, WA, Australia; P.25405-002, 4 specimens, 28-42 mm SL, Morgunda Creek, WA, Australia; P.25406-005, 3 specimens, $28-30 \mathrm{~mm}$ SL, Morgunda Creek, WA, Australia; P.25410006, 13 specimens, 32-64 mm SL, Drysdale River, WA, Australia; P.25426-004, 7 specimens, 27-43 mm SL, Forest Creek, WA, Australia; P.25427-006, 35 specimens, 27-66 mm SL, Drysdale River, WA, Australia; P.25457-004, 9 specimens $(4 \mathrm{c} / \mathrm{s}), 30-55 \mathrm{~mm}$ SL, Crossland Creek, WA, Australia; P.25873-002, 74, 21-52 mm SL, Drysdale River, WA, Australia.

REMARKS. The type description lists 80 paratypes for P.25873-002. Five specimens were donated to AMNH (AMNH 51619) in 1982, leaving 74 specimens at WAM. The location of the remaining specimen is unknown.

Melanotaenia herbertaxelrodi Allen, 1981b: 31, figure.

Holotype. P.26972-001, $61 \mathrm{~mm}$ SL, Purari River, PNG.

Paratypes. P.26972-002, 36 specimens, 26-84 mm SL, Purari River, PNG.

REMARKs. The type description lists 41 paratypes for P.26972-002. Five specimens were donated to 
AMNH (AMNH 51593) in 1982, leaving 36 specimens at WAM.

Melanotaenia irianjaya Allen, 1985b: 58, figures 7, 8 . PARATYPES. P.27863-001, 12 specimens, 29.5-50.5 mm SL, Fruata Stream, West Papua, Indonesia; P.27864-001, 32 specimens, 21-55 mm SL, Merdai River, West Papua, Indonesia; P.27867001, 41 specimens, 13-53 mm SL, Auk River, West Papua, Indonesia; P.27868-001, 17 specimens, $14-47 \mathrm{~mm}$ SL, Senopi River, West Papua, Indonesia.

REMARKS. The type description includes two errors: the paratypes P.27868-001 should read P.27867-001 and the paratypes P.27869-001 should read P.27868-001.

Melanotaenia iris Allen, 1987a: 15, figures 1, 2.

HolotyPe. P.28480-001, 95 mm SL, Logatyu River, PNG.

Paratypes. P.28480-002, 2 specimens, $79-82 \mathrm{~mm}$ SL, Logatyu River, PNG.

Melanotaenia kamaka Allen and Renyaan, 1996b: 15, figures 2, 3, 14, 15.

Paratypes. P.30519-001, 10 specimens, $42-60 \mathrm{~mm}$ SL, Triton Lakes, West Papua, Indonesia.

Melanotaenia lakamora Allen and Renyaan, 1996b: 17 , figures $6,7,8,16,17$.

PARATYPES. P.31042-004, 6 specimens, 256-54 mm SL, Triton Lakes, West Papua, Indonesia; P.31043-001, 22 specimens, 38-52 mm SL, Triton Lakes, West Papua, Indonesia.

Melanotaenia maculata Weber, 1907: 239, Plate 11 (figure 4).

Paralectotype. P.26783-001, 73 mm SL, Merauke River, PNG.

REMARKS. This specimen was transferred to WAM from ZMA in 1979 (original registration number ZMA 110-168).

Melanotaenia maylandi Allen, 1983b: 84, figures 1, 2.

Paratypes. P.27869-002, 8 specimens, $42-89 \mathrm{~mm}$ SL, Lake Holmes, West Papua, Indonesia.

Melanotaenia misoolensis Allen, 1982b: 107, figure 1.

Paratypes. P.27379-001, 2 specimens, 53-58 mm SL, Misool Island, Indonesia.

REMARKS. The type description includes an error: the paratypes P.27279-001 should read P.27379001.

Melanotaenia monticola Allen, 1980: 45, figure 5. HoLotype. P.26747-001, 68 mm SL, Omei Creek, PNG.

Paratypes. P.26747-002, 20 specimens, $21-74 \mathrm{~mm}$ SL, Omei Creek, PNG.
Remarks. The type description includes two errors: the holotype P.26741-001 should read P.26747-001 and the paratypes P.26741-002 should read P.26747-002.

Melanotaenia mubiensis Allen, 1996a: 13, figure 5. Holotype. P.30984-001, 93 mm SL, Mubi River, PNG.

Paratypes. P.30981-002, 5 specimens, 19-73 mm SL, Au Creek, PNG; P.30983-001, 8 specimens, 19-96 mm SL, Digibu River, PNG; P.30984-006, 20 specimens, 23-80 mm SL, Mubi River, PNG; P.30985-001, 2 specimens, 46-55 mm SL, Ofake Creek, PNG.

Melanotaenia papuae Allen, 1981c: 50, figures 1, 3. Holotype. P.26393-002, $60 \mathrm{~mm}$ SL, Mount Diamond, PNG.

PARATYPES. P.26393-001, 34 specimens, 18-54 mm SL, Mount Diamond, PNG; P.26394-001, 8 specimens, 37-47 mm SL, near Port Moresby, PNG; P.26395-001, 116 specimens 19-42 mm SL, Dasiama Creek, PNG; P.26396-001， 43 specimens, 22-57 mm SL, Brown River, PNG; P.26399-001, 20 specimens, 22-39 mm SL, Veimauri, PNG; P.26400-001, 21 specimens, 1642 mm SL, Port Moresby, PNG; P.26403-001, 13 specimens, 31-60 mm SL, Goldie River, PNG; P.26404-001, 36 mm SL, Brown River, PNG; P.26405-001, 21 specimens (4 c/s), 30-44 mm SL, small stream near Brown River, PNG; P.26406001, 2 specimens, 44-48 mm SL, Eilogo Creek, PNG.

Melanotaenia parkinsoni Allen, 1980: 43, figures 1, 2.

Holotype. P.26401-003, $98 \mathrm{~mm}$ SL, near Matairuka, PNG.

Paratypes. P.26401-001, 7 specimens, 29-100 mm SL, near Matairuka, PNG; P.26402-001, 9 specimens, 26-80 mm SL, Ararabu Creek, PNG; P.26786-001, 9 specimens, 45-74 mm SL, Alotau, PNG.

Melanotaenia parva Allen, 1990: 109, figures 9, 10. Paratypes. P.29970-001, 125 specimens (4 c/s), 22-53 mm SL, Danau Kurumoi, West Papua, Indonesia.

Melanotaenia pierucciae Allen and Renyaan, 1996b: 19, figures 11, 12, 18, 19.

Paratypes. P.31044-002, 5 specimens, $36-52 \mathrm{~mm}$ SL, Werfyang Creek, West Papua, Indonesia.

Melanotaenia pimaensis Allen, 1981b: 74, figures. Holotype. P.26971-001, 64 mm SL, Pima River, PNG.

Paratypes. P.26971-002, 38 specimens, $15-55 \mathrm{~mm}$ SL, Pima River, PNG.

REMARKS. The type description lists 43 paratypes 
for P.26971-002. Five specimens were donated to AMNH (AMNH 51591) in 1982 leaving 38 specimens at WAM.

Melanotaenia pygmaea Allen, 1978: 99, figure. Holotype. P.25034-003, 46 mm SL, Cascade Creek, WA, Australia.

PARATYPES. P.25034-001, 30 specimens ( $3 \mathrm{c} / \mathrm{s}), 22-$ $43 \mathrm{~mm}$ SL, Cascade Creek, WA, Australia; P.25040-001, 10 specimens, 30-45 $\mathrm{mm} \mathrm{SL,}$ Youwanjela Creek, WA, Australia.

Melanotaenia rubripinnis Allen and Renyaan, 1998: 77, figures 10,11 , cover.

Paratypes. P.31448-001, 7 specimens, 77-100 mm SL, Tiawiwa River tributary, West Papua, Indonesia; P.31450-002, 24 specimens, 25-68 mm SL, Logari River tributary, West Papua, Indonesia; P.31451-006, 76 mm SL, Logari River tributary, West Papua, Indonesia.

REMARKS. The type description includes an error: the paratypes listed with registration number "WAM" only should include the number P.31451-006.

Melanotaenia sylvatica Allen, 1997a: 38, figure 1. HolotyPE. P.31224-001, 55 mm SL, Sapoi River, PNG.

PARATYPes. P.31224-002, 25 specimens, 32-51 mm SL, Sapoi River, PNG.

Pelangia mbutaensis Allen, 1998b: 13, figures 4, 5. Paratypes. P.31300-001, 13 specimens, 44-56 mm SL, Lake Mbutu, West Papua, Indonesia.

Popondetta connieae Allen, 1981d: 44, figures 1, 2. Holotype. P.26409-003, 43 mm SL, Auga Creek, PNG.

PARATYPES. P.26409-001, 33 specimens, 18-40 mm SL, Auga Creek, PNG; P.26410-001, 12 specimens, 14-29 mm SL, Ahaemo Creek, PNG; P.26411-001, 30 specimens, 13-35, Evundo Creek, PNG; P.26412-001, 10 specimens, 13-20 mm SL, Embi Creek, PNG; P.26416-001, 34 specimens, 12-32 mm SL, Endehi Creek, PNG; P.26417-001, 22 specimens, $15-33 \mathrm{~mm} \mathrm{SL}$, Hunete Creek, PNG; P.26418-001, 51 specimens, 16-34 mm SL, Avindo Creek, PNG.

SYNONYMY. This species is now known as Pseudomugil connieae.

Pseudomugil cyanodorsalis Allen and Sarti, 1983: 48 , figure 1 .

HoLotyPE. P.27971-001, 22 mm SL, Crab Creek, WA, Australia.

PARATYPES. P.27971-002, 454 specimens, 9-22 mm SL, Crab Creek, WA, Australia.

Pseudomugil ivantsoffi Allen and Renyaan, 1999: 164, figures $2,3$.
Paratypes. P.31051-003, 2 specimens, 21-28 mm SL, Aikwa River tributary, West Papua, Indonesia; P.31052-004, 4 specimens, 18-22 mm SL, Aikwa River tributary, West Papua, Indonesia; P.31055-004, 14 specimens, 19-28 mm SL, Kopi River tributary, West Papua, Indonesia; P.31057-004, 23 specimens, 19-28 mm SL, Iweka River tributary, West Papua, Indonesia; P.31298003, 22 mm SL, Cross Levee, West Papua, Indonesia.

Pseudomugil-mellis-Allen_and Ivantsoff,_1982: 84, figures $1,2$.

PARATYPeS. P.27439-003, 28 specimens, 14-21 mm SL, Peregian Beach, Qld., Australia

Pseudomugil paludicola Allen and Moore, 1981: 106, figure 1 .

HoLOTYPE. P.26751-001, 24 mm SL, near Tureture Village, PNG.

PARATYPes. P.26751-002, 100 specimens, 13-24 mm SL, near Tureture Village, PNG.

Pseudomugil paskai Allen and Ivantsoff, 1986: 85, figure 1.

Holotype. P.28139-002, 24 mm SL, near Kiunga, PNG.

PARATYPES. P.27794-002, 58 specimens, 15-24 mm SL; P.28139-003, 29 specimens, 13-24 mm SL; P.28140-001, 18 specimens, $12-18 \mathrm{~mm} \mathrm{SL}$, all from near Kiunga, PNG.

Pseudomugil pellucidus Allen, Ivantsoff, Shepherd and Renyaan, 1998: 2, figures 1, 16, 17, 18.

Holotype. P.31055-008, $31 \mathrm{~mm}$ SL, Kopi River tributary, West Papua, Indonesia.

Paratypes. P.31055-005, 16 specimens, 20-30 mm SL, Kopi River tributary, West Papua, Indonesia; P.31055-007, 3 specimens (all c/s), Kopi River tributary, West Papua, Indonesia; P.31057-003, 3 specimens, 20-25 mm SL, Iweka River tributary, West Papua, Indonesia.

REMARKS. The type description includes an error: the paratypes P.31055-008 should read P.31055007.

Rhombatractus affinis Weber, 1907: 234, Plate 11 (figure 5).

PARALECTOTYPE. P.26784-001, 89 mm SL, Sentani Lake Basin, PNG.

REMARKS. This specimen was transferred to WAM from ZMA in 1979 (original catalogue number ZMA 103-158).

SYNONYMY. This species is now known as Melanotaenia affinis.

Rhombatractus vanheurni Weber and de Beaufort, 1922: 299.

PARALECTOTYPES. P.26785-001, 4 specimens, 80125 mm SL, near Idenburg River, PNG. 
REMARKS. This specimen was transferred to WAM from ZMA in 1979 (original registration number ZMA 103-138).

SYNONYMY. This species is now known as Melanotaenia vanheurni.

Scaturiginichthys vermeilipinnis Ivantsoff, Unmack, Saeed and Crowley, 1991: 278, figure 1.

PARATYPeS. P.30179-001, 19 specimens, $12-17 \mathrm{~mm}$ SL, Aramac, Qld., Australia

\section{Atherinidae}

Atherinosoma wallacei Prince, Ivantsoff and Potter, 1982: 67, figures 1,2 bottom.

HoLOTYPE. P.27275-001, $41 \mathrm{~mm}$ SL, Guildford, WA, Australia.

Paratypes. P.27276-001, 4 specimens, $45-47 \mathrm{~mm}$ SL, Guildford, WA, Australia; P.27277-001, 3 specimens, 42-54 mm SL, Murray River, WA, Australia; P.27278-001, 2 specimens, $43-44 \mathrm{~mm}$ SL, Canning River, WA, Australia.

Craterocephalus amniculus Crowley and Ivantsoff, 1990a: 88, figure 1.

PaRATYPES. P.29891-001, 4 specimens, 32-41 mm SL, Cockburn River, NSW, Australia.

Craterocephalus centralis Crowley and Ivantsoff, 1990a: 92, figure 3.

PARATYPES. P.29892-001, 4 specimens, $41-46 \mathrm{~mm}$ SL, Finke River, NT, Australia; P.29893-001, 2 specimens, 44-57 mm SL, Pioneer Creek, NT, Australia.

Craterocephalus cuneiceps Whitley, 1944a: 266.

Holotype. P.1998-001, $36 \mathrm{~mm}$ SL (45 mm TL), Belele Station, WA, Australia.

Paratypes. P.1999-001 to P.2005-001, 20-35 mm SL, Belele Station, WA, Australia.

REMARKs. There has been confusion about the number of types of this species. Whitley (1944a) stated that the description was based on one specimen only, the largest of nine specimens, 20-36 mm SL, for which he provided eight registration numbers, P.1998-2005 (now P.1998001 to P.2005-001). However, he did not indicate which number pertained to the type. McKay (1966a) subsequently designated the holotype as P.1998-001, and amended the catalogue entry for the remaining specimens to read "Paratypes". Hutchins and Smith (1991) believed that the latter was unwarranted as Whitley gave no indication that more than one specimen was involved in the description. However, a later paper by Whitley (1955) indicated that the type series did involve more than one specimen, so we therefore accept that P.1999-001 to P.2005001 are valid paratypes encompassing the remaining eight specimens.
Craterocephalus fistularis Crowley, Ivantsoff and Allen, 1995: 325, figure 1.

Paratype. P.30519-002 (c/s), $59 \mathrm{~mm}$ SL, Triton Lakes, West Papua, Indonesia.

Craterocephalus gloveri Crowley and Ivantsoff, 1990b: 115, figure 1 .

Paratypes. P.30033-001, 10 specimens, 20-27 mm SL, Dalhousie Springs, SA, Australia.

Craterocephalus helenae Ivantsoff, Crowley and Allen, 1987a: 177, figure 3.

HoLOTYPE. P.25456-003, $57 \mathrm{~mm}$ SL, Drysdale River, WA, Australia.

Paratypes. P.25406-009, 2 specimens, 35-53 mm SL, Morgunda Creek, WA, Australia; P.25424008, 4 specimens $(2 \mathrm{c} / \mathrm{s}), 36-58 \mathrm{~mm}$ SL, Fern Gully Creek, WA, Australia; P.25456-012, 8 specimens, 39-61 mm SL, Drysdale River, WA, Australia; P.25873-010, 8 specimens, 44-67 mm SL, Drysdale River, WA, Australia.

REMARKS. The type description includes an error: the paratypes P.25456-003 should read P.25456012.

Craterocephalus kailolae Ivantsoff, Crowley and Allen, 1987b: 332, figure 1.

Paratypes. P.27783-001, 14 specimens $(3 \mathrm{c} / \mathrm{s}), 16-$ $42 \mathrm{~mm}$ SL, Foasi Creek, PNG.

Craterocephalus lentiginosus Ivantsoff, Crowley and Allen, 1987a: 175, figure 2.

Holotype. P.25029-003, 43 mm SL, Roe River, WA, Australia.

Paratypes. P.25028-003, 6 specimens, $28-36 \mathrm{~mm}$ SL, Wyulda Creek, WA, Australia; P.25029-007, 8 specimens (2 c/s), 22-32 mm SL, Roe River, WA, Australia.

REMARKS. The type description provides the same registration number for both the holotype and one lot of paratypes. We have amended the registration number of the paratypes to P.25029007.

Craterocephalus marianae Ivantsoff, Crowley and Allen, 1987a: 179, figure 4.

Paratypes. P.28195-001, 10 specimens, 41-54 mm SL, Magela River, NT, Australia.

Craterocephalus munroi Crowley and Ivantsoff, 1988: 156, figure 1.

Paratypes. P.29673-001, 5 specimens, 28-29 mm SL, Little Bynoe River, Qld., Australia

Craterocephalus pimatuae Crowley, Ivantsoff and Allen, 1991: 35, figure 1.

Holotype. P.26971-005, 25 mm SL, Pima River, PNG.

Paratypes. P.26971-004, 4 specimens (1 c/s), 2435 mm SL, Pima River, PNG. 
REMARKS. The type description includes an error: the holotype P.26971-004 should read P.26971005 .

Kestratherina brevirostris Pavlov, Ivantsoff, Last and Crowley, 1988: 387, figure 1.

PARATYPES. P.29154-001, 4 specimens, $41-57 \mathrm{~mm}$ SL, Western Port Bay, Vic., Australia

\section{Trachichthyidae}

Optivus agastos Gomon, 2004: 174, figure $1 a$. PARATYPES. P.25739-007, $84 \mathrm{~mm}$ SL, Moreton Bay, Qld., Australia; P.28843-001, 85 mm SL, Port Hacking, NSW, Australia.

Optivus agrammus Gomon, 2004: 176, figure $1 \mathrm{~b}$. PARATYPES. P.15263-001, $55 \mathrm{~mm}$ SL, Fremantle, WA, Australia; P.26608-018, 62 mm SL, Cheyne Beach, WA, Australia.

REMARKS. The type description includes an error: the paratype P.26608-08 should read P.26608018.

Sorosichthys ananassa Whitley, 1945: 22, figure 12. HoLOTYPE. P.734-001, $52 \mathrm{~mm}$ SL, Bald Island, WA, Australia.

\section{Berycidae}

Centroberyx australis Shimizu and Hutchins, 1987: 89 , figure 1 .

HoLotype. P.27720-001, 202 mm SL, Ocean Reef, WA, Australia.

Paratypes. P.27607-002, 2 specimens, 185-209 mm SL, Ocean Reef, WA, Australia; P.27732-002, 3 specimens, 175-191 mm SL, Ocean Reef, WA, Australia; P.28238-001, $201 \mathrm{~mm} \mathrm{SL}$, Marmion, WA, Australia.

\section{Holocentridae}

Adioryx lepros Allen and Cross, 1983: 5, figure 1.

HoLotype. P.26089-001, 77 mm SL, Christmas Island, Indian Ocean.

Paratypes. P.26106-010, $62 \mathrm{~mm}$ SL, Christmas Island, Indian Ocean; P.27663-004, 58 mm SL, Rowley Shoals, WA, Australia.

REMARKS. The paratype P.27663-004 is listed in an addendum at the end of the type description.

SYNONYMY. This species is now known as Sargocentron lepros.

Myripristis earlei Randall, Allen and Robertson, 2003: 406, figures 1, $2,3$.

PARATYPES. P.32101-001, 5 specimens, 168-199 $\mathrm{mm}$ SL, Phoenix Islands, Pacific Ocean.

REMARKs. The type description includes an error: the paratype P.32101.111 should read P.32101001.

\section{Solenostomidae}

Solenostomus halimeda Orr, Fritzsche and Randall, 2002: 101, figures $1,2,3,4,5,6$, cover.

Holotype. P.27666-030, $47 \mathrm{~mm} \mathrm{SL,} \mathrm{Rowley}$ Shoals, WA, Australia.

Paratype. P.27666-052, $41 \mathrm{~mm} \mathrm{SL,} \mathrm{Rowley}$ Shoals, WA, Australia.

\section{Syngnathidae}

-Ghoeroichthys_latispinosus Dawson, 1978a: 414, figures $1,2$.

HoLOTYPe. P.25815-024, 27 mm SL, South Muiron Island, WA, Australia.

Dunckerocampus naia Allen and Kuiter, 2004: 2, figures $1,2,3,5$.

HoLotyPE. P.32334-004, 120 mm SL, Maytag Reef, Fiji.

Paratype. P.32462-001, 74 mm SL, Maratua Island, Indonesia.

Enchelyocampus brauni Dawson and Allen, 1978: 407 , figures 6,7 .

HoLOTYPe. P.25800-001, 54 mm SL, North West Cape, WA, Australia.

SYNONYMY. This species is now known as Bulbonaricus brauni.

Heraldia nocturna Paxton, 1975: 441, figures 1, 2. Paratype. P.25167-001, $61 \mathrm{~mm}$ SL, Sydney Harbour, NSW, Australia.

Hippichthys gazella Whitley, 1947: 148.

HolotyPe. P.2871-001, $78 \mathrm{~mm}$ SL, Broome, WA, Australia.

REMARKs. Whitley's original description was brief, with no mention of a type specimen. He subsequently (1948) provided an expanded description, including full details of the holotype.

SYNONYMY. This name is a junior synonym of Hippichthys penicillus (Cantor, 1849).

Hippocampus alatus Kuiter, 2001: 317, figure 25. PARATYPe. P.28003-001, $81 \mathrm{~mm}$ height, Monte Bello Islands, WA, Australia.

Hippocampus biocellatus Kuiter, 2001: 311, figure 15.

Holotrpe. P.9398-001, 95 mm height, Shark Bay, WA, Australia.

PARATYPES. P.9402-001, $68 \mathrm{~mm}$ height; P.14584001, $108 \mathrm{~mm}$ height; P.28611-005, $98 \mathrm{~mm}$ height; P.30081-002, $60 \mathrm{~mm}$ height; P.30998-001, $95 \mathrm{~mm}$ height, all from Shark Bay, WA, Australia.

Hippocampus montebelloensis Kuiter, 2001: 326, figure 36.

PARATYPE. P.29078-001, $78 \mathrm{~mm}$ height, Exmouth 
Gulf, WA, Australia.

Histiogamphelus meraculus Whitley, 1948: 271. Holotype. P.1215-001, 225 mm SL, City Beach, WA, Australia.

REMARKs. The type was originally a dried specimen, but is now in ethanol.

SYNONYMY. This species is now known as Mitotichthys meraculus.

Lissocampus affinis Whitley, 1944a: 266.

HOLOTYPE. P.1150-001, $81 \mathrm{~mm}$ SL, Rottnest Island, WA, Australia.

SYNONYMY. This name is a junior synonym of Lissocampus runa (Whitley, 1931).

Micrognathus spinirostris Dawson and Allen, 1981: 65 , figures 1,3 .

HoLOTYPE. P.26479-001, 103 mm SL, North West Cape, WA, Australia.

SYNONYMY. This species is now known as Halicampus spinirostris.

Syngnathus parvicarinatus Dawson, 1978b: 288, figures 1,2 .

HoLotyPe. P.25801-001, 79 mm SL, Darwin Port, NT, Australia.

SYNONYMY. This species is now known as Hippichthys parvicarinatus.

\section{Synbranchidae}

Anommatophasma candidum Mees, 1962a: 27.

HoLotyPE. P.4917-001, 370 mm TL, North West Cape, WA, Australia.

Paratype. P.4918-001, $316 \mathrm{~mm}$ TL, North West Cape, WA, Australia.

SYNONYMY. This species is now known as Ophisternon candidum.

\section{Scorpaenidae}

Centropogon australis latifrons Mees, 1962b: 28, Plate 1.

Holotype. P.5140-001, 76 mm SL, Cheynes Bay, WA, Australia.

Paratypes. P.4871-001, 2 specimens, 73-75 mm SL, Cheyne Bay, WA, Australia (1 specimen missing, presumed lost); P.4872-001, 54 mm SL, Geraldton, WA, Australia.

Remarks. Paxton et al. (1989) erroneously cited P.4871-001 as the holotype.

SYNONYMY. Incorrectly synonymised with $C$. australis (White, 1790) by Paxton, et al. (1989). This species is now known as Centropogon latifrons.

Cheroscorpaena tridactyla Mees, 1964a: 1, Plate 1. Paratype. P.8285-001, $85 \mathrm{~mm}$ SL, Frederick Hendrik Island, PNG.
Inimicus cirrhosus McKay, 1964: 8, figure 1.

HoLoTYPe. P.4980-001, 125 mm SL, Shark Bay, WA, Australia.

Paratypes. P.4981-001, $150 \mathrm{~mm}$ SL, Shark Bay, WA, Australia; P.4982-001, 180 mm SL, Shark Bay, WA, Australia; P.4983-001, 155 mm SL, Exmouth Gulf, WA, Australia (missing, presumed lost); P.4984-001, 203 mm SL, Shark Bay, WA, Australia; P.4985-001, $167 \mathrm{~mm} \mathrm{SL}$, Shark Bay, WA, Australia; P.4986-001, 190 mm SL, Shark Bay, WA, Australia; P.4987-001, 256 mm SL, Houtman Abrolhos, WA, Australia; P.4988-001, 56 mm SL, Shark Bay, WA, Australia (missing, presumed lost); P.4989-001, $56 \mathrm{~mm} \mathrm{SL}$, Shark Bay, WA, Australia; P.4990-001, 106 mm SL, Shark Bay, WA, Australia; P.4991-001, 90 mm SL, Shark Bay, WA, Australia; P.4992-001, 77 mm SL, Shark Bay, WA, Australia; P.4993001, $93 \mathrm{~mm}$ SL, Shark Bay, WA, Australia; P.4994-001, 119 mm SL, Shark Bay, WA, Australia; P.4995-001, 106 mm SL, Shark Bay, WA, Australia.

SYNONYMY. This name is a junior synonym of Inimicus sinensis (Valenciennes, 1833).

Maxillicosta lopholepis Eschmeyer and Poss, 1976: 441, figure 6.

HoLOTYPE. P.5736-001, 46 mm SL, Bernier Island, WA, Australia.

Paratype. P.25384-001, 39 mm SL, Bernier Island, WA, Australia.

Scorpaenodes steenei Allen, 1977a: 145, Plate 1. HolotyPE. P.25178-006, 93 mm SL, Rottnest Island, WA, Australia.

Scorpaenopsis possi Randall and Eschmeyer, 2001: 54, Plates VII (figures b, c, d), XII (figures a, b, c).

Paratypes. P.26102-037, 146 mm SL, Christmas Island, Indian Ocean; P.29055-011, 2 specimens, 115-125 mm SL, Ashmore Reef, WA, Australia. REMARKS. The type description includes an error: the paratypes P.262102-037 should read P.26102037.

Scorpaenopsis ramaraoi Randall and Eschmeyer, 2001: 64, Plates VIII (Figs b, c, d), XII (figure d).

Paratypes. P.31778-001, 5 specimens, $91-135 \mathrm{~mm}$ SL, Halmahera Island, Indonesia.

\section{Neosebastidae}

Neosebastes longirostris Motomura, 2004: 41, Plate $1 \mathrm{~g}$; figures 2c, 3e, 4b, 6b, 7a, 8d, 9d, 19.

Paratypes. P.26193-016, 2 specimens, 81-82 mm SL, Monte Bello Islands, WA, Australia; P.26262003, $148 \mathrm{~mm}$ SL, Dampier Land, WA, Australia; P.28264-005, $133 \mathrm{~mm}$ SL, Scott Reef, WA, Australia. 
Neosebastes occidentalis Motomura, 2004: 51, Plate IIb; figures 2c, 3h, 4b, 6b, 7a, 8f, 9f, 11b, 22 .

Holotype. P.22272-002, 145 mm SL, Cape Cuvier, WA, Australia.

PARATyPes. P.22272-001, 2 specimens, 141-148 mm SL, Cape Cuvier, WA, Australia; P.22391001, 3 specimens, 149-154 mm SL, Cape Cuvier, WA, Australia; P.23903-001, 2 specimens, 123$131 \mathrm{~mm}$ SL, North West Cape, WA, Australia; P.26182-007, 4 specimens, 118-141 mm SL, -Muiren-Islands, WA, Australia; P.31122-002, 3 specimens, $156-180 \mathrm{~mm}$ SL, Point Cloates, WA, Australia.

Scorpaena gasta Motomura, Last and Yearsley, 2006: 361 , figures $1,2,3$.

HolotyPe. P.27960-006, 69 mm SL, Kalbarri, WA, Australia.

PARATYPes. P.26069-002, $84 \mathrm{~mm}$ SL, Shark Bay, WA, Australia; P.32317-005, 74 mm SL, Shark Bay, WA, Australia.

Scorpaena panda Richardson, 1842: 216.

NEOTYPE. P.22132-001, $207 \mathrm{~mm}$ SL, Cockburn Sound, WA, Australia.

REMARKS. The orginal type description was based on a drawing and the location of the specimen (from Houtman Abrolhos, WA, Australia) is unknown. Motomura (2004) designated a Cockburn Sound specimen as a neotype to clarify taxonomic issues.

SYNONYMY. This species is now known as Neosebastes pandus.

\section{Triglidae}

Pterygotrigla draiggoch Richards, Yato and Last, 2003: 5, Plate 1 (figures 4, 5).

PARATYPES. P.26182-012, $92 \mathrm{~mm}$ SL, Muiron Islands, WA, Australia; 26187-006, $208 \mathrm{~mm}$ SL, Monte Bello Island, WA, Australia; P.26193-019, 4 specimens, 73-91 mm SL, NNW Rosemary Island, WA, Australia.

REMARKS. The type description includes several errors: the locality Muirow Island should read Muiron Islands; the paratype P.26182 should read P.26182-012; the paratype P.26187 should read P.26187-006 and the paratypes P.26193 should read P.26193-019.

\section{Aploactinidae}

Cocotropus larvatus Poss and Allen, 1987: 79, figure 1.

Paratype. P.29008-001, 22 mm SL, Christmas Island, Indian Ocean.

Neoaploactis tridorsalis Eschmeyer and Allen, 1978: 444, figure 1.

Holotype. P.25529-001, $34 \mathrm{~mm}$ SL, One Tree

\section{Island, Qld., Australia}

Paraploactis intonsa Poss and Eschmeyer, 1978: 414, figures 2, 9 .

Holotype. P.12100-001, 125 mm SL, Shark Bay, WA, Australia.

PARATYPes. P.4714-001, 112 mm SL, Shark Bay, WA, Australia; P.5874-001, 121 mm SL, Shark Bay, WA, Australia; P.6069-001, $111 \mathrm{~mm} \mathrm{SL}$, Shark Bay, WA, Australia; P.8923-001, 126 mm SL, Shark Bay, WA, Australia; P.14683-001, 98 mm-SL,Shark-Bay,W-A,_Australia;-P.23605-001, 114 mm SL, Carnarvon, WA, Australia; P.25840001, $128 \mathrm{~mm}$ SL, Shark Bay, WA, Australia.

Paraploactis pulvinus Poss and Eschmeyer, 1978: 413, figures 2, 8 .

Holotype. P.25528-001, $105 \mathrm{~mm}$ SL, Exmouth Gulf, WA, Australia

Paratypes. P.20123-001, $119 \mathrm{~mm}$ SL, Rosemary Island, WA, Australia; P.23735-001, 93 mm SL, Exmouth Gulf, WA, Australia.

\section{Platycephalidae}

Cociella hutchinsi Knapp, 1996: 30, figure 9.

PARATYPES. P.30716-001, 4 specimens, 142-184 mm SL, Timor Sea, WA, Australia.

Onigocia bimaculata Knapp, Imamura and Sakashita, 2000: 3, figures 1, 2, 3 .

PARATypes. P.29637-038, 2 specimens, 24-27 mm SL, Georgina Cay, Qld., Australia

Platycephalus chauliodous Knapp, 1991: 24, figures 1,3 .

Paratypes. P.24172-001, $453 \mathrm{~mm}$ SL, Carnac Island, WA, Australia; P.25798-008, 66 mm SL, Eagle Bay, WA, Australia; P.29879-001, 332 mm SL, off Perth, WA, Australia; P.29944-001, 499 mm SL, Cowaramup Beach, WA, Australia.

Rogadius patriciae Knapp, 1987: 54.

PARATyPe. P.26212-004, $179 \mathrm{~mm}$ SL, North West Shelf, WA, Australia.

Sunagocia sainsburyi Knapp and Imamura, 2004: 547, figures 1 .

Holotype. P.26230-007, 86 mm SL, Port Hedland, WA, Australia.

\section{Congiopodidae}

Congiopodus coriaceus Paulin and Moreland, 1979: 604, figures 2, 3 .

Paratype. P.26435-001, 176 mm SL, Campbell Island, New Zealand.

\section{Chandidae}

Parambassis altipinnis Allen, 1982c: 166, figure 1. 
Paratype. P.27378-001, 7 specimens, 74-87 mm SL, Idenburg River, West Papua, Indonesia.

\section{Acropomatidae}

Malakichthys levis Yamanoue and Matsuura, 2001: 1140 , figures $1,2 \mathrm{a}$.

PARATYPES. P.26795-001, 2 specimens, 140-141 $\mathrm{mm}$ SL, Cape Leveque, WA, Australia.

\section{Serranidae}

Acanthistius pardalotus Hutchins, 1981: 493, figures $1 \mathrm{~b}, 2$.

HoLotYPe. P.5113-001, 189 mm SL, Kalbarri, WA, Australia.

Paratypes. P.3784-001, $236 \mathrm{~mm}$ SL, City Beach, WA, Australia; P.4465-001, $184 \mathrm{~mm}$ SL, Mullaloo Beach, WA, Australia; P.4502-001, 183 mm SL, Rottnest Island, WA, Australia; P.4791-001, 197 mm SL, Rottnest Island, WA, Australia; P.4792001, 248 mm SL, Port Gregory, WA, Australia; P.5577-001, $62 \mathrm{~mm}$ SL, Port Gregory, WA, Australia; P.5777-001, $275 \mathrm{~mm}$ SL, Maragret River, WA, Australia; P.14532-001, 370 mm SL, Houtman Abrolhos, WA, Australia; P.14533-001, $163 \mathrm{~mm}$ SL, Point Quobba, WA, Australia; P.16775-001, 265 mm SL, Margaret River, WA, Australia; P.26664-004, 277 mm SL, Shark Bay, WA, Australia; P.26825-001, 2 specimens, 94-118 mm SL, Rottnest Island, WA, Australia.

Anthias engelhardi Allen and Starck, 1982: 48, figures 2, 3 .

PARATYPE. P.27483-001, 66 mm SL, Great Barrier Reef, Qld., Australia

SYNONYMY. This species is now known as Pseudanthias engelhardi.

Anthias georgei Allen, 1976a: 28, figure 2.

HoLOTYPE. P.25205-001, $79 \mathrm{~mm}$ SL, Bernier Island, WA, Australia.

Paratypes. P.25205-002, 2 specimens, 29-33 mm SL, Bernier Island, WA, Australia.

SYNONYMY. This species is now known as Pseudanthias georgei.

Anthias hutomoi Allen and Burhanuddin, 1976: 46, figure 1.

PARATYPes. P.25199-001, 4 specimens, $28-49 \mathrm{~mm}$ SL, Ceram, Indonesia.

SYNONYMY. This species is now known as Pseudanthias hutomoi.

Anthias lori Lubbock and Randall in Fourmanoir and Laboute, 1976: 287, figures.

Paratype. P.25493-001, $33 \mathrm{~mm}$ SL, Tetiaroa Atoll, Society Islands.

SYNONYMY. This species is now known as Pseudanthias lori.
Anthias pictilis Randall and Allen, 1978: 34, figures $1,2$.

PaRaTYPeS. P.25939-001, 3 specimens, 70-91 mm SL, North Rock, Lord Howe Island, NSW, Australia.

SYNONYMY. This species is now known as Pseudanthias pictilis.

Anthias randalli Lubbock and Allen, 1978: 260, figures 1, 2.

Paratypes. P.25233-007, 6 specimens, $22-41 \mathrm{~mm}$ SL, Ambon Island, Indonesia; P.25239-002, 8 specimens, 35-47 mm SL, Ambon Island, Indonesia; P.25628-001, 3 specimens, $54-56 \mathrm{~mm}$ SL, Bairakaseru Island, Palau.

SYNONYMY. This species is now known as Pseudanthias randalli.

Anthias rubrizonatus Randall, 1983: 35, figures 5, 6, $7,8$.

Paratype. P.27647-001, 52 mm SL, Guadalcanal, Solomon Islands.

SYNONYMY. This species is now known as Pseudanthias rubrizonatus.

Anthias smithvanizi Randall and Lubbock, 1981: 9, figure 8 .

Paratypes. P.25492-001, 4 specimens, $27-48 \mathrm{~mm}$ $\mathrm{SL}$, Ambon Island, Indonesia.

SYNONYMY. This species is now known as Pseudanthias smithvanizi.

Caesioscorpis theagenes Whitley, 1945: 21, figure 11.

HolotyPe. P.2795-001, 140 mm SL, Carnarvon, WA, Australia.

Cephalopholis xanthopterus Allen and Starck, 1975: 246 , figure 1.

Paratype. P.25244-008, $112 \mathrm{~mm}$ SL, Ambon Island, Indonesia.

SYNONYMY. This name is a junior synonym of Cephalopholis cyanostigma (Valenciennes, 1828).

Ellerkeldia rubra Allen, 1976a: 24, figure 1.

Holorype. P.25314-003, 71 mm SL, Houtman Abrolhos, WA, Australia.

Paratypes. P.25226-001, $73 \mathrm{~mm}$ SL, Cape Inscription, WA, Australia; P.25311-007, 2 specimens, $28-50 \mathrm{~mm}$ SL, Houtman Abrolhos, WA, Australia.

SYNONYMY. This name was shown to be invalid by Allen and Randall (1990) (preoccupied by Scopularia rubra De Buen, 1959). They proposed the new name Hypoplectrodes cardinalis noting that Hypoplectrodes is the senior synonym of Ellerkeldia (Anderson and Heemstra, 1989).

Ellerkeldia wilsoni Allen and Moyer, 1980: 329, figures $1,2$. 
HoLotype. P.26531-010, $151 \mathrm{~mm}$ SL, Geographe Bay, WA, Australia.

Paratypes. P.26531-011, 2 specimens, 77-133 mm SL, Geographe Bay, WA, Australia; P.26532-001, 5 specimens, 59-144 mm SL, Geographe Bay, WA, Australia; P.26533-001, 150 mm SL, Rottnest Island, WA, Australia.

SYNONYMY. This species is now known as Hypoplectrodes wilsoni.

Epinephelus bilobatus Randall and Allen, 1987: 406, figure 4.

HolotyPe. P.25119-008, 156 mm SL, Dampier Archipelago, WA, Australia.

PARATYPES. P.14924-001, $101 \mathrm{~mm}$ SL, Yampi Sound, WA, Australia; P.14948-001, 80 mm SL, Port Walcott, WA, Australia; P.24358-001, 238 mm SL, Dampier Archipelago, WA, Australia.

Epinephelus homosinensis Whitley, 1944a: 267.

HoLotyPE. P.2413-001, 235 mm SL, Broome, WA, Australia.

Paratypes. P.2412-001, $251 \mathrm{~mm}$ TL, Broome, WA, Australia; P.2430-001, $128 \mathrm{~mm}$ TL, Houtman Abrolhos, WA, Australia.

REMARKS. Whitley's description gives the type locality as Geraldton, WA, Australia, but WAM catalogue entries made in 1942 record Broome as the collection site for the holotype and first paratype, and the Houtman Abrolhos (off Geraldton) as the collection site for the second paratype. Therefore, we consider the type locality to be Broome.

SYNONYMY. This name is a junior synonym of Epinephelus rivulatus (Valenciennes, 1830).

Epinephelus rankini Whitley, 1945: 24.

HoLOTYPE. P.2847-001, 330 mm SL, Onslow, WA, Australia.

SYNONYMY. This name is a junior synonym of Epinephelus multinotatus (Peters, 1876).

Epinephelus spiramen Whitley, 1945: 25.

HoLOTYPE. P.2796-001, 263 mm SL, Carnarvon, WA, Australia.

SYNONYMY. This name is a junior synonym of Epinephelus rivulatus (Valenciennes, 1830).

Epinephelus stictus Randall and Allen, 1987: 394, figure 1 .

HoLotype. P.28277-001, $328 \mathrm{~mm}$ SL, Heywood Shoals, WA, Australia.

Epinephelus timorensis Randall and Allen, 1987: 399 , figure 2.

HoLotype. P.24794-001, $141 \mathrm{~mm}$ SL, Dillon Shoals, WA, Australia.

Epinephelus trophis Randall and Allen, 1987: 402, figure 3.
Holotype. P.24795-001, $105 \mathrm{~mm}$ SL, Dillon Shoals, WA, Australia.

Lepidoperca aurantia Roberts, 1989: 580, figure 8. PaRATYPes. P.28301-001, 2 specimens, 193-269 mm SL, Westport, New Zealand.

Lepidoperca filamenta Roberts, 1987: 83.

PARATYPES. P.26804-001, 2 specimens, 107-127 mm SL, Eucla, WA, Australia.

Liopropoma multilineatum Randall and Taylor, 1988: 41, Plate 2c.

Paratypes. P.28024-007, $61 \mathrm{~mm}$ SL, Rowley Shoals, WA, Australia; P.28186-017, 46 mm SL, Kavieng, PNG.

Liopropoma tonstrinum Randall and Taylor, 1988: 38 , Plate $2 b$.

Paratypes. P.26082-015, 28 mm SL; P.26087-011, 3 specimens, 28-65 mm SL; P.26121-002, $54 \mathrm{~mm}$ SL, all from Christmas Island, Indian Ocean.

Plectranthias alleni Randall, 1980: 113, figure 2.

Holotype. P.25625-001, $52 \mathrm{~mm}$ SL, WA, Australia.

Paratypes. P.23309-001, $55 \mathrm{~mm}$ SL, Lancelin, WA, Australia; P.23322-001, 17 mm SL, Rottnest Island, WA, Australia; P.23323-001, 23 mm SL, Dongara, WA, Australia; P.23325-001, 10 specimens, 30-59 mm SL, Green Head, WA, Australia; P.23334-001, 62 mm SL, Rottnest Island, WA, Australia.

REMARKS. The type description includes the registration number P.23325-30 for ten specimens from Geen Head originally accessioned under six separate numbers (P.23325-001 to P.23330-001). They have been amalgamated as P.23325-001.

Plectranthias fourmanoiri Randall, 1980: 125, figure 7.

PARATypes. P.26085-029, 28 mm SL; P.26113-004, 2 specimens, $16-20 \mathrm{~mm}$ SL; P.26126-005, $21 \mathrm{~mm}$ $\mathrm{SL}$, all from Christmas Island, Indian Ocean.

Plectranthias inermis Randall, 1980: 135, figure 11. Paratypes. P.26121-001, 2 specimens, $19-20 \mathrm{~mm}$ SL, Christmas Island, Indian Ocean; P.26125-008, $26 \mathrm{~mm}$ SL, Christmas Island, Indian Ocean.

Plectranthias nanus Randall, 1980: 159, figure 22. Paratypes. P.26090-002, $32 \mathrm{~mm}$ SL, Christmas Island, Indian Ocean; P.26122-003, 2 specimens, 25-26 mm SL, Christmas Island, Indian Ocean.

Pseudanthias sheni Randall and Allen, 1989: 74, figures $1,2,3,4,5$.

HolotYPE. P.29660-001, 96 mm SL, Rowley Shoals, WA, Australia. 
Paratype. P.27668-001, 85 mm SL, Rowley Shoals, WA, Australia.

Rabaulichthys altipinnis Allen, 1984a: 48, figure 1. HoLOTYPE. P.28179-001, 45 mm SL, Rabaul, PNG. Paratypes. P.28180-001, 3 specimens, $36-45 \mathrm{~mm}$ SL, Rabaul, PNG.

Sacura parva Heemstra and Randall, 1979: 9, figure 2; Plate 4.

HoLOTYPE. P.24785-001, 67 mm SL, Dillon Shoals, WA, Australia.

Paratype. P.26459-001, 17 mm SL, Dillon Shoals, WA, Australia.

Serranus socorroensis Allen and Robertson, 1992a: 38, figure 1.

PARATYPes. P.30390-001, 2 specimens, 37-41 mm SL, Socorro Island, Mexico.

\section{Nannopercidae}

Nannoperca variegata Kuiter and Allen, 1986: 111, figures 1, 2, 3, 4 .

PaRATypes. P.28597-001, 9 specimens, 37-50 mm SL, Winnap, Vic., Australia; P.28598-001, $47 \mathrm{~mm}$ SL, Winnap, Vic., Australia

\section{Pseudochromidae}

Assiculoides desmonotus Gill and Hutchins, 1997: 45 , figures $5,6,7$.

HoLotyPe. P.31085-009, 47 mm SL, Long Island, Vansittart Bay, WA, Australia.

Paratypes. P.30321-021, 9 specimens, 25-49 mm SL, Sunday Island, WA, Australia; P.30898-008, 7 specimens, 34-47 mm SL, Sunday Island, WA, Australia; P.30908-003, 36 mm SL, Mermaid Island, WA, Australia; P.30910-009, 6 specimens, 34.4-47.2 mm SL, Bedford Islands, WA, Australia; P.30916-008, 45 mm SL, Admiral Island, WA, Australia; P.30929-008, 10 specimens (2 c/s), 11-48 mm SL, Whirlpool Pass, WA, Australia; P.31078-006, 6 specimens, 22-51 $\mathrm{mm}$ SL, Jones Island, WA, Australia; P.31084003, 2 specimens, 26-41 mm SL, Vansittart Bay, WA, Australia; P.31085-037, 5 specimens, 34-51 mm SL, Vansittart Bay, WA, Australia; P.31097021, 2 specimens, $44-51 \mathrm{~mm} \mathrm{SL}$, Cape Londonderry, WA, Australia.

Congrogadus winterbottomi Gill, Mooi and Hutchins, 2000: 74, figures 2, 5.

Holotype. P.31582-001, $85 \mathrm{~mm}$ SL, Middle Mangrove Island, WA, Australia.

Paratypes. P.31013-046, 2 specimens, 66-112 mm SL, Exmouth Gulf, WA, Australia; P.31017-022, 3 specimens, 72-116 mm SL, Exmouth Gulf, WA, Australia; P.31018-012, 4 specimens, 67-119 mm SL, Exmouth Gulf, WA, Australia; P.31582-002, 2 specimens, $69-80 \mathrm{~mm}$ SL, Middle Mangrove Island, WA, Australia.

Manonichthys alleni Gill, 2004: 34, figures 14, 15; Plates 2d, e.

HoLotyPe. P.30415-004, 52 mm SL, Mantabuan Island, Indonesia.

PARATYPe. P.30406-009, 28 mm SL, Sibuan Island, Indonesia.

Pictichromis coralensis Gill, 2004: 59, figure 22; Plate 4 a.

Paratype. P.29638-006, 36 mm SL, Lihou Reef, Qld., Australia

Pseudochromis alticaudex Gill, 2004: 76, figures 29, 30.

Paratypes. P.24915-002, 2 specimens, $30-39 \mathrm{~mm}$ SL, Goodenough Island, PNG; P.29595-016, 29 $\mathrm{mm}$ SL, Madang, PNG.

Pseudochromis aurulentus Gill and Randall, 1998: 18, figure 1.

Paratypes. P.30957-001, 2 specimens, 43-55 mm SL, Komodo Island, Indonesia.

Pseudochromis howsoni Allen, 1995a: 83, figures 1, 2.

HolotyPe. P.30840-001, 75 mm SL, Ashmore Reef, WA, Australia.

Paratype. P.30840-004, 61 mm SL, Ashmore Reef, WA, Australia.

Pseudochromis lugubris Gill and Allen, 2004: 2, figure 1 .

Paratype. P.31355-015, 7 specimens, 46-66 mm SL, Irai Island, PNG.

Pseudochromis melanurus Gill, 2004: 143, figure 34; Plates 9f, g.'

Paratypes. P.30792-001, 2 specimens, $33-40 \mathrm{~mm}$ SL, Fiji.

Pseudochromis viridis Gill and Allen, 1996: 34, figure 1.

HolotyPE. P.29331-013, $41 \mathrm{~mm}$ SL, Christmas Island, Indian Ocean.

Paratypes. P.26120-008, $43 \mathrm{~mm}$ SL, Christmas Island, Indian Ocean; P.26122-004, 29 mm SL, Christmas Island, Indian Ocean.

ReMARKS. The type description includes an error: the paratypes P.20120-008 should read P.26120008 .

Pseudoplesiops howensis Allen, 1987b: 250, figures $1,2$.

Paratypes. P.28622-001, 2 specimens, 27-29 mm SL, Lord Howe Island, NSW, Australia.

Pseudoplesiops immaculatus Gill and Edwards, 2002: 20, figures $1,2$. 
Paratypes. P.26083-041, $31 \mathrm{~mm}$ SL, Christmas Island, Indian Ocean; P.26085-030, $26 \mathrm{~mm} \mathrm{SL,}$ Christmas Island, Indian Ocean; P.26093-015, 27 mm SL, Christmas Island, Indian Ocean; P.26104-005, $27 \mathrm{~mm}$ SL, Christmas Island, Indian Ocean (missing); P.26107-004, $24 \mathrm{~mm}$ SL, Christmas Island, Indian Ocean; P.26113-003, 2 specimens, 26-28 mm SL, Christmas Island, Indian Ocean; P.27825-034, 3 specimens, 18-25 mm SL, Manus, PNG; P.29626-001, 31 mm SL, Port Moresby, PNG (missing); P.29927-004, 24 mm SL, Direction Island, Cocos Keeling.

REMARKS. The paratypes P.26104-005 and P.29626-001 are missing, presumed lost.

Pseudoplesiops knighti Allen, 1987b: 253, figures 3, 4 . Holotype. P.28534-001, 29 mm SL, Great Barrier Reef, Qld., Australia

PARATYPes. P.25322-004, 25 mm SL, Great Barrier Reef, Qld., Australia; P.27826-067, 5 specimens, 23-25 mm SL, Manus Island, PNG; P.28531-004, 3 specimens, 15-27 mm SL, Great Barrier Reef, Qld., Australia; P.28533-013, 3 specimens, 26-30 mm SL, Great Barrier Reef, Qld., Australia; P.28534-002, 3 specimens, 27-28 mm SL, Great Barrier Reef, Qld., Australia; P.28535-015, 2 specimens, 29-32 mm SL, Great Barrier Reef, Qld., Australia

SYNONYMY. This species is now known as Amsichthys knighti.

Pseudoplesiops multisquamatus Allen, 1987b: 257, figures 5, 6 .

Holotype. P.27470-002, 42 mm SL, Escape Reef, Qld., Australia

PARATYPes. P.26082-019, $47 \mathrm{~mm}$ SL, Christmas Island, Indian Ocean; P.26087-012, 2 specimens, 36-43 mm SL, Christmas Island, Indian Ocean; P.26097-010, $38 \mathrm{~mm}$ SL, Christmas Island, Indian Ocean; P.26099-011, 2 specimens, 24-39 mm SL, Christmas Island, Indian Ocean; P.26116-010, 24 $\mathrm{mm}$ SL, Christmas Island, Indian. Ocean; P.26121-003, 29 mm SL, Christmas Island, Indian Ocean; P.27476-001, $39 \mathrm{~mm}$ SL, Escape Reef, Qld., Australia; P.27659-025, 32 mm SL, Rowley Shoals, WA, Australia; P.27826-041, 3 specimens, 31-38 mm SL, Los Negros Island, PNG; P.28170010, 3 specimens, 33-40 mm SL, Rabaul, PNG; P.28172-007, 4 specimens, 24-42 mm SL, Rabaul, PNG; P.28174-028, 39 mm SL, Rabaul, PNG; P.28541-030, 3 specimens, 16-37 mm SL, Holmes Reef, Qld., Australia

SYNONYMY. This species is now known as Lubbockichthys multisquamatus.

\section{Pseudogrammatidae}

Pseudogramma axelrodi Allen and Robertson, 1995a: 73, figure 1.
Holotype. P.30995-001, 61 mm SL, Clipperton Island, Pacific Ocean.

Paratype. P.30995-002, 5 specimens, 13-53 mm SL, Clipperton Island, Pacific Ocean.

\section{Plesiopidae}

Assessor flavissimus Allen and Kuiter, 1976: 211, figure 5 .

Holotype. P.25322-001, $39 \mathrm{~mm}$ SL, Great Barrier Reef, Cairns, Qld., Australia

PARATYPES. P-25323=001,-8-speeimens, 26-38-mm SL, Lizard Island, Qld., Australia

Assessor randalli Allen and Kuiter, 1976: 208, figure 4.

PARATyPes. P.25469-001, 3 specimens, $37-40 \mathrm{~mm}$ SL, Ishigaki Island, Japan.

REMARKS. The type description includes an error: the paratypes P.25419-001 should read P.25469001 .

Paraplesiops alisonae Hoese and Kuiter, 1984: 11, figures $1,3$.

PARATYPES. P.27136-005, 3 specimens, $72-107 \mathrm{~mm}$ SL, Victor Harbour, SA, Australia.

REMARKs. The type description includes an error: the paratypes P.21736-005 should read P.27136005.

Paraplesiops sinclairi Hutchins, 1987a: 231, figures $1,2$.

Holotype. P.28298-008, 108 mm SL, Lucky Bay, WA, Australia.

PARATYPES. P.9257-001, $45 \mathrm{~mm}$ SL, Lancelin Island, WA, Australia; P.26006-001, 2 specimens, $122 \mathrm{~mm}$ SL, Mondrain Island, WA, Australia; P.28298-020, 112 mm SL, Lucky Bay, WA, Australia.

Plesiops gracilis Mooi and Randall, 1991: 374, Plate 1a; figures 2, 3a.

PARATYPES. P.27654-027, 2 specimens, 53-54 mm SL, Rowley Shoals, WA, Australia.

Plesiops insularis Mooi and Randall, 1991: 379, Plate $1 b$, c; figures $5 a, 7$.

PARATYPes. P.25650-003, 3 specimens, $72-90 \mathrm{~mm}$ SL, Lord Howe Island, NSW, Australia.

Steeneichthys nativitatis Allen, 1987c: 13, figure 1. Holotype. P.29016-001, 25 mm SL, Christmas Island, Indian Ocean.

Steeneichthys plesiopsus Allen and Randall, 1985a: 187, figures $1,2$.

Holotype. P.28031-018, $25 \mathrm{~mm}$ SL, Rowley Shoals, WA, Australia.

Paratype. P.28027-005, $18 \mathrm{~mm}$ SL, Rowley Shoals, WA, Australia. 
Trachinops brauni Allen, 1977b: 62, figures 1a, 2 . HoLOTYPE. P.25545-001, 42 mm SL, Rottnest Island, WA, Australia.

Paratypes. P.4894-001, 4 specimens, $32-40 \mathrm{~mm}$ SL, Rottnest Island, WA, Australia; P.25164-005, $41 \mathrm{~mm}$ SL, Rottnest Island, WA, Australia; P.25197-004, 2 specimens, 32-36 mm SL, Rottnest Island, WA, Australia; P.25251-010, 2 specimens, 43-55 mm SL, Rottnest Island, WA, Australia; P.25308-004, 4 specimens, 23-27 mm SL, Houtman Abrolhos, WA, Australia; P.25315-005, 3 specimens, 23-30 mm SL, Houtman Abrolhos, WA, Australia.

\section{Acanthoclinidae}

Beliops xanthokrossos Hardy, 1985: 378, figure 10. HoLotyPe. P.26617-008, 22 mm SL, Rottnest Island, WA, Australia.

PARATYPES. P.25765-011, $26 \mathrm{~mm}$ SL, Recherche Archipelago, WA, Australia; P.26006-013, 22 mm SL, Recherche Archipelago, WA, Australia; P.26617-021, 3 specimens, 14-24 mm SL, Rottnest Island, WA, Australia; P.27616-034, 2 specimens, 21-22 mm SL, Rottnest Island, WA, Australia; P.27953-013, 2 specimens, 21-22 mm SL, Jurien Bay, WA, Australia; P.27955-013, 22 mm SL, Port Denison, WA, Australia.

Remarks. The type description includes a paratype with the same registration number as the holotype, P.26617-008. That paratype has now been combined with paratypes P.26617-021, which had originally been reported as 2 specimens, $14-17 \mathrm{~mm}$ SL (all 3 specimens were collected with the holotype). The registration numbers are incorrectly presented in the type description with a space between the second and third digits.

\section{Terapontidae}

Hannia greenwayi Vari, 1978: 244, figure 39.

Holotype. P.25380-002, 81 mm SL, Hann River, WA, Australia (destroyed).

Paratypes. P.25380-001, 48 specimens, $47-89 \mathrm{~mm}$ SL, Hann River, WA, Australia (5 specimens destroyed).

REMARKS. The holotype and 5 paratypes were allowed to dry out while on loan and are no longer existent (Hutchins and Smith, 1991).

Hephaestus epirrhinos Vari and Hutchins, 1978: 4 figure 6.

Holotype. P.25724-001, 99 mm SL, Drysdale River, WA, Australia.

PARATYPeS. P.25403-002, $253 \mathrm{~mm}$ SL, Morgunda Creek, WA, Australia; P.25404-003, 4 specimens, 40-179 mm SL, Morgunda Creek, WA, Australia; P.25407-003, 72 mm SL, Morgunda Creek, WA,
Australia; P.25410-001, 3 specimens, 66-113 mm SL, Drysdale River, WA, Australia; P.25421-001, 2 specimens, $195-310 \mathrm{~mm}$ SL, Palmoondoora Creek, WA, Australia.

REMARKS. The type description includes an error: paratypes P.25421-001 were collected by handline, not rotenone.

Hephaestus komaensis Allen and Jebb, 1993: 235, figures 2, 3.

HoLoTYPE. P.30348-004, 143 mm SL, Koma River, PNG.

Paratypes. P.30348-005, 22 specimens, 31-110 mm SL, Koma River, PNG.

Hephaestus lineatus Allen, 1984b: 393, figures 1, 2. Paratypes. P.27867-002, 2 specimens, 22-23 mm SL, Auk River, West Papua, Indonesia; P.27868002, $42 \mathrm{~mm}$ SL, Senopi River, West Papua, Indonesia.

REMARKs. The type description includes two errors: the paratypes P.27868-002 should read P.27867-002 and the paratypes P.27869-002 should read P.27868-002.

Leiopotherapon macrolepis Vari, 1978: 235, figure 34.

Holotype. P.25392-001, 92 mm SL, near Prince Regent River, WA, Australia.

Paratypes. P.25025-002, $38 \mathrm{~mm}$ SL, Roe River, WA, Australia; P.25026-003, 5 specimens, 35-53 mm SL, Roe River, WA, Australia; P.25027-003, 6 specimens, 35-43 mm SL, Roe River, WA, Australia; P.25029-002, 20 specimens, 40-65 mm SL, Roe River, WA, Australia; P.25032-005, 10 specimens, $30-50 \mathrm{~mm} \mathrm{SL}$, Gundarara Creek, WA, Australia; P.25040-002, 12 specimens, 40116 mm SL, Youwanjela Creek, WA, Australia (3 specimens destroyed).

REMARKS. Three specimens of paratypes P.25040002 were allowed to dry out while on loan and are no longer existent (Hutchins and Smith 1991). The type description includes two errors: the paratypes P-25-25-002 should read P.25025002 and the paratypes P.25025-002, 1, $38 \mathrm{~mm}$ should read P.25032-002, 10, 30-50 mm.

Mesopristes jenkinsi Whitley, 1945: 26.

Holotype. P.2763-001, 116 mm SL, Ord River, WA, Australia.

SYNONYMY. This species is now known as Hephaestus jenkinsi.

Pingalla midgleyi Allen and Merrick, 1984: 76, figures $1,2$.

PaRATYPES. P.26702-002, $61 \mathrm{~mm}$ SL, East Alligator River, NT, Australia; P.27050-001, 5 specimens, 50-61 mm SL, Deaf Adder Creek, NT, Australia; P.27051-002, 4 specimens, 52-74 mm SL, Katherine River, NT, Australia. 
REMARKS. The type description includes an error: for the paratypes P.27050-001, there are 5 specimens, not 7 .

Scortum neili Allen, Larson and Midgley, 1993: 71, figure 1.

Paratypes. P.30285-001, 3 specimens, $78-86 \mathrm{~mm}$ SL, Angalarri River, NT, Australia.

Syncomistes butleri Vari, 1978: 311, figures 87, 88. HoLotYPE. P.25385-001, 123 mm SL, Barramundi Creek, NT, Australia (destroyed).

Paratypes. P.21536-001, $60 \mathrm{~mm}$ SL, Ord River, WA, Australia; P.25386-001, 5 specimens, 54-78 mm SL, South Alligator River, NT, Australia; P.25387-001, 9 specimens, 57-211 mm SL, Katherine River, NT, Australia; P.25388-001, 11 specimens, 55-175 mm SL, South Alligator River, NT, Australia; P.25389-001, 5 specimens, 44-57 mm SL, East Mary River, NT, Australia; P.25390-001, 8 specimens, 62-69 mm SL, West Baines River, NT, Australia; P.25427-003, 2 specimens, 74-88 mm SL, Drysdale River, WA, Australia.

REMARKS. The holotype was allowed to dry out while on loan and is no longer existent (Hutchins and Smith 1991). The type description includes an error: for the paratypes P.25427-003 there are 2 specimens, not 1 .

Syncomistes kimberleyensis Vari, 1978: 315, figure 91.

HoLOTYPE. P.25383-001, 36 mm SL, Bow River, WA, Australia (destroyed).

REMARKS. The holotype was allowed to dry out while on loan and is no longer existent (Hutchins and Smith 1991). Two paratypes are registered at AMNH.

Syncomistes rastellus Vari and Hutchins, 1978: 1, figure 1.

HoLOTYPE. P.25723-001, 100 mm SL, Gibb River, WA, Australia.

PARATYPES. P.25402-004, 3 specimens, 74-84 mm SL; P.25404-010, 3 specimens, $66-81 \mathrm{~mm} \mathrm{SL;}$ P.25405-007, $85 \mathrm{~mm}$ SL, all from Mogurnda Creek, WA, Australia; P.25410-004, 8 specimens, 72-125 mm SL, Drysdale River, WA, Australia.

Syncomistes trigonicus Vari, 1978: 316, figures 92, 93.

Holotype. P.25391-001, 93 mm SL, Roe River, WA, Australia (destroyed).

PARATYPES. P.25026-002, 2 specimens, 47-112 mm SL, Roe River, WA, Australia; P.25027-002, 130 mm SL, Roe River, WA, Australia; P.25028-006, $98 \mathrm{~mm}$ SL, Wyulda Creek, WA, Australia.

REMARKS. The holotype was allowed to dry out while on loan and is no longer existent (Hutchins and Smith 1991).
Therapon aheneus Mees, 1963: 2, figure 1.

HOLOTYPE. P.5350-001, $90 \mathrm{~mm}$ SL, Millstream Pool, WA, Australia (destroyed).

PARATYPES. P.5351-001, 11 specimens, 51-101 mm SL; P.5358-001, 3 specimens, 59-70 mm SL; P.5373-001, 5 specimens, 68-95 mm SL, all from Millstream Pool, WA, Australia; P.5423-001, 2 specimens, 47-51 mm SL, Fortescue River, WA, Australia (missing); P.5424-001, $50 \mathrm{~mm} \mathrm{SL}$, Fortescue River, WA, Australia (missing).

REMARKS. The holotype was allowed to dry out while on loan and is ne-longer existent (Hutchins and Smith 1991). In addition, paratypes P.5423-001 and P.5424-001 are missing, presumed lost.

SynONYMY. This species is now known as Leiopotherapon aheneus.

\section{Priacanthidae}

Priacanthus fitchi Starnes, 1988: 164, Plate 1h; figure $3 e$.

PARATYPes. P.25394-003, $88 \mathrm{~mm}$ SL; P.25399-012, 7 specimens, 69-185 mm SL; P.26291-007, 3 specimens, 95-105 mm SL, all from vicinity of Rowley Shoals, WA, Australia.

Priacanthus sagittarius Starnes, 1988: 178, Plate 3 e, $f$, g; figures $3,5,8,12,18$.

Paratype. P.29373-001, 175 mm SL, Bali, Indonesia.

\section{Apogonidae}

Apogon capricornis Allen and Randall, 1993: 112, figure 5.

PARATYPES. P.30429-001, 10 specimens, 54-57 mm SL, One Tree Island, Qld., Australia

SYNONYMY. This species is now known as Ostorhinchus capricornis.

Apogon caudicinctus Randall and Smith, 1988: 2, figure 1.

Paratype. P.29385-001, $53 \mathrm{~mm} \mathrm{SL}$, Marotiri, Pacific Ocean.

Apogon cladophilos Allen and Randall, 2002a: 122, figures 4,5 .

HoLotYPe. P.31480-001, 30 mm SL, Lizard Island, Qld., Australia

Apogon flavus Allen and Randall, 1993: 109, figures $2,3,4$.

Paratypes. P.30428-001, 2 specimens, 89-92 mm SL, Lord Howe Island, NSW, Australia.

Apogon franssedai Allen, Kuiter and Randall, 1994: 33, figure 5.

Paratypes. P.30511-001, $53 \mathrm{~mm}$ SL, Pasir Sari Reef, Indonesia; P.30514-001, 2 specimens, 41$43 \mathrm{~mm}$ SL, Bali, Indonesia. 
REMARKS. The type description includes an error: the paratypes P.30511 should read P.30511-001.

Apogon fuscomaculatus Allen and Morrison, 1996: 439, figure 1.

Paratype. P.30992-001, 49 mm SL, Rowley Shoals, WA, Australia.

Apogon fusovatus Allen, 1985c: 11, figure 1.

HolotyPe. P.14397-001, 94 mm SL, Darwin, NT, Australia.

Paratypes. P.14516-001, 84 mm SL, Darwin, NT, Australia; P.14517-001, 88 mm SL, Darwin, NT, Australia; P.28315-001, $66 \mathrm{~mm}$ SL, Aru Islands, Indonesia; P.28316-001, 83 mm SL, Darwin, NT, Australia.

REMARKS. In the original description, the paratypes P.14516-17 should be read as P.14516001 and P.14517-001. In addition, the data for paratypes P.28316-001 and P.28315-001 has been inadvertently transposed.

SYNONYMY. This name is a junior synonym of Apogon melanopus Weber, 1911.

Apogon komodoensis Allen, 1998c: 27, figure 1. Paratypes. P.30960-001, $57 \mathrm{~mm}$ SL, Rinca Island, Indonesia; P.30963-001, 2 specimens, $53-54 \mathrm{~mm}$ SL, Padar Island, Indonesia.

Apogon leptofasciatus Allen, 2001b: 145, figure 1. Paratypes. P.31782-001, 2 specimens, $48-50 \mathrm{~mm}$ SL, Wruwarez Island, Indonesia.

Apogon limenus Randall and Hoese, 1988: 361, figures 1, 2.

PARATYPes. P.27082-006, 5 specimens, $81-96 \mathrm{~mm}$ SL, Northwest Solitary Island, NSW, Australia.

REMARKS. The type description includes an error: the locality for these paratypes was incorrectly given as Solitary Island.

Apogon lineomaculatus Allen and Randall, 2002a: 117, figures 1, 2.

Paratypes. P.30514-002, 2 specimens, $45-47 \mathrm{~mm}$ SL, Bali, Indonesia.

Apogon nanus Allen, Kuiter and Randall, 1994: 29, figures 2,3 .

Paratypes. P.30372-002, 34 specimens, $13-27 \mathrm{~mm}$ SL, Madang, PNG; P.30402-002, 85 specimens, 16-29 mm SL, Mamutik Island, Indonesia.

Apogon neotes Allen, Kuiter and Randall, 1994: 31, figure 4.

Paratypes. P.30354-001, 17 specimens, $13-27 \mathrm{~mm}$ SL, Madang, PNG.

Apogon ocellicaudus Allen, Kuiter and Randall, 1994: 28, figure 1.

HolotyPe. P.29049-025, 49 mm SL, Ashmore Reef, WA, Australia.
Paratypes. P.29049-003, 7 specimens, $30-46 \mathrm{~mm}$ SL, Ashmore Reef, WA, Australia; P.30513-001, 13 specimens, $35-47 \mathrm{~mm}$ SL, Flores, Indonesia.

Apogon oxygrammus Allen, 2001b: 147, figure 4. Paratypes. P.31780-001, 2 specimens, 31-33 mm $\mathrm{SL}$, Pef Island, Indonesia.

Apogon pallidofasciatus Allen, 1987d: 3, figure 1. HolotyPE. P.28985-001, 100 mm SL, North West Cape, WA, Australia.

PARATYPES. P.25114-004, $79 \mathrm{~mm}$ SL, Dampier Archipelago, WA, Australia; P.25117-009, 86 mm SL, Dampier Archipelago, WA, Australia; P.27274-046, 5 specimens, 74-80 $\mathrm{mm} \mathrm{SL,}$ Broome, WA, Australia.

Apogon (Jaydia) photogaster Gon and Allen, 1998: 3 , figures 1 .

HolotyPe. P.31213-057, 49 mm SL, Madang Lagoon, PNG.

Paratypes. P.30358-001, $30 \mathrm{~mm}$ SL, Madang, PNG; P.31213-001, 3 specimens, 31-49 mm SL, Madang, PNG; P.31394-001, 2 specimens, 66-72 $\mathrm{mm}$ SL, Galoc Island, Philippines.

SYNONYMY. This name appears to be a junior synonym of Apogon argyrogaster Weber, 1909 (see Fraser, 2000).

Apogon pselion Randall, Fraser and Lachner, 1990: 57 , figures 8,9 .

Paratypes. P.29386-001, 2 specimens, $25-35 \mathrm{~mm}$ SL, El Himeira, Egypt.

Apogon sabahensis Allen and Kuiter, 1994: 19, figure 1.

HoLOTYPE. P.30399-012, 100 mm SL, Gaya Island, Indonesia.

Paratypes. P.30399-001, 4 specimens, 89-92 mm SL, Gaya Island, Indonesia (all missing).

SYNONYMY. This name is a junior synonym of Apogon urostigma (Bleeker, 1874).

Apogon selas Randall and Hayashi, 1990: 399, figures 1, 2.

Paratype. P.29863-001, $24 \mathrm{~mm}$ SL, Iriomote Islands, Japan.

SyNONYMY. This species is now known as Ostorhinchus selas.

Apogon smithvanizi Allen and Randall, 1994: 24, figure 1 .

HoLotype. P.25988-001, 49 mm SL, Bahrain, Arabian Gulf.

Paratypes. P.25784-004, 2 specimens, 44-47 mm SL, Ghukra, Oman.

SYNONYMY. This name is a junior synonym of Apogon gularis Fraser and Lachner, 1984.

Apogon (Jaydia) striatodes Gon, 1997: 179, figure 15. 
Paratype. P.31189-001, $45 \mathrm{~mm}$ SL, Luzon, Philippines.

Apogon unitaeniatus Allen, 1995b: 177, figure 1.

Paratype. P.30743-001, $48 \mathrm{~mm}$ SL, Bonaparte Archipelago, WA, Australia.

Apogon ventrifasciatus Allen, Kuiter and Randall, 1994: 35, figure 7 .

Holotype. P.30351-001, $49 \mathrm{~mm}$ SL, Madang, PNG.

PARATYPES. P.25244=011, 47-mm-SL, Ambon Island, Indonesia; P.30351-002, $40 \mathrm{~mm} \mathrm{SL,}$ Madang, PNG.

SYNONYMY. This name is a junior synonym of Apogon moluccensis Valenciennes, 1832.

Apogon virgulatus Allen and Randall, 1993: 107, figure 1.

Holotype. P.30312-021, $38 \mathrm{~mm}$ SL, Cleghorn Island, WA, Australia.

PARATYPes. P.30312-015, 17 specimens, 22-39 mm SL, Cleghorn Island, WA, Australia; P.30402-001, 5 specimens, $22-48 \mathrm{~mm} \mathrm{SL}$, Mamutik Island, Indonesia.

SYNONYMY. This name is a junior synonym of Apogon cavitiensis (Jordan and Seale, 1907).

Cercamia cladara Randall and Smith, 1988: 7, figure 2.

Paratype. P.29655-001, 27 mm SL, Rapa, Pacific Ocean.

Cheilodipterus alleni Gon, 1993: 10, Plate 4 (figure a); figure 4 .

HoLotype. P.30364-006, $77 \mathrm{~mm}$ SL, Madang, PNG.

PARATYPes. P.30340-002, $64 \mathrm{~mm}$ SL, Madang, PNG; P.30361-001, 2 specimens, 77-104 mm SL, Madang, PNG.

Cheilodipterus intermedius Gon, 1993: 19, Plate 1 (figure b); figure 8.

PARATYPES. P.27824-072, 2 specimens, 53-65 mm SL, Manus Island, PNG.

Cheilodipterus parazonatus Gon, 1993: 37, Plate 5 (figure c); figure 15.

PARATYPES. P.24717-001, 2 specimens, 53-61 mm SL, Lizard Island, Qld., Australia

Cheilodipterus persicus Gon, 1993: 41, Plates 3 (a), 5 (d); figure 16.

Paratypes. P.25979-001, 3 specimens, 89-96 mm SL, Persian Gulf, Qatar; P.26463-005, 84 mm SL, Persian Gulf, Qatar.

Fowleria flammea Allen, 1993a: 10, figure 3.

HoLOTYPE. P.30376-004, 28 mm SL, Madang, PNG.

Paratypes. P.27827-022, 3 specimens, $30-31 \mathrm{~mm}$ SL, Manus Island, PNG.
Glossamia timika Allen, Hortle and Renyaan, 2000: 169 , figures.

PARATYPES. P.31253-007, 8 specimens, $25-68 \mathrm{~mm}$ SL, Kopi River, West Papua, Indonesia.

Paxton concilians Baldwin and Johnson, 1999: 1051, figures 1, 2.

PARATYPE. P.27219-035, $67 \mathrm{~mm}$ SL, Houtman Abrolhos, WA, Australia.

REMARKS. The type description includes an error: the paratype P.27219035 should read P.27219035 .

Pseudamia hayashii Randall, Lachner and Fraser, 1985: 11, figure 4.

Paratypes. P.27827-023, $30 \mathrm{~mm}$ SL, Manus Island, PNG; P.28031-023, $38 \mathrm{~mm}$ SL, Rowley Shoals, WA, Australia; P.28174-004, 2 specimens, 35-54 mm SL, Rabaul, PNG.

Pseudamia nigra Allen, 1992a: 697, figure 1. HolotyPe. P.30300-001, 50 mm SL, King George River, WA, Australia.

Pseudamia zonata Randall, Lachner and Fraser, 1985: 17, Plate 1 (figure d); figures 5, 6.

PARATYPES. P.27493-001, 2 specimens, 14-42 mm SL, Rabaul, PNG.

Quinca mirifica Mees, 1966: 83, figure 1.

HolotYPe. P.5787-001, $80 \mathrm{~mm}$ SL, Yampi Sound, WA, Australia.

Rhabdamia eremia Allen, 1987d: 4, figure 2.

HoLOTYPE. P.25815-020, 37 mm SL, South Muiron Island, WA, Australia.

PARATYPes. P.25793-003, 6 specimens, 25-31 mm SL, Jiddah, Saudi Arabia; P.26071-013, $26 \mathrm{~mm}$ SL, Houtman Abrolhos, WA, Australia; P.26085015, $26 \mathrm{~mm} \mathrm{SL}$, Christmas Island, Indian Ocean; P.26092-023, 2 specimens, 20-21 mm SL, Christmas Island, Indian Ocean; P.27459-006, 30 mm SL, Escape Reef, Qld., Australia; P.27581001, $40 \mathrm{~mm}$ SL, Houtman Abrolhos, WA, Australia; P.28024-009, 22 mm SL, Rowley Shoals, WA, Australia.

SYNONYMY. This species is now known as Cercamia eremia.

Rhabdamia spilota Allen and Kuiter, 1994: 21, figure 2.

Paratypes. P.30515-001, 5 specimens, $20-34 \mathrm{~mm}$ SL, Bali, Indonesia.

Siphamia corallicola Allen, 1993a: 12, figures 4, 5, 6. HolotYPe. P.30337-009, 29 mm SL, Madang, PNG.

Paratypes. P.30337-002, 7 specimens, $19-30 \mathrm{~mm}$ SL, Madang, PNG; P.30372-004, 8 specimens, 1727 mm SL, Madang, PNG; P.30375-002, 24 specimens, $10-22 \mathrm{~mm} \mathrm{SL}$, Madang, PNG; 
P.30402-005, 5 specimens, $17-20 \mathrm{~mm} \mathrm{SL}$, Mamutik Island, Indonesia; P.30405-014, 30 mm SL, Mamutik Island, Indonesia.

Siphamia jebbi Allen, 1993a: 14, figure 7.

HoLoTYPE. P.30375-006, 22 mm SL, Madang, PNG.

Paratypes - P.27666-013, 2 specimens, 14-15 mm SL, Rowley Shoals, WA, Australia; P.30337-006, 5 specimens, 17-24 mm SL; P.30372-006, $14 \mathrm{~mm}$ SL; P.30375-001, 20 specimens, 12-22 mm SL; P.30376-019, $25 \mathrm{~mm}$ SL, all from Madang, PNG.

Vincentia badia Allen, 1987d: 6, figure 3.

HoLotyPe. P.25999-006, 68 mm SL, Cape Peron, WA, Australia.

Paratype. P.26060-006, $43 \mathrm{~mm}$ SL, Rottnest Island, WA, Australia; P.26532-007, $58 \mathrm{~mm} \mathrm{SL}$, Geographe Bay, WA, Australia; P.27643-009, 2 specimens, 47-61 mm SL, Bunkers Bay, WA, Australia; P.27956-009, 2 specimens, 39-40 mm SL, Port Denison, WA, Australia; P.28967-001, 39 mm SL, Garden Island, WA, Australia.

REMARKS. The type description includes an error: the type locality was incorrectly given as Port Peron, instead of Point Peron, but in fact should have been Cape Peron (see ICSM, 2005).

Vincentia macrocauda Allen, 1987d: 7, figure 4. Holotype. P.26608-017, $70 \mathrm{~mm}$ SL, Cheyne Beach, WA, Australia.

Paratypes. P.28293-023, $53 \mathrm{~mm}$ SL, Lucky Bay, WA, Australia; P.28297-015, 2 specimens, 40-43 mm SL, Lucky Bay, WA, Australia; P.28298-010, $84 \mathrm{~mm}$ SL, Lucky Bay, WA, Australia; P.28864005, $62 \mathrm{~mm} \mathrm{SL}$, Recherche Archipelago, WA, Australia.

\section{Sillaginidae}

Sillago (Parasillago) attenuata McKay, 1985: 36, figures 12a, 13c.

Paratype. P.28581-001, 2 specimens, 121-146 mm SL, Ras Tannurah, Saudi Arabia.

REMARKS. These paratypes were originally described with the registration number PO.491 (see History section in Introduction). A third, cleared and stained specimen (102 $\mathrm{mm}$ SL) with number PO.491 in the jar lead to confusion in Hutchins and Smith (1991). The type status of this specimen is unknown. Since lengths were not provided in the type description, specimens have been measured and reported here.

Sillago (Parasillago) bassensis flindersi McKay, 1985: 29, figures $9 \mathrm{i}, \mathrm{j}, \mathrm{k}, 1,14 \mathrm{k}$.

Paratypes. P.19116-001, $171 \mathrm{~mm}$ SL, Lakes Entrance, Vic., Australia; P.28583-001, 20 specimens (all c/s), 159-188 mm SL, Moreton Bay, Qld., Australia; P.28588-001 (c/s), 206 mm
SL, Lakes Entrance, Vic., Australia; P.28608-001 (c/s), Lakes Entrance, Vic., Australia

REMARKS. In the type description, several paratypes were originally described using a $\mathrm{PO}$ registration number (see History section in Introduction). They have been reregistered as follows: P.28583-001 (ex PO.366 to PO.385); P.28588-001 (ex PO.793) and P.28608-001 (PO.794). Paratypes PO.795 to PO.797 are missing, presumed lost. The cleared and stained P.28608-001 has disintergrated and is unmeasurable. Since lengths were not provided in the type description, specimens have been measured and reported here.

SyNONYMY. This species is now known as Sillago flindersi.

Sillago fraseri Whitley, 1944a: 270.

HOLOTYPE. P.2698-001, 93 mm SL, Bunbury, WA, Australia.

Paratype. P.2699-001, 68 mm SL, Bunbury, WA, Australia.

REMARKs. Registration numbers were omitted from the type description.

SYNONYMY. This name is a junior synonym of Sillago schomburgkii Peters, 1864.

Sillago (Parasillago) lutea McKay, 1985: 40, figures 10d, 13h, i.

HoLOTYPE. P.15928-001, $130 \mathrm{~mm}$ SL, Exmouth Gulf, WA, Australia.

Paratypes. P.13223-001 (c/s), $133 \mathrm{~mm}$ SL, Gulf of Carpentaria, Qld., Australia; P.14261-001 (missing, presumed lost); P.14398-001, $112 \mathrm{~mm}$ SL; P.14480-001 (c/s), 142 mm SL; P.14481-001 (c/ s), $124 \mathrm{~mm} \mathrm{SL} ;$ P.14482-001 (c/s), $106 \mathrm{~mm} \mathrm{SL;}$ P.14483-001 (c/s), 105 mm SL; P.15093-001 (skel.), $117 \mathrm{~mm} \mathrm{SL}$; P.15094-001 (c/s), $118 \mathrm{~mm} \mathrm{SL;}$ P.15095-001, $110 \mathrm{~mm} \mathrm{SL;} \mathrm{P.15096-001} \mathrm{(c/s),} 119$ mm SL; P.15097-001, 116 mm SL; P.15098-001, $134 \mathrm{~mm}$ SL; P.15099-001, 118 mm SL; P.15100001, $122 \mathrm{~mm}$ SL; P.15101-001, $114 \mathrm{~mm}$ SL; P.15102-001 (c/s), $122 \mathrm{~mm}$ SL; P.15103-001, 119 mm SL; P.15104-001 (c/s), 126 mm SL; P.15105001, $108 \mathrm{~mm} \mathrm{SL;} \mathrm{P.15106-001，103} \mathrm{mm} \mathrm{SL,} \mathrm{all}$ from Darwin, NT, Australia; P.15878-001, 134 mm SL; P.15879-001, 135 mm SL; P.15880-001, 128 mm SL; P.15881-001, 135 mm SL; P.15882001; P.15883-001; P.15884-001; P.15885-001， 137 mm SL; P.15886-001, 126 mm SL; P.15887-001, 125 mm SL; P.15888-001, 130 mm SL; P.15889001, $130 \mathrm{~mm}$ SL; P.15890-001, $131 \mathrm{~mm} \mathrm{SL;}$ P.15891-001, $125 \mathrm{~mm}$ SL; P.15892-001, $118 \mathrm{~mm}$ SL; P.15893-001, 130 mm SL; P.15894-001, 133 mm SL; P.15895-001, 134 mm SL; P.15896-001, 127 mm SL; P.15897-001, 128 mm SL; P.15898001, 123 mm SL; P.15899-001, 129 mm SL; P.15900-001, $130 \mathrm{~mm}$ SL; P.15901-001, 126 mm SL; P.15902-001, $143 \mathrm{~mm}$ SL; P.15903-001, 135 mm SL; P.15904-001, 131 mm SL; P.15905-001, 
128 mm SL; P.15906-001, 129 mm SL; P.15907001, $125 \mathrm{~mm} \mathrm{SL;} \mathrm{P.15908-001,} 133 \mathrm{~mm} \mathrm{SL;}$ P.15909-001, $134 \mathrm{~mm}$ SL; P.15910-001, 134 mm SL, all fromExmouth Gulf, WA, Australia; P.28582-001, 4 specimens (all c/s), 80-152 mm SL, Nickol Bay, WA, Australia; P.28584-001, 4 specimens (all c/s), 93-142 mm SL, Admiralty Gulf, WA, Australia; P.28585-001, 2 specimens (all c/s), 108-155 mm SL, Admiralty Gulf, WA, Australia; P.28586-001, 6 specimens (all c/s), 120$144 \mathrm{~mm}$ SL, Mitchell-River-area, WA, Australia; P.28589-001, 25 specimens (all c/s), 111-143 mm SL, Exmouth Gulf, WA, Australia; P.28590-001, 5 specimens (all c/s), 114-135 mm SL, Exmouth Gulf, WA, Australia; P.28602-001, 43 specimens (all skel., no skulls), 87-102 $\mathrm{mm}$ vertebral column, Admiralty Gulf, WA, Australia; P.28603-001, 7 specimens (all skel.), 105-120 mm SL, Admiralty Gulf, WA, Australia; P.28604-001, 56 specimens (all skel.), 116-135 $\mathrm{mm} \mathrm{SL,}$ Exmouth Gulf, WA, Australia; P.28605-001, 92 specimens (all skel., no skulls), 84-105 mm vertebral column, Exmouth Gulf, WA, Australia; P.28606-001, 53 specimens (all skel., no skulls), 86-103 mm vertebral column, Exmouth Gulf, WA, Australia; P.29440-002 (c/s), 116 mm SL, West Moore Island, WA, Australia.; P.29445-001 (c/s), $123 \mathrm{~mm}$ SL, King Bay, WA, Australia; P.30189-001, 13 specimens (all c/s), 93-142 mm SL, Exmouth Gulf, WA, Australia; P.30190-001, 10 specimens, Mitchell River area, WA, Australia (missing).

REMARKS. In the type description, many paratypes were originally described using a $\mathrm{PO}$ registration number (see History section in Introduction). They have been reregistered as follows: P.28589-001 (ex PO.335 to PO.359); P.28590-001 (ex PO.361 to PO.365); P.28604-001 (ex PO.386 to PO.442); P.28582-001 (ex PO.465, PO.470, PO.471); P.28605-001 (ex PO.492 to PO.583); P.28584-001 (ex PO.601 to PO.604); P.30189-001 (ex PO.605 to PO.617); P.28603-001 (ex PO.676 to PO.682); P.28606-001 (ex PO.683 to PO.735); P.28585-001 (ex PO.752 to PO.753); P.29445-001 (ex PO.762); P.28586-001 (ex PO.787 to PO.792); P.28602-001 (ex PO.822 to PO.864); P.29440-002 (ex PO.469); P.30189-001 (ex PO.605 to PO.617); P.30190-001 (ex PO.960 to PO.969). Paratypes P.28584-001 from Admiralty Gulf and paratypes P.30189-001 from Exmouth Gulf have accidentally been combined and are indistinguishable from one another. Paratypes P.15882-001 to P.15884-001 have been donated to CSIRO (C.4924 -C.4926). Since lengths for paratypes were not provided in the type description, specimens have been measured and reported here.

Sillago vincenti McKay, 1980: 378, figure 1.
Paratype. P.26850-001, $170 \mathrm{~mm}$ SL, Quilon, India.

REMARKS. Registration numbers were omitted from the type description but later provided by. McKay (1985).

Sillago (Parasillago) vittata McKay, 1985: 20, figures $4 \mathrm{~b}, 10 \mathrm{c}, 13 \mathrm{~m}, \mathrm{n}, \mathrm{o}, 141$.

Holotype. P.14172-001, 209 mm SL, Rottnest Island, WA, Australia.

PARATyPes. P.14158-001, 177 mm SL; P.14159-001, 197 mm-SL; P.14160-001,210 mm SL; P.14161001, $228 \mathrm{~mm} \mathrm{SL;} \mathrm{P.14162-001,} 172 \mathrm{~mm} \mathrm{SL;}$ P.14163-001, $202 \mathrm{~mm}$ SL; P.14164-001, 199 mm SL; P.14165-001, $160 \mathrm{~mm}$ SL; P.14166-001, 187 mm SL; P.14167-001, 227 mm SL; P.14168-001, $171 \mathrm{~mm}$ SL; P.14169-001, 239 mm SL; P.14170001, $205 \mathrm{~mm} \mathrm{SL;} \mathrm{P.14171-001,} 197 \mathrm{~mm} \mathrm{SL;}$ P.14173-001, 198 mm SL; P.14174-001 (missing); P.14175-001, $166 \mathrm{~mm}$ SL, all from Rottnest Island, WA, Australia; P.14983-001, 166 mm SL; P.15242-001, $156 \mathrm{~mm}$ SL; P.15243-001, $171 \mathrm{~mm}$ SL; P.15244-001, $171 \mathrm{~mm}$ SL; P.15245-001, 167 mm SL; P.15246-001, $169 \mathrm{~mm}$ SL, all from Shark Bay, WA, Australia; P.19230-001, 176 mm SL, Maud Landing, WA, Australia; P.28607-001, 15 specimens (all skel.), 183-202 mm SL, Shark Bay, WA, Australia.

REMARKS. The type description lists paratypes P.14158-71. This should now be read as P.14158001 to P.14171-001. In the type description, several paratypes were originally described using a PO registration number (see History section in Introduction). They have been reregistered as follows: P.28607-001 (ex PO.190 to PO.204). Since lengths were not provided in the type description, specimens have been measured and reported here.

\section{Malacanthidae}

Branchiostegus australiensis Dooley and Kailola, 1988: 253, figures $2 \mathrm{~h}, 3$.

Holotype. P.27217-004, 266 mm SL, Shark Bay, WA, Australia.

Branchiostegus gloerfelti Dooley and Kailola, 1988: 255 , figure $2 \mathrm{k}$.

HoLOTYPE. P.28304-001, 235 mm SL, Kepulauan Butu, Indonesia.

\section{Carangidae}

Caranx humerosus McCulloch, 1915: 137, Plate 25. Paratype. P.258-001, 2 specimens, 123-128 mm SL, Pine Peak Island, Qld., Australia

REMARKS. Unlike many of McCulloch's other species descriptions in the "Endeavour" series, this account does not provide details of the type series. Mention is made of the morphological 
variation amongst a series of 48 specimens, one of which was selected as the holotype. Presumably all 48 specimens were used in the description; four of those subsequently deposited at AMS have been designated as paratypes (Paxton et al., 1989). Therefore, we also recognise the two WAM specimens as paratypes. The "Endeavour" collection number of these specimens is E.2821.

SynONYMY. This species is now known as Carangoides humerosus.

Pseudocaranx dinjerra Smith-Vaniz and Jelks, 2006: 98, figures $2,3 \mathrm{~A}$.

Paratype. P.22338-001, 217 mm SL, Cape Cuvier, WA, Australia.

\section{Lutjanidae}

Lutjanus mizenkoi Allen and Talbot, 1985: 58, Plate $7 \mathrm{~h}$; figure 7.

Paratype. P.28314-001, 224 mm SL, Apolima Strait, Western Samoa.

\section{Caesionidae}

Caesio varilineata Carpenter, 1987: 24, Plates 2c, 6 g. Paratypes. P.25980-001, 96 mm SL, Persian Gulf, Qatar; P.25986-002, 276 mm SL, Persian Gulf, Bahrain; P.25997-001, 4 specimens, 167-199 mm SL, Gulf of Oman, United Arab Emirates.

Pterocaesio flavifasciata Allen and Erdmann, 2006c: 28 , figures $1,2$.

PARATYPES. P.32769-001, $203 \mathrm{~mm}$ SL, Weh Island, Indonesia; P.32770-001, 185 mm SL, Weh Island, Indonesia.

\section{Nemipteridae}

Parascolopsis rufomaculatus Russell, 1986: 137, figure 2.

HoLOTYPE. P.28105-001, $153 \mathrm{~mm}$ SL, North West Shelf, WA, Australia.

Paratypes. P.25394-010, 102 mm SL; P.26270-003, 5 specimens, $115-121 \mathrm{~mm}$ SL; P.28106-001, 146 $\mathrm{mm}$ SL, all from North West Shelf, WA, Australia.

Parascolopsis tanyactis Russell, 1986: 140, figure 3. HolotyPe. P.26263-001, $147 \mathrm{~mm}$ SL, North West Shelf, WA, Australia.

Paratypes. P.26187-003, 166 mm SL, Monte Bello Islands, WA, Australia; P.26212-001, 2 specimens, 186-188 mm SL, off Port Hedland, WA, Australia; P.26888-001, 190 mm SL, Rowley Shoals, WA, Australia; P.26889-001, 206 mm SL, Carnarvon, WA, Australia.

REMARKs. The type description includes an error: for the paratypes P.26212-001 there are 2 specimens, not 12 .
Pentapodus aureofasciatus Russell, 2001: 53, figures $1,2$.

Paratype. P.28095-001, $142 \mathrm{~mm}$ SL, Tutuila Island, American Samoa.

\section{Lethrinidae}

Lethrinus ravus Carpenter and Randall, 2003: 3, figure $1 b$.

Paratypes. P.32329-001, 2 specimens, 213-214 mm SL, Davies Reef, Qld., Australia

\section{Sciaenidae}

Nibea microgenys Sasaki, 1992: 5, figure 5.

PARATYPe. P.13689-001, 216 mm SL, Broome, WA, Australia.

\section{Mullidae}

Upeneichthys stotti Hutchins, 1990: 484, figure 1. HolotYPE. P.28939-003, 117 mm SL, Rottnest Island, WA, Australia.

PARATYPES. P.5504-001, $138 \mathrm{~mm}$ SL, Houtman Abrolhos, WA, Australia; P.5594-001, $102 \mathrm{~mm}$ SL, Bluff Rocks, WA, Australia; P.5783-001, 102 mm SL, Shark Bay, WA, Australia; P.20883-001, $112 \mathrm{~mm}$ SL, Rottnest Island, WA, Australia; P.20890-001, 110 mm SL, Rottnest Island, WA, Australia; P.22067-001, 2 specimens, 126-132 mm SL, Rottnest Island, WA, Australia; P.27219026, $98 \mathrm{~mm}$ SL, Houtman Abrolhos, WA, Australia; P.27960-011, 2 specimens, 85-99 mm SL, Kalbarri, WA, Australia; P.28614-001, 11 specimens (1 c/s), 93-115 mm SL, King George Sound, WA, Australia; P.28939-002, 5 specimens, 90-121 mm SL, Rottnest Island, WA, Australia.

\section{Monodactylidae}

Bramichthys woodwardi Waite, 1905: 72, Plate 14. HoLotype. P.13257-001, 146 mm SL (172 mm TL), Mandurah, WA, Australia.

SYNONYMY. This species is now known as Schuettea woodwardi.

\section{Pempherididae}

Pempheris elongatus McCulloch, 1911: 47, Plate 4 (figure 1).

Paratype. P.28591-001, $73 \mathrm{~mm}$ SL (90 mm TL), Flinders Island, Tas., Australia

REMARKS. This paratype was first registered as a 'co-type' in the WAM Zoological Register in 1911 as 11519. This specimen is obviously one of 16 on which the type description was based. The "Endeavour" collection number of these specimens is E.2821. The correct registration number is cited above. 
Synonymy. This species is now known as Parapriacanthus elongatus.

Pempheris ornata Mooi and Jubb, 1996: 120, figures $1,2,3,5$.

PARATYPes. P.30996-001, 2 specimens, 53-66 mm SL, Rapid Bay, SA, Australia.

Pempheris ypsilychnus Mooi and Jubb, 1996: 123, figures 7,8 .

HoLOTYPE. P.28059-019, 74 mm SL, Broome, WA,

Australia.

PARATYPES. P.28059-023, $67 \mathrm{~mm}$ SL, Broome, WA, Australia; P.28416-019, 91 mm SL, Broome, WA, Australia.

\section{Toxotidae}

Toxotes kimberleyensis Allen, 2004b: 226, figures 1, 2.

HolotyPe. P.25039-002, 126 mm SL, Plain Creek, WA, Australia.

Paratypes. P.25039-001, 3 specimens, 98-109 mm SL, Plain Creek, WA, Australia; P.25437-001, 66 mm SL, May River, WA, Australia; P.25440-006, 15 specimens, 53-93 mm SL, Lennard River, WA, Australia.

\section{Scorpididae}

Neatypus obliquus Waite, 1905: 65, Plate 10.

SYNTYPE. P.13256-001, Houtman Abrolhos, WA, Australia (missing, presumed lost).

REMARKs. The type description refers to two specimens but does not provide any registration numbers. Both were sent to AMS from WAM in 1904 (see Waite discussion in Introduction) and one was retained by AMS (AMS I.7034). The other was apparently returned to WAM and eventually registered by McKay in 1965 as P.13256-001, but it does not appear in either of McKay's lists of type specimens at WAM (McKay 1965; 1966a). As we have been unable to locate this syntype, it is considered lost.

\section{Chaetodontidae}

Chaetodon assarius Waite, 1905: 66, Plate 11 (figure 1).

HoLOTYPE. P.13255-001, 97 mm SL (119 mm TL), Fremantle, WA, Australia.

Chelmonops curiosus Kuiter, 1986a: 74, figures 1, $4 \mathrm{~b}, 5,7$.

HoLotype. P.13745-001, $137 \mathrm{~mm} \mathrm{SL,} \mathrm{Perth,} \mathrm{WA,}$ Australia.

Paratypes. P.4342-001, $179 \mathrm{~mm}$ SL, Rottnest Island, WA, Australia; P.5322-001, $121 \mathrm{~mm} \mathrm{SL}$, Beagle Bay, WA, Australia; P.24175-001, $102 \mathrm{~mm}$ SL, Wanneroo, WA, Australia; P.25725-002, 78 mm SL, Rottnest Island, WA, Australia; P.27960012, 116 mm SL, Kalbarri, WA, Australia.

Roa australis Kuiter, 2004: 168, figure 1.

Holotype. P.26215-001, $112 \mathrm{~mm}$ SL, Port Hedland, WA, Australia.

\section{Pomacanthidae}

Chaetodontoplus vanderloosi Allen and Steene, 2004: 24, figures $1,2,3,4$.

HeterTyR. P.32263=001, $125 \mathrm{~mm}$ SL, Samarai Island, PNG.

Holacanthus personifer McCulloch, 1914b: 221, Plate 31.

HolotyPe. P.28592-001, 290 mm TL, Shark Bay, WA, Australia.

SYNONYMY. This species is now known as Chaetodontoplus personifer.

\section{Pentacerotidae}

Parazanclistius hutchinsi Hardy, 1983a: 375, figure 1.

Holotype. P.25718-001, $188 \mathrm{~mm}$ SL, Rottnest Island, WA, Australia.

PARATYPES. P.725-001, $204 \mathrm{~mm} \mathrm{SL}$, Bald Island, WA, Australia; P.16411-001, 278 mm SL, Bunbury, WA, Australia.

Paristiopterus gallipavo Whitley, 1944b: 28, figure 4.

HoLotyPe. P.2589-001, 630 mm SL, Rockingham, WA, Australia.

\section{Pomacentridae}

Abudefduf lorenzi Hensley and Allen, 1977: 109, figures $1,2$.

Paratype. P.24924-001, 87 mm SL, New Britain, PNG.

Abudefduf natalensis Hensley and Randall, 1983: 2, figures 1, 2, 3a.

PARATYPes. P.26529-001, 2 specimens, 130-132 mm SL, Kwazulu, South Africa.

Altrichthys curatus Allen, 1999a: 24, figures 1, 2.

PARATYPES. P.31400-001, 6 specimens, $37-40 \mathrm{~mm}$ SL, Tangat Island, Philippines; P.31406-001, 3 specimens, $39-41 \mathrm{~mm} \mathrm{SL}$, Cuyo Islands, Philippines; P.31407-001, 8 specimens, $30-43 \mathrm{~mm}$ SL, Cuyo Islands, Philippines.

Amblyglyphidodon batunai Allen, 1995c: 86, figure 1.

Paratypes. P.25248-004, 66 mm SL, Pulau Putri, Indonesia; P.30812-002, $64 \mathrm{~mm}$ SL, Bunaken Island, Indonesia; P.30152-001, 3 specimens, 54$60 \mathrm{~mm}$ SL, Karimundajawa Island, Indonesia. 
Amblyglyphidodon flavilatus Allen and Randall, 1980: 14, figures 4, 5 .

Paratypes. P.25619-001, 2 specimens, 50-56 mm SL, Melita Bay, Red Sea.

Amblyglyphidodon indicus Allen and Randall, 2002b: 140, figures $1,2,3$.

HoLotype. P.26514-008, $93 \mathrm{~mm} \mathrm{SL}$, Villingili Island, Maldives.

PARATYPES. P.25523-008, $87 \mathrm{~mm}$ SL, Eilat, Israel; P.25527-001, 2 specimens, 70-86 mm SL, Eilat, Israel; P.26514-006, 4 specimens, $65-91 \mathrm{~mm} \mathrm{SL}$, Villingili Island, Maldives.

REMARKS. The type description includes an error: holotype P.26514-007 should read P.26514-008.

Amblypomacentrus clarus Allen and Adrim, 2000: 51 , figure 1.

PARATYPes. P.31497-002, 3 specimens, $26-34 \mathrm{~mm}$ SL, Banggai Island, Indonesia.

Amphiprion omanensis Allen and Mee, in Allen, 1991a: 220, figure (p.47).

Paratypes. P.30108-001, $64 \mathrm{~mm}$ SL, Barr Al Hikman, Oman; P.30109-001, 2 specimens, 86-93 mm SL, Barr Al Hikman, Oman; P.30110-001, 45 mm SL, Sudah, Oman.

Amphiprion rubrocinctus Richardson, 1842: 391. NEOTYPE. P.4474-001, 69 mm SL, Broome, WA, Australia.

REMARKS. Allen (1972) designated a neotype for this species as Richardson's description was based on a drawing which has subsequently been lost.

Amphiprion thiellei Burgess, 1981: 69, figures.

Paratype. P.30191-001, $39 \mathrm{~mm}$ SL, Cebu, Philippines (missing, presumed lost).

REMARKS. No registration number was provided in the type description, so the number cited above was assigned in 1991. This paratype, although reported in the type description as being housed at WAM, could not be found and is presumed lost.

Chromis abyssicola Allen and Randall, 1985b: 242, figure 1.

Paratypes. P.28421-001, 2 specimens, 111-114 mm SL, Norfolk Island Ridge, New Zealand.

Chromis alleni Randall, Ida and Moyer, 1981: 215, Plate 1 (figure a).

Paratypes. P.25516-005, 5 specimens, $47-62 \mathrm{~mm}$ SL, Sesoko Island, Japan.

REMARKS. The type description includes an error: P.25516 should read P.25516-005.

Chromis alpha Randall, 1988a: 74, figure 1.

PaRATYPes. P.24939-001, 5 specimens, $75-80 \mathrm{~mm}$ SL, Espiritu Santo Island, New Hebrides;
P.25283-001 mm SL, $82 \mathrm{~mm}$ SL, Suva Harbour, Fiji; P.27826-025, 3 specimens, 71-75 mm SL,

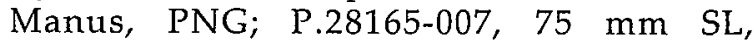
Bougainville, PNG.

REMARKS. The type description includes an error: paratypes P.27826-025 are listed twice.

Chromis caudalis Randall, 1988a: 76, figure 2.

Paratypes. P.26080-003, 75 mm SL, Christmas Island, Indian Ocean; P.26099-012, 68 mm SL, Christmas Island, Indian Ocean.

Chromis delta Randall, 1988a: 78, figure 3. Paratype. P.26099-026, $40 \mathrm{~mm}$ SL, Christmas Island, Indian Ocean.

Chromis flavaxilla Randall, 1994: 40, figures 1, 2, 3 . PaRatypes. P.25991-008, 2 specimens, $33-39 \mathrm{~mm}$ SL, Muscat, Oman; P.26461-004, 9 specimens, 34-43 mm SL, Gulf of Oman, United Arab Emirates.

Chromis flavipectoralis Randall, 1988b: 52, figures 3,4 .

Paratypes. P.26506-007, 5 specimens, $42-51 \mathrm{~mm}$ SL, Similan Islands, Thailand; P.26514-002, 55 $\mathrm{mm}$ SL, Villingili Island, Maldives.

Chromis megalopsis Allen, 1976b: 136, figure 1. HoLOTYPE. P.25207-001, 84 mm SL, Bernier Island, WA, Australia.

Paratype. P.22343-001, 102 mm SL, Cape Cuvier, WA, Australia.

REMARKS. The type description includes an error: paratype P.2234 should read P.22343-001.

Chromis monochroma Allen and Randall, 2004: 18, figure 1.

Paratype. P.32461-001, $51 \mathrm{~mm}$ SL, Flores, Indonesia.

Chromis nigroanalis Randall, 1988b: 49, figures 1, 2. Paratypes. P.26517-006, 3 specimens, $67-85 \mathrm{~mm}$ SL, Watamu, Kenya.

Chromis pelloura Randall and Allen, 1982: 16, figures 1, 2, 3.

Paratypes. P.25523-004, 2 specimens, $82-89 \mathrm{~mm}$ SL, Eilat, Israel.

Chromis pura Allen and Randall, 2004: 21, figures $2,3$.

Paratype. P.32460-001, 76 mm SL, Alor Islands, Indonesia.

Chromis trialpha Allen and Randall, 1980: 33, figures 18, 19.

Paratypes. P.25797-001, 2 specimens, $37-40 \mathrm{~mm}$ SL, Jeddah, Saudi Arabia.

Chromis westaustralis Allen, 1976b: 139, figure 2.

HoLOTYPE. P.25317-008, $84 \mathrm{~mm}$ SL, Houtman Abrolhos, WA, Australia. 
Paratypes. P.2789-001, 45 mm SL, Dirk Hartog Island, WA, Australia; P.24853-001, $27 \mathrm{~mm} \mathrm{SL}$, Albany, WA, Australia; P.25307-011, $71 \mathrm{~mm} \mathrm{SL}$, Houtman Abrolhos, WA, Australia; P.25308-006, 2 specimens, 51-60 mm SL, Houtman Abrolhos, WA, Australia; P.25317-009, $84 \mathrm{~mm} \mathrm{SL}$, Houtman Abrolhos, WA, Australia; P.25376-002, $50 \mathrm{~mm}$ SL, North West Cape, WA, Australia.

Chromis woodsi Bruner and Arnam, 1979: 50, figures 1,2 .

PARATYPE. P.26020-001, 69 mm SL, Mozambique Channel, Indian Ocean.

Chromis xanthopterygia Randall and McCarthy, 1988: 134, figures 1, 2, 3 .

PARATYPES. P.25786-002, 6 specimens, $60-76 \mathrm{~mm}$ SL, Muscat, Oman; P.25991-006, $62 \mathrm{~mm} \mathrm{SL,}$ Muscat, Oman; P.26460-008, 2 specimens, 51-63 $\mathrm{mm}$ SL, Gulf of Oman, United Arab Emirate; P.26461-005, $62 \mathrm{~mm} \mathrm{SL}$, Gulf of Oman, United Arab Emirate; P.28787-001, $80 \mathrm{~mm}$ SL, Jana Island, Saudi Arabia.

REMARKs. The type description includes two errors: P.25768-002 should read P.25786-002 and P.26461 should read P.26461-005.

Chromis xouthus Allen and Erdmann 2005: 90, figures $1,2$.

Paratypes. P. 32757-001, 2 specimens, $89-92 \mathrm{~mm}$ SL, Pulau Weh, Indonesia.

Chromis xutha Randall, 1988b: 54, figures 5, 6 .

Paratype. P.26517-001, 3 specimens, $40-47 \mathrm{~mm}$ SL, Watamu, Kenya.

Chrysiptera albata Allen and Bailey, 2002: 40, figures $1,2$.

HolotyPE. P.32109-001, 28 mm SL, Phoenix Islands, Pacific Ocean.

Paratypes. P.32109-002, 2 specimens, 22-25 mm SL, Phoenix Islands, Pacific Ocean.

Chrysiptera cymatilis Allen, 1999b: 99, figure 1. PARATYPE. P.31362-005, $31 \mathrm{~mm}$ SL, Naua Island, PNG.

Chrysiptera kuiteri Allen and Rajasuriya, 1995: 283, figure 1 .

PARATYPe. P.30827-001, 36 mm SL, Kandakulyia, Sri Lanka.

Chrysiptera pricei Allen and Adrim, 1992: 103, figure 1.

Paratypes. P.30391-001, 5 specimens, $23-31 \mathrm{~mm}$ SL, Yapen Island, West Papua, Indonesia.

Chrysiptera sheila Randall, 1994: 43, figures 5, 6, 7, 8.

Paratypes. P.30797-001, 2 specimens, 25-63 mm $\mathrm{SL}$, Arabian Sea, Oman.
REMARKS. The type description includes an error: P.30793-001 should read P.30797-001.

Chrysiptera sinclairi Allen, 1987e: 107, figure 1. HoLOTYPE. P.27827-056, 43 mm SL, Manus Island, PNG.

PARATYPes. P.27825-042, 5 specimens, $15-31 \mathrm{~mm}$ SL; P.27827-010, 14 specimens, $15-49 \mathrm{~mm} \mathrm{SL;}$ P.27828-002, 5 specimens, $18-32 \mathrm{~mm} \mathrm{SL}$, all from Manus Island, PNG.

Dascyllus_flavicaudus Randall and Allen, 1977: 375, figure 11.

Paratypes: P.25578-001, 2 specimens, $63-76 \mathrm{~mm}$ SL, Teavaraa Pass, Tahiti.

Glyphidodontops springeri Allen and Lubbock, 1976: 347, figure 1.

PARATYPES. P.25127-001, 3 specimens, 19-34 mm $\mathrm{SL}$, Ceram Island, Indonesia.

SynONYMY. This species is now known as Chrysiptera springeri.

Neoglyphidodon crossi Allen, 1991a: 221, figure (p.118).

Paratypes. P.25230-007, 4 specimens, $12-76 \mathrm{~mm}$ $\mathrm{SL}$, Ambon Island, Indonesia.

Neopomacentrus aquadulcis Jenkins and Allen, 2002: 379, figure 1 .

HoLotyPE. P.31636-001, 43 mm SL, Milne Bay, PNG.

Paratypes. P.31636-002, 9 specimens, $34-46 \mathrm{~mm}$ SL, Milne Bay, PNG.

Neopomacentrus miryae Dor and Allen, 1977: 183, figure 1 .

PARATYPE. P.25523-002, 45 mm SL, Eilat, Israel.

Neopomacentrus sororius Randall and Allen 2005: 77 , figures $5,6,7$.

PARATYPES. P.26504-005, $50 \mathrm{~mm}$ SL, Koh Hi, Thailand; P.26507-013, 38 mm SL, Koh Similan, Thailand; P.26509-003, 2 specimens, $56-58 \mathrm{~mm}$ SL, Colombo, Sri Lanka; P.26511-002, $57 \mathrm{~mm} \mathrm{SL}$, Trincomalee, Sri Lanka; P.26515-002, 47 mm SL, Shimoni, Kenya.

Neopomacentrus xanthurus Allen and Randall, 1980: 54, figures 36, 37.

PARATYPES. P.25618-001, 5 specimens, $24-41 \mathrm{~mm}$ SL, Harat Island, Ethiopia; P.25790-005, 2 specimens, 35-46 mm SL, Jeddah, Saudi Arabia; P.25794-003, 8 specimens, 39-47 mm SL, Jeddah, Saudi Arabia; P.25795-001, 4 specimens, 44-57 mm SL, Jeddah, Saudi Arabia.

Parma bicolor Allen and Larson, 1979: 12, figures 1, 3,4 .

Holotype. P.26045-001, $130 \mathrm{~mm}$ SL, Recherche Archipelago, WA, Australia. 
Paratypes. P.25468-001, $126 \mathrm{~mm}$ SL, near Albany, WA, Australia; P.25762-005, $131 \mathrm{~mm} \mathrm{SL,}$ Recherche Archipelago, WA, Australia; P.26045002, $115 \mathrm{~mm} \mathrm{SL}$, Recherche Archipelago, WA, Australia; P.26046-001, 128 mm SL, Recherche Archipelago, WA, Australia.

REMARKS. The type description includes an error: paratype P.26046-007 should read P.26046-001.

Parma kermadecensis Allen, 1987f: 267, figures 1, 2, 3.

Paratypes. P.28425-002, 2 specimens, 45-202 mm SL, Raoul Island, Kermadec Islands.

Parma occidentalis Allen and Hoese, 1975: 283, figures $2 \mathrm{f}, 15$.

HolotyPe. P.10883-001, 107 mm SL, Rottnest Island, WA, Australia.

PARATYPes. P.10877-001, 93 mm SL, Rottnest Island, WA, Australia; P.10882-001, 110 mm SL, Rottnest Island, WA, Australia; P.10940-001, 96 mm SL, Houtman Abrolhos, WA, Australia; P.10988-001, 42 mm SL, Carnarvon, WA, Australia; P.13743-001, 97 mm SL, Houtman Abrolhos, WA, Australia; P.24817-001, 2 specimens, 74-95 mm SL, Rottnest Island, WA, Australia.

Plectroglyphidodon randalli Allen, 1991a: 221, figure (p.135).

HoLotype. P.26519-001, 68 mm SL, Flic en Flac, Mauritius.

PARATYPES. P.26519-003, 4 specimens, 56-64 mm SL, Flic en Flac, Mauritius.

Pomacentrus adelus Allen, 1991a: 222, figure (p.136).

Holotype. P.29042-009, 57 mm SL, Ashmore Reef, WA, Australia.

ParatyPes. P.26505-002, 8 specimens, 54-64 mm SL, Phuket, Thailand; P.27658-029, 17 specimens, 20-50 mm SL, Rowley Shoals, WA, Australia; P.28194-016, $51 \mathrm{~mm}$ SL, Daru Island, PNG; P.29042-032, 15 specimens, 26-60 mm SL, Ashmore Reef, WA, Australia.

Pomacentrus alleni Burgess, 1981: 68, figure.

Paratypes. P.26506-009, 8 specimens, $40-45 \mathrm{~mm}$ SL, Similan Islands, Thailand.

Pomacentrus aquilus Allen and Randall, 1980: 68, figures 45, 46, 47.

Paratypes. P.25527-010, 3 specimens, $63-80 \mathrm{~mm}$ SL, Eilat, Israel.

Pomacentrus arabicus Allen, 1991a: 223, figure (p.139).

Holotype. P.25786-003, $92 \mathrm{~mm} \mathrm{SL}$, Muscat, Oman.

Paratypes. P.25786-005, 5 specimens, $64-79 \mathrm{~mm}$ SL, Muscat, Oman; P.25989-005, 3 specimens, 73-98 mm SL, Mutrah, Oman.
Pomacentrus arenarius Allen, 1987g: 9, figure 1.

Holotype. P.27465-001, 68 mm SL; Escape Reef, Qld., Australia

Paratypes. P.24940-001, $89 \mathrm{~mm}$ SL, Espiritu Santo Island, New Hebrides; P.25107-008, 2 specimens, 62-68 mm SL, Dampier Archipelago, WA, Australia; P.25371-004, 2 specimens, 60-75 mm SL, North West Cape, WA, Australia; P.27461004, $44 \mathrm{~mm}$ SL, Escape Reef, Qld., Australia; P.28161-002, 54 mm SL, New Ireland, PNG.

SYNONYMY. This name is a junior synonym of Pomacentrus nagasakiensis Tanaka, 1917.

Pomacentrus armillatus Allen, 1993b: 21, figures 1, 2.

HoLotyPe. P.30410-004, $54 \mathrm{~mm}$ SL, Bohaydulong Island, Indonesia.

PARATYPes. P.30409-001, 7 specimens, 29-54 mm SL, Bodgaya Island, Indonesia.

Pomacentrus atriaxillaris Allen, 2002: 46, figures 1, 2.

Holotype. P.32015-001, $64 \mathrm{~mm}$ SL, Mitsio Islands, Madagascar.

Paratypes. P.32024-001, 2 specimens, 25-36 mm SL, Andavakalovo Island, Madagascar.

Pomacentrus aurifrons Allen 2004c: 3, figures 1, 2. HolotyPe. P.32261-001, 62 mm SL, Toiyama Island, PNG.

PARATYPES. P.27827-038, 2 specimens, 22-42 mm SL, Manus, PNG; P.29742-001, 2 specimens, 4950 mm SL, Nagada Harbour, PNG; P.32261-002, 5 specimens, $45-54 \mathrm{~mm}$ SL, Toiyama Island, PNG.

Pomacentrus auriventris Allen, 1991a: 224, figure (p.140).

HoLotyPE. P.26126-006, $36 \mathrm{~mm}$ SL, Christmas Island, Indian Ocean.

Paratypes. P.28994-012, 2 specimens, 31-34 mm SL, Christmas Island, Indian Ocean.

Pomacentrus azuremaculatus Allen, 1991a: 225, figure (p.141).

PARATYPes. P.26506-004, 3 specimens, 47-69 mm SL, Similan Islands, Thailand.

Pomacentrus baenschi Allen, 1991a: 226, figure (p.141).

HolotyPE. P.26515-008, $66 \mathrm{~mm}$ SL, Shimoni, Kenya.

Pomacentrus bintanensis Allen, 1999b: 103, figures $5 b, 6$.

Paratypes. P.31384-001, 3 specimens, 62-79 mm SL, Bintan Island, Indonesia.

REMARKS. The type description includes an error: P.30956-001 should read P.31384-001.

Pomacentrus bipunctatus Allen and Randall, 2004: 77 , figures $1,2,3,4$. 
Paratypes. P.32354-001, 3 specimens, 51-55 mm SL, Truk Lagoon, Micronesia.

Pomacentrus caeruleopunctatus Allen, 2002: 49, figures 4, 5, 6 .

Holotype. P.32015-002, $73 \mathrm{~mm}$ SL, Mitsio Islands, Madagascar.

PARATYPes. P.32015-003, 3 specimens, $62-66 \mathrm{~mm}$ SL, Mitsio Islands, Madagascar.

Pomacentrus callainus Randall, 2002: 169, figures

$1,2$.

PARATYPe. P.31905-001, 58 mm SL, Suva Harbour, Fiji.

Pomacentrus colini Allen, 1991a: 227, figure (p.146). HolotyPE. P.29356-002, $64 \mathrm{~mm} \mathrm{SL}$, Motupore Island, PNG.

Paratypes. P.29356-001, 4 specimens, $45-58 \mathrm{~mm}$ SL, Motupore Island, PNG.

Pomacentrus cuneatus Allen, 1991a: 228, figure (p.146).

Paratypes. P.25232-001, 5 specimens, 24-71 mm SL, Ambon Island, Indonesia; P.25244-001, 8 specimens, 33-65 $\mathrm{mm} \mathrm{SL}$, Ambon Island, Indonesia.

Pomacentrus geminospilus Allen, 1993b: 23, figures $3,4$.

Holotype. P.30409-006, $50 \mathrm{~mm}$ SL, Bodgaya Island, Indonesia.

PARATYPes. P.30409-002, 7 specimens, 43-59 mm $\mathrm{SL}$, Bodgaya Island, Indonesia.

Pomacentrus indicus Allen, 1991a: 229, figure (p.148).

HOLOTYPe. P.26514-004, $74 \mathrm{~mm}$ SL, Villingili Island, Maldives.

PARATYPes. P.26514-007, 5 specimens, $37-65 \mathrm{~mm}$ SL, Villingili Island, Maldives; P.29361-001, 2 specimens, 67-75 mm SL, Chagos Archipelago, Indian Ocean.

Pomacentrus javanicus Allen, 1991a: 230, figure (p.148).

ParatyPes. P.25248-001, 2 specimens, $24-45 \mathrm{~mm}$ SL, Pulau Putri, Indonesia.

Pomacentrus komodoensis Allen, 1999b: 101, figures $3,4$.

PARATYPE. P.30956-001, 72 mm SL, Komodo Island, Indonesia.

Pomacentrus leptus Allen and Randall, 1980: 74, figures $48,49$.

PARATYPES. P.25786-001, 4 specimens, $39-55 \mathrm{~mm}$ SL, Muscat, Oman; P.26460-010, 34 mm SL, Khor Fakkan, United Arab Emirate; P.26461-010, 2 specimens, 32-38 mm SL, Khor Fakkan, United Arab Emirate.
Remarks. The type description includes two errors: P.25998-010 should read P.26460-010 and P.25999-010 should read P.26461-010.

Pomacentrus limosus Allen, 1992b: 691, figure 1.

Holotype. P.30317-001, 46 mm SL, Churchill Reef, WA, Australia.

ParatyPe. P.30317-002, 29 mm SL, Churchill Reef, WA, Australia.

Pomacentrus microspilus Allen and Randall, 2005a: 96, figures 1, 2, 3.

HoLOTYPE. P.32755-001, $65 \mathrm{~mm}$ SL, Viti Levu, FijiParatypes. P.32755-002, 2 specimens, 56-64 mm SL, Viti Levu, Fiji.

Pomacentrus polyspinus Allen, 1991a: 230, figure (p.155).

Paratypes. P.26504-010, 4 specimens, $47-56 \mathrm{~mm}$ SL, Phuket, Thailand.

Pomacentrus proteus Allen, 1991a: 231, figure (p.156).

HolotyPE. P.26509-011, 71 mm SL, Colombo, Sri Lanka.

Paratypes. P.26509-007, 4 specimens, 39-66 mm SL, Colombo, Sri Lanka.

Pomacentrus rodriguesensis Allen and Wright, 2003: 134, figures 1, 2, 3.

Paratypes. P.32237-001, 2 specimens, 52-64 mm $\mathrm{SL}$, Rodrigues Island, Indian Ocean.

Pomacentrus saksonoi Allen, 1995c: 88, figure 4. Paratype. P.30810-002, 33 mm SL, Pulau Putri, Indonesia.

Pomacentrus similis Allen, 1991a: 232, figure (p.157).

HolotyPe. P.26511-001, $48 \mathrm{~mm}$ SL, Trincomalee, Sri Lanka.

Paratype. P.26504-003, 38 mm SL, Phuket, Thailand.

REMARKS. The type description includes an error: holotype P.16511-001 should read P.26511-001.

Pomacentrus spilotoceps Randall, 2002: 174, figures $4,5$.

Paratype. P.31906-001, 59 mm SL, Tongatapu, Tonga.

Pomacentrus xanthosternus Allen, 1991a: 233, figure (p.163).

PARATYPe. P.25248-002, 2 specimens, $64-71 \mathrm{~mm}$ SL, Pulau Putri, Indonesia.

Pomacentrus yoshii Allen and Randall, 2004: 83, figures 7,8 .

Paratypes. P.32463-001, 2 specimens, 50-69 mm SL, Majuro Atoll, Marshall Islands.

Stegastes baldwini Allen and Woods, 1980: 183, figure 7 . 
Paratypes. P.25547-001, 10 specimens, 48-85 mm SL, Clipperton Island, Pacific Ocean.

Stegastes insularis Allen and Emery, 1985: 19, Plate 2 (figures $\mathrm{f}, \mathrm{g}$ )

Holotype. P.26083-044, $83 \mathrm{~mm}$ SL, Christmas Island, Indian Ocean.

PARATYPES. P.10868-001, 65 mm SL; P.10873-001, $55 \mathrm{~mm}$ SL; P.26083-002, 6 specimens, 31-75 mm SL; P.26088-005, 4 specimens, 32-44 mm SL; P.26093-006, $71 \mathrm{~mm}$ SL; P.26110-010, 4 specimens, 22-39 mm SL; P.26120-006, $59 \mathrm{~mm}$ SL, all from Christmas Island, Indian Ocean.

Stegastes lubbocki Allen and Smith, 1992: 114, figure 1.

Holotype. P.30283-001, 49 mm SL, Ascension Island, Atlantic Ocean.

ParatyPes. P.30279-001, 7 specimens, 15-25 mm SL; P.30280-001, 27 mm SL; P.30281-001, 19 mm SL; P.30282-001, 2 specimens, 15-16 $\mathrm{mm} \mathrm{SL;}$ P.30284-001, 16 specimens, 22-51 mm SL, all from Ascension Island, Atlantic Ocean.

Stegastes pelicieri Allen and Emery, 1985: 27, Plate 3 (figures $g, h$ )

Holotype. P.26522-002, $88 \mathrm{~mm}$ SL, Flic en Flac, Mauritius.

PARATYPE. P.26520-002, 3 specimens, 44-65 mm SL, Flic en Flac, Mauritius; P.26522-001, 2 specimens, 45-81 mm SL, Flic en Flac, Mauritius.

\section{Aplodactylidae}

Aplodactylus westralis Russell, 1987: 85, figure 1. Paratypes. P.4728-001, 235 mm SL, Wilson's Inlet, WA, Australia; P.5877-001, 530 mm SL, Busselton, WA, Australia; P.21799-001, $300 \mathrm{~mm}$ SL, Cheynes Beach, WA, Australia; P.25930-001, 335 mm SL, Fremantle, WA, Australia; P.25931001, 347 mm SL, Geographe Bay, WA, Australia.

\section{Cheilodactylidae}

Cheilodactylus pixi Smith, 1980: 2, Plate $1(\mathrm{a}, \mathrm{b})$. Paratype. P.26839-001, 125 mm SL, Algoa Bay, South Africa.

Cheilodactylus rubrolabiatus Allen and Heemstra, 1976: 313, figures 1, 2.

Holotype. P.25225-001, 182 mm SL, Woodman's Point, WA, Australia.

Paratypes. P.4879-001, 233 mm SL, Rottnest Island, WA, Australia; P.5562-001, $201 \mathrm{~mm}$ SL, Rottnest Island, WA, Australia; P.5925-001, 127 mm SL, Trigg Island, WA, Australia; P.10529001, $41 \mathrm{~mm}$ SL, Maud Landing, WA, Australia; P.22244-001, $64 \mathrm{~mm}$ SL, Rottnest Island, WA, Australia; P.22580-001, 112 mm SL, Rockingham, WA, Australia; P.25255-003, 2 specimens, 93-95 mm SL, Cape Naturaliste, WA, Australia; P.25473-001, 35 mm SL, Albany, WA, Australia.

\section{Mugilidae}

Aldrichetta forsteri nonpilcharda Whitley, 1951: 66. HolotyPe. P.2690-001, 45 mm SL, Bunbury, WA, Australia.

Remarks. Whitley's brief description of this subspecies includes reference to a more detailed description and figure (Whitley, 1945). The latter was based on a single specimen, which was designated by Whitley (1951), as the holotype. SYNONYMY. This name is a junior synonym of Aldrichetta forsteri (Valenciennes, 1836).

Moolgarda (Planiliza) ordensis Whitley, 1945: 17, figure 9.

Holotype. P.2758-001, 390 mm SL, Ord River, WA, Australia.

SYNONYMY. This name is a junior synonym of Liza alata (Steindachner, 1892).

\section{Polynemidae}

Polydactylus auratus McKay, 1970: 9.

Holotype. P.16792-001, 115 mm SL, Napier Broome Bay, WA, Australia.

Paratypes. P.16793-001, 109 mm SL, Napier Broome Bay, WA, Australia; P.16794-001, 131 mm SL, Admiralty Gulf, WA, Australia; P.16795001, 100 mm SL, Admiralty Gulf, WA, Australia. SYNONYMY. This name is a junior synonym of Polydactylus multiradiatus (Günther, 1860).

\section{Labridae}

Choerodon cauteroma Gomon and Allen, 1987: 25. Holotype. P.25508-042, 203 mm SL, Exmouth Gulf, WA, Australia.

Paratypes. P.24259-001, 195 mm SL, Kendrew Island, WA, Australia; P.25095-038, 6 specimens, 98-144 mm SL, Exmouth Gulf, WA, Australia; P.25372-001, 3 specimens, 90-104 mm SL, Exmouth Gulf, WA, Australia.

Choerodon gomoni Allen and Randall, 2002c: 110, figures $1,2$.

Paratype. P.31498-003, 88 mm SL, Banggai Islands, Indonesia.

Choerodon sugillatum Gomon, 1987: 19.

PARATYPes. P.25354-023, 6 specimens, 133-149 mm SL, Monte Bello Islands, WA, Australia.

Choerojulis brownfieldi Whitley, 1945: 28, figure 13. Holotype. P.110-001, 125 mm SL, Garden Island, WA, Australia.

SYNONYMY. This species is now known as Halichoeres brownfieldi. 
Cirrhilabrus cenderawasih Allen and Erdmann, 2006a: 90, figures 1, 2, 3 .

Paratype. P.32772-001, 2 specimens, 47-62 mm SL, Cenderawasih Bay, West Papua, Indonesia.

Cirrhilabrus condei Allen and Randall, 1996: 102, figures 1, 2.

PARATYPE. P.28179-002, 52 mm SL, Rabaul, PNG.

Cirrhilabrus joanallenae Allen, 2000: 47, figures 1, 2. Paratypes. P.31523-001, 2 specimens, $41-44 \mathrm{~mm}$ SL, PülaurWeh,-Indonesia.

Cirrhilabrus laboutei Randall and Lubbock, 1982: 2, Plate 1 (figures $a, b$ ).

PARATYPe. P.24952-001, 65 mm SL, Loyalty Islands, New Caledonia.

Cirrhilabrus lineatus Randall and Lubbock, 1982: 6, Plate 1 (figure c).

Paratype. P.27047-001, 22 mm SL, Grand Récif Ouest, New Caledonia.

Cirrhilabrus lubbocki Randall and Carpenter, 1980: 18, figures $2,3,4,5,6$.

Paratypes. P.25517-001, $55 \mathrm{~mm}$ SL, Mactan Island, Philippines; P.25517-002, 45 mm SL, Mactan Island, Philippines.

Cirrhilabrus marjorie Allen, Randall and Carlson, 2003: 114, figures $1,2,3$.

HoLOTYPE. P.32330-003, $58 \mathrm{~mm}$ SL, Wakaya Island, Fiji.

Cirrhilabrus morrisoni Allen, 1999c: 120, figures 1, $2,3,4$.

HoLotyPe. P.31471-001, 47 mm SL, Hibernia Reef, WA, Australia.

PARATYPes. P.31471-002, 3 specimens, $42-43 \mathrm{~mm}$ SL, Hibernia Reef, WA, Australia.

Cirrhilabrus punctatus Randall and Kuiter, 1989: 44, figures $1,2,3,4,5,6,7$.

PARATYPe. P.27073-024, $73 \mathrm{~mm}$ SL, Byron Bay, NSW, Australia.

Cirrhilabrus pylei Allen and Randall, 1996: 105, figures 3,4 .

Paratype. P.31165-001, $47 \mathrm{~mm}$ SL, Normanby Island, PNG.

Cirrhilabrus randalli Allen, 1995d: 14, figures 1, 2. HoLotype. P.27666-047, 85 mm SL, Rowley Shoals, WA, Australia.

Paratypes. P.27666-051, $72 \mathrm{~mm}$ SL, Rowley Shoals, WA, Australia; P.28023-005, 2 specimens, 63-74 mm SL, Rowley Shoals, WA, Australia; P.28027-015, 2 specimens, 52-58 mm SL, Rowley Shoals, WA, Australia; P.29044-021, 6 specimens, 36-68 mm SL, Ashmore Reef, WA, Australia.

Cirrhilabrus rubrimarginatus Randall, 1992: 114, Plate 2a, b, c.
Paratypes. P.24800-001, 2 specimens, $81-84 \mathrm{~mm}$ SL, Espiritu Santo Island, New Hebrides.

Cirrhilabrus tonozukai Allen and Kuiter, 1999: 137, figures $6,7,8$.

Paratype. P.31499-001, 58 mm SL, Kenau Island, Indonesia.

Cirrhilabrus walindi Allen and Randall, 1996: 108, figures $5,6,7$.

Holotype. P.30890-001, 54 mm SL, Kimbe Bay, PNG.

Paratype. P.30890-002, 46 mm SL, Kimbe Bay, PNG.

Conniella apterygia Allen, 1983c: 44, figures 1, 2. HolotyPE. P.27659-006, $55 \mathrm{~mm}$ SL, Rowley Shoals, WA, Australia.

Paratypes. P.27668-003, $59 \mathrm{~mm}$ SL, Rowley Shoals, WA, Australia; P.27668-015, $31 \mathrm{~mm} \mathrm{SL}$ (c/s), Rowley Shoals, WA, Australia.

Coris pictoides Randall and Kuiter, 1982: 160, Plate 1 a; figure 1 .

Paratypes. P.25109-003, $64 \mathrm{~mm}$ SL, Dampier Archipelago, WA, Australia; P.25110-003, 65 mm SL, Dampier Archipelago, WA, Australia.

Dotalabrus alleni Russell, 1988: 10, figure 2; Plate 1 $(\mathrm{c}-\mathrm{d})$.

HoLotype. P.25252-006, $74 \mathrm{~mm}$ SL, Carnac Island, WA, Australia.

PARATYPe. P.25195-004, 2 specimens, 64-72 mm SL, Cape Naturaliste, WA, Australia; P.25251018, 6 specimens, 38-80 mm SL, Rottnest Island, WA, Australia.

Halichoeres chrysus Randall, 1981a: 416, figures 1, 2.

Paratypes. P.26082-004, 3 specimens, 29-53 $\mathrm{mm}$ SL, Christmas Island, Indian Ocean; P.26087-009, 4 specimens, $28-60 \mathrm{~mm}$ SL, Christmas Island, Indian Ocean; P.26099-019, $50 \mathrm{~mm}$ SL, Christmas Island, Indian Ocean; P.26125-007, $42 \mathrm{~mm} \mathrm{SL}$, Christmas Island, Indian Ocean; P.26851-001, 47 mm SL, Cebu, Philippines.

REMARKS. The type description includes an error: P.26850-001 should read P.26851-001.

Halichoeres insularis Allen and Robertson, 1992b: 51, figures 5, 6 .

PARATYPES. P.30390-002, 5 specimens, $46-60 \mathrm{~mm}$ SL, Socorro Island, Mexico.

REMARKS. The type description includes an error: P.30389-002 should read P.30390-002.

Halichoeres malpelo Allen and Robertson, 1992b: 47, figures $1,2$.

Paratypes. P.30389-002, 2 specimens, 107-113 $\mathrm{mm}$ SL, Malpelo Island, Colombia. 
Halichoeres melasmapomus Randall, 1981a: 421, figures 3,4 .

Paratype. P.25233-006, 80 mm SL, Ambon Island, Indonesia.

Labropsis alleni Randall, 1981b: 131, Plate 1 (figure a).

Paratype. P.26906-001， 49 mm SL, Kranket Island, PNG.

Labropsis xanthonota Randall, 1981b: 138, Plate 2 (figures $a, b, c)$.

Paratype. P.26085-003, $100 \mathrm{~mm}$ SL, Christmas Island, Indian Ocean.

Macropharyngodon ornatus Randall, 1978: 753, figures $3 \mathrm{~g}, \mathrm{~h}$.

PARATYPeS. P.24362-001, $108 \mathrm{~mm}$ SL, Kendrew Island, WA, Australia; P.25371-011, $71 \mathrm{~mm} \mathrm{SL}$, North West Cape, WA, Australia.

Paracheilinus cyaneus Kuiter and Allen, 1999: 121, figures 1, 2, 3 .

Paratype. P.31498-001, $49 \mathrm{~mm}$ SL, Banggai Island, Indonesia.

Paracheilinus flavianalis Kuiter and Allen, 1999: 123, figures 4, 5, 6, 7, 18 .

HOLOTYPE. P.30836-007, 43 mm SL, Scott Reef, WA, Australia.

Paratrpes. P.31194-001, 14 specimens, 20-35 mm SL, Seringapatam Reef, WA, Australia.

Paracheilinus waltoni Allen and Erdmann, 2006b: 14, figures $1,2,3$.

Paratype. P.32771-001, 2 specimens, 41-28 mm SL, West Yapen Island, West Papua, Indonesia.

Pictilabrus brauni Hutchins and Morrison, 1996: 39, figures $1,4,7$.

Holotype. P.30941-001, 74 mm SL, Cheynes Beach, WA, Australia.

Paratypes. P.30940-001, 58 mm SL; P.30941-002, $70 \mathrm{~mm}$ SL; P.30942-001 (c/s), $61 \mathrm{~mm}$ SL, all from Cheynes Beach, WA, Australia.

Pictilabrus viridis Russell, 1988: 38, figure 8; Plate 4 $(\mathrm{e}, \mathrm{f})$.

Paratypes. P.24859-001, 2 specimens, 96-121 mm SL, Albany, WA, Australia; P.25178-004, 2 specimens, 75-80 mm SL, Rottnest Island, WA, Australia.

Pseudocheilinus ocellatus Randall, 1999: 21, figure 3; Plates 1 (h), 2 (a).

Paratype. P.29638-021, $47 \mathrm{~mm}$ SL, Lihou Reef, Qld., Australia

Pseudojuloides elongatus Ayling and Russell, 1977: 174, figures 8, 9, 10.

Paratypes. P.25110-001, 3 specimens, $88-97 \mathrm{~mm}$ SL, Dampier Archipelago, WA, Australia;
P.25318-007, $107 \mathrm{~mm}$ SL, Houtman Abrolhos, WA, Australia; P.25318-008, 112' mm SL, Houtman Abrolhos, WA, Australia.

Suezichthys cyanolaemus Russell, 1985: 13, Plate 2 (figures c, d); figure 7a.

Paratypes. P.25311-002, 5 specimens, 39-67 mm SL, Houtman Abrolhos, WA, Australia; P.25316003, $43 \mathrm{~mm}$ SL, Houtman Abrolhos, WA, Australia.

Thalassoma robertsoni Allen, 1995e: 75, figures 1, 2. HoLotyPe. P.30993-001, 72 mm SL, Clipperton Island, Pacific Ocean.

Paratypes. P.30993-002, 15 specimens, 12-74 mm SL, Clipperton Island, Pacific Ocean.

Xyrichtys perlas Wellington, Allen and Roberston, 1994: 49, figures 1, 2, 3 .

Paratype. P.30178-001, 2 specimens, 36-42 mm SL, Perlas Islands, Panama.

Xyrichtys victori Wellington, 1992: 1054, figures 1, 2.

Paratypes. P.30177-001, 3 specimens, $31-139 \mathrm{~mm}$ SL, Marchena Island, Galapagos Islands.

Xyrichtys wellingtoni Allen and Robertson, 1995b: 80 , figure 1.

HoLOTYPE. P.30994-001, 72 mm SL, Clipperton Island, Pacific Ocean.

\section{Odacidae}

Odax cyanoallix Ayling and Paxton, 1983: 96, figures 1,3 .

Paratypes. P.26648-001, 3 specimens, 91-259 mm SL, Three Kings Island, New Zealand.

Parodax caninus Scott, 1976: 367, figure 6.

HoLOTYPe. P.25346-002, 74 mm SL, Carnac Island, WA, Australia.

PARATYPes. P.25251-019, $72 \mathrm{~mm}$ SL, Rottnest Island, WA, Australia; P.25252-009, $59 \mathrm{~mm} \mathrm{SL}$, Carnac Island, WA, Australia; P.25343-010, 48 mm SL, Fremantle, WA, Australia; P.25498-001, $80 \mathrm{~mm}$ SL, Rottnest Island, WA, Australia.

SYNONYMY. This species is now known as Siphonognathus caninus.

Siphonognathus tanyourus Gomon and Paxton, 1986: 51, Plate $6 \mathrm{~d}$, e; figure 21.

PaRATYPes. P.28296-028, 7 specimens, $45-72 \mathrm{~mm}$ SL, Recherche Archipelago, WA, Australia; P.28300-018, 83 mm SL, Lucky Bay, WA, Australia.

\section{Scaridae}

Scarus chameleon Choat and Randall, 1986: 199, Plates 3 (figures c, d), 9 (figures $d, e, f, g, h$ ) 
Paratype. P.28275-001, 168 mm SL, Lizard Island, Qld., Australia

\section{Opistognathidae}

Opistognathus alleni Smith-Vaniz, 2004: 210, figure 1.

HolotyPe. P.27590-030, $74 \mathrm{~mm}$ SL, Houtman Abrolhos, WA, Australia.

PARATYPES. P.27590-002, 11 specimens, $47-72 \mathrm{~mm}$ SL;-P.27595=003, 9 specimens, $45-51 \mathrm{~mm} \mathrm{SL}$; P.27587-003, $54 \mathrm{~mm}$ SL, all from Houtman Abrolhos, WA, Australia.

Opistognathus galapagensis Allen and Robertson, 1991a: 47, figures 1, 2.

ParatyPe. P.30227-001, $132 \mathrm{~mm}$ SL, Isla Isabella, Galapagos Islands.

Opistognathus reticeps Smith-Vaniz, 2004: 220, figure 11.

Paratypes. P.24227-001, $113 \mathrm{~mm}$ SL, Napier Broome Bay, WA, Australia.

Opistognathus rosenblatti Allen and Robertson, 1991a: 50, figures 7, 8, 9, 10.

ParatyPes. P.30228-001, 3 specimens, 41-72 mm SL, Isla San Pedro Martir, Mexico.

Opistognathus stigmosus Smith-Vaniz, 2004: 215, figure 7.

Paratypes. P.27467-004, $58 \mathrm{~mm}$ SL, Escape Reef, Qld., Australia; P.29641-001, 63 mm SL, Lihou Reef, Qld., Australia

Opistognathus verecundus Smith-Vaniz, 2004: 218, figure 9.

Paratypes. P.30593-001, 4 specimens, 34-37 mm SL, Coburg Peninsula, NT, Australia.

Tandya reticulata McKay, 1969: 1, figure 1.

HoLOTYPE. P.15758-001, $385 \mathrm{~mm}$ SL, Broome, WA, Australia.

SynONYMY. This species is now known as Opistognathus reticulatus.

\section{Pinguipedidae}

Parapercis biordinis Allen, 1976a: 28, figure 3. Holotrpe. P.25206-001, $70 \mathrm{~mm}$ SL, Point Cloates, WA, Australia.

Parapercis multiplicata Randall, 1984: 48, figures 4,5 .

Paratype. P.25375-001, $55 \mathrm{~mm} \mathrm{SL}$, North West Cape, WA, Australia.

Parapercis (Neopercis) naevosa Serventy, 1937: 72, Plate 2.

Holotype. P.1158-001, $116 \mathrm{~mm} \mathrm{SL,} \mathrm{Great}$ Australian Bight, WA, Australia.
SYNONYMY. This name is a junior synonym of Parapercis allporti (Günther, 1876).

\section{Percophidae}

Bembrops aethalea McKay, 1971: 42, figure 2.

HoLOTYPE. P.19144-001, $177 \mathrm{~mm}$ SL, northwestern Australia, Indian Ocean.

Paratypes. P.19142-001, 179 mm SL; P.19143-001, $180 \mathrm{~mm}$ SL; P.19145-001, $183 \mathrm{~mm}$ SL; P.19146001, $138 \mathrm{~mm} \mathrm{SL;} \mathrm{P.19147-001,} 144 \mathrm{~mm} \mathrm{SL;}$ P.19148=001,-179-mm-SL;-P.19149-001, 175-mm SL; P.19150-001, $170 \mathrm{~mm}$ SL; P.19151-001, 121 $\mathrm{mm} \mathrm{SL}$, all from north-western Australia, Indian Ocean.

Bembrops indica McKay, 1971: 43, figure 3. HoLOTYPE. P.19138-001, $161 \mathrm{~mm}$ SL, northwestern Australia, Indian Ocean.

PARATYPES. P.19137-001, $158 \mathrm{~mm}$ SL; P.19139-001, 80 mm SL; P.19140-001, 192 mm SL; P.19141-001, 119 mm SL; P.19162-001, 99 mm SL; P.19163-001, $99 \mathrm{~mm}$ SL, all from north-western Australia, Indian Ocean..

SYNONYMY. This name is a junior synonym of Bembrops filiferus Gilbert, 1905.

Branchiopsaron ozawai McKay, 1971: 41, figure 1. HOLOTYPE. P.19153-001, 106 mm SL, northwestern Australia, Indian Ocean.

ParatyPes. P.19152-001, $108 \mathrm{~mm}$ SL; P.19154-001, 86 mm SL; P.19155-001, 93 mm SL; P.19156-001, $70 \mathrm{~mm}$ SL, P.19157-001, 65 mm SL; P.19158-001, $57 \mathrm{~mm} \mathrm{SL}$, all from north-western Australia, Indian Ocean.

SYNONYMY. This species is now known as Acanthaphritis ozawai.

Chrionema chlorotaenia McKay, 1971: 44, figure 4. Holotype. P.19136-001, $194 \mathrm{~mm} \mathrm{SL}$, northwestern Australia, Indian Ocean.

ParatyPes. P.19129-001, 187 mm SL; P.19130-001, $187 \mathrm{~mm} \mathrm{SL} ;$ P.19131-001, $175 \mathrm{~mm}$ SL; P.19132001, $193 \mathrm{~mm}$ SL; P.19133-001, $169 \mathrm{~mm} \mathrm{SL;}$ P.19134-001, $156 \mathrm{~mm}$ SL; P.19135-001, $131 \mathrm{~mm}$ SL, all from north-western Australia, Indian Ocean.

\section{Creediidae}

Creedia alleni Nelson, 1983: 29, figure 1.

Holotype. P.25808-005, $40 \mathrm{~mm}$ SL, Fremantle, WA, Australia.

PARATYPe. P.25346-028, $36 \mathrm{~mm}$ SL, Carnac Island, WA, Australia; P.25347-001, 36 mm SL, Carnac Island, WA, Australia.

Limnichthys fasciatus major Whitley, 1945: 31. HoLOTYPE. P.121-001, $50 \mathrm{~mm}$ SL, Garden Island, WA, Australia. 
SYNONYMY. This name is a junior synonym of Limnichthys fasciatus Waite, 1904.

\section{Uranoscopidae}

Gnathagnus cribratus Kishimoto, 1989: 305, figure 1.

HoLotype. P.28071-009, 197 mm SL, Rowley Shoals, WA, Australia.

PARATYPES. P.28071-026, 2 specimens, 124-131 mm SL, Rowley Shoals, WA, Australia; P.28086007, $120 \mathrm{~mm}$ SL, Rowley Shoals, WA, Australia. SynONYMY. This species is now known as Xenocephalus cribratus.

Gnathagnus elongatus australiensis Kishimoto, 1989: 310, figure 2.

PARATYPES. P.19119-001, 213 mm SL; P.28072-006, 2 specimens, 153-156 mm SL; P.28086-001, 2 specimens, $140-195 \mathrm{~mm} \mathrm{SL}$, all from vicinity of Rowley Shoals, WA, Australia

SYNONYMY. This name is a junior synonym of Xenocephalus australiensis (Kishimoto, 1989).

Ichthyscopus barbatus Mees, 1960: 49, figures 3, 4. HOLOTYPE. P.4338-001, 34 mm SL, Rottnest Island, WA, Australia.

PARATYPES. P.672-001, $200 \mathrm{~mm}$ SL, Woodman's Point, WA, Australia; P.1296-001, 147 mm SL, Busselton, WA, Australia; P.3488-001, 32 mm SL, Dunsborough, WA, Australia; P.4424-001, 300 mm SL, Eagle Bay, WA, Australia; P.4431-001, 2 specimens, 105-124 mm SL, Rottnest Island, WA, Australia.

Ichthyscopus insperatus Mees, 1960: 54, figures 8, 9. HOLOTYPE. P.3638-001, 170 mm SL, Roebuck Bay, WA, Australia.

Ichthyscopus spinosus Mees, 1960: 48, figures 1, 2. HoLOTYPE. P.3639-001, 258 mm SL, near Broome, WA, Australia.

\section{Champsodontidae}

Champsodon pantolepis Nemeth, 1994: 361, figure 11.

HoLotyPe. P.28098-003, $128 \mathrm{~mm} \mathrm{SL}$, north of Dampier, WA, Australia.

REMARKS. The type description includes an error: P.28098003 should read P.28098-003.

Champsodon sagittus Nemeth, 1994: 363, figure 12. Holotype. P.25400-019, 65 mm SL, Browse Island, WA, Australia.

PARATYPES. P.25395-017, 52 mm SL, Rowley Shoals, WA, Australia; P.25400-020, 5 specimens (1 c/s), 52-65 mm SL, Browse Island, WA, Australia; P.28057-012, 54 mm SL, Rowley Shoals, WA, Australia; P.28075-003, 54 mm SL, Rowley Shoals, WA, Australia; P.28076-007, 60 mm SL, Rowley Shoals, WA, Australia; P.29304005, 11 specimens, 55-77 mm SL, Joseph Boneparte Gulf, WA, Australia.

REMARKS. The type description includes an error: paratypes P.25395-0017 should read P.25395-017.

\section{Bovichthyidae}

Halaphritis platycephala Last, Balushkin and Hutchins, 2002: 435, figure 1.

PaRATYPes. P.27545-010, 2 specimens, 74-99 mm SL, Port Esperance, Tas., Australia; P.27549-017, 162 mm SL, Port Arthur, Tas., Australia

\section{Blenniidae}

Cirripectes alleni Williams, 1993: 61, figures 1, 2, 3. Holotype. P.30309-018, 51 mm SL, Cassini Island, WA, Australia.

ParatYPes. P.29081-014, 32 specimens, 22-54 mm SL, Adele Island, WA, Australia; P.30307-009, 10 specimens, 30-64 mm SL, Long Reef, WA, Australia; P.30309-014, 6 specimens, 24-55 mm SL, Cassini Island, WA, Australia; P.30311-032, 5 specimens, 39-49 mm SL, Monge Island, WA, Australia.

Cirripectes chelomatus Williams and Maugé, 1984: 1140, figure 2.

Paratypes. P.27754-001, 2 specimens, $58-70 \mathrm{~mm}$ SL, One Tree Island, Qld., Australia

Cirripectes gilberti Williams, 1988: 41, Plate 3 (figures d, e); figures 14, 15.

PARATYPeS. P.29331-012, 2 specimens, 32-62 $\mathrm{mm}$ SL, Christmas Island, Indian Ocean.

Cirripectes hutchinsi Williams, 1988: 44, Plate 3 (figures $\mathrm{f}, \mathrm{g}, \mathrm{h}$ ).

HolotyPe. P.25744-003, $89 \mathrm{~mm} \mathrm{SL}$, Rottnest Island, WA, Australia.

Paratypes. P.4647-001, 73 mm SL, Point Quobba, WA, Australia; P.4652-001, 98 mm SL, Houtman Abrolhos, WA, Australia; P.25317-002, 2 specimens, 90-106 mm SL, Houtman Abrolhos, WA, Australia; P.25374-042, $69 \mathrm{~mm}$ SL, North West Cape, WA, Australia; P.25744-005, $53 \mathrm{~mm}$ SL, Rottnest Island, WA, Australia; P.25758-010, $73 \mathrm{~mm}$ SL, Rottnest Island, WA, Australia; P.26072-003, 2 specimens, 32-52 mm SL, Houtman Abrolhos, WA, Australia; P.26626-001, $76 \mathrm{~mm} \mathrm{SL}$, North West Cape, WA, Australia; P.26657-009, 3 specimens, 34-70 mm SL, Shark Bay, WA, Australia.

Dasson duperreyi Whitley, 1945: 31.

HoLoryPE. P.2152-001, 89 mm SL, Shark Bay, WA, Australia.

Paratype. P.2153-001, 50 mm SL, Shark Bay, WA, Australia. 
REMARKS. Although the type description refers to 'several specimens in the W.A. Museum', under the heading of paratypes, Whitley only provided a registration number and counts for the single specimen cited above. The location of any other specimens examined is unknown.

SYNONYMY. This name is a junior synonym of Petroscirtes breviceps (Valenciennes, 1836).

Ecsenius alleni Springer, 1988: 81, Plate 12 (figure 2); figure 42 .

Holotype. P.27666-017, 28 mm SL, Rowley Shoals, WA, Australia.

PARATYPES. P.27656-021, 32 mm SL; P.27658-013, 27 mm SL; P.27659-009, 4 specimens, 24-32 mm SL; P.27662-026, 2 specimens, $30-33 \mathrm{~mm} \mathrm{SL;}$ P.27663-010, $26 \mathrm{~mm}$ SL; P.27665-001, 4 specimens, 28-32 mm SL; P.27668-014, $26 \mathrm{~mm}$ SL; P.28022-048, 19 specimens, 22-34 mm SL, all from Rowley Shoals, WA, Australia.

REMARKS. The type description includes an error: paratypes P.27660-009 should read P.27659-009.

Ecsenius axelrodi Springer, 1988: 84, Plate 10 (figures 1, 2, 3); figure 44 .

Paratypes. P.28182-004, 2 specimens, $20-34 \mathrm{~mm}$ SL, Rabaul, PNG.

Ecsenius caeruliventris Springer and Allen, 2004: 3, figures 1a, b, c, 2a.

PARATYPES. P.31492-001, 4 specimens, $16-23 \mathrm{~mm}$ SL, Batudaka Island, Indonesia.

Ecsenius oculatus Springer, 1988: 60, Plate 7 (figure 1); figure 32c.

HolotyPE. P.25368-023, $44 \mathrm{~mm}$ SL, North West Cape, WA, Australia.

PARATYPES. P.25368-037, 3 specimens, 32-37 mm SL, North West Cape, WA, Australia; P.25374-018, 9 specimens, 26-33 mm SL, North West Cape, WA, Australia; P.25813-050, 26 mm SL, South Muiron Island, WA, Australia; P.26102-005, $26 \mathrm{~mm} \mathrm{SL,} \mathrm{Christmas} \mathrm{Island,}$ Indian Ocean; P.26110-004, 24 specimens, 18$36 \mathrm{~mm}$ SL, Christmas Island, Indian Ocean; P.26670-006, 3 specimens, 39-47 mm SL, Dirk Hartog Island, WA, Australia; P.27961-014, 53 mm SL, Point Quobba, WA, Australia; P.27970-041, $39 \mathrm{~mm}$ SL, Point Quobba, WA, Australia.

Remarks. The type description lists the same registration number for both the holotype and one lot of paratypes. We have amended the registration number of the paratypes to P.25368037.

Ecsenius shirleyae Springer and Allen, 2004: 8, figures 1d, 2b.

Paratypes. P.32602-001, 2 specimens, 22-29 mm SL, Pulau Pari, Indonesia.
Ecsenius taeniatus Springer, 1988: 112, figure 60. Paratype. P.24913-001, 32 mm SL, Goodenough Island, PNG.

Graviceps alexanderi Whitley, 1945: 33.

HoLOTYPE. P.671-001, 32 mm SL, Fremantle, WA, Australia.

Paratypes. P.398-001, $47 \mathrm{~mm}$ SL, Houtman Abrolhos, WA, Australia; P.671-002, 34 mm SL, Fremantle, WA, Australia.

REMARKS. The type description provides the details-of-three-specimens from the Houtman Abrolhos, P.395-001, which are referred to as 'perhaps the male of the species'. These specimens, along with five others (P.396-001), were treated by Whitley as comparative material only and are therefore not part of the type series (e.g. he compares the fin colours of P.395-001 with those of 'the paratypes').

SYNONYMY. This name is a junior synonym of Omobranchus germaini (Sauvage, 1883).

Meiacanthus (Meiacanthus) limbatus Smith-Vaniz, 1987: 19, figure 7c.

HoLoTYPE. P.27826-018, 37 mm SL, Manus, PNG.

Meiacanthus (Meiacanthus) naevius Smith-Vaniz, 1987: 20, figure 7d.

HoLOTYPE. P.28030-017, $36 \mathrm{~mm}$ SL, Rowley Shoals, WA, Australia.

Meiacanthus (Allomeiacanthus) urostigma SmithVaniz, Satapoomin and Allen, 2001: 29, figures 6, $7,8$.

Paratypes. P.31527-001, 2 specimens, $31-37 \mathrm{~mm}$ SL, Pulau Weh, Indonesia.

Parablennius tasmaniensis postoculomaculatus Bath and Hutchins, 1986: 187, figures 24-29.

Paratypes. P.25850-008, 10 specimens, $16-45 \mathrm{~mm}$ SL, Houtman Abrolhos, WA, Australia.

SYNONYMY. This species is now known as Parablennius postoculomaculatus.

Salarias ramosus Bath, 1992: 230, figures 7, 8, 9, 10. Holotype. P.27599-009, 53 mm SL, North West Cape, WA, Australia.

PARATYPes. P.30057-002, 3 specimens, 56-61 mm SL, Barrow Island, WA, Australia.

\section{Tripterygiidae}

Axoclinus nigricaudus Allen and Robertson, 1991b: 79 , figure 1 .

PARATYPe. P.30233-001， $31 \mathrm{~mm}$ SL, Baja California, Mexico.

Axoclinus rubinoffi Allen and Robertson, 1992b: 53, figure 1.

Paratype. P.30389-001, 22 mm SL, Malpelo Island, Colombia. 
[Brachynectes fasciatus Scott, 1957: 181, figure 1.

ReMARKs. Two specimens, P.4846 were recorded as paratypes in the WAM Fish Catalogue, however Scott (1957) clearly states that only one specimen, the holotype, was used for the type description. These other specimens were part of the additional material examined and therefore have no type status. They are mentioned here to avoid potential confusion caused by the original entries in the WAM Fish Catalogue.

Ceratobregma helenae Holleman, 1987: 175, figure 2.

HolotyPe. P.26098-012, 29 mm SL, Christmas Island, Indian Ocean.

Paratypes. P.26080-017, 2 specimens, $26-28 \mathrm{~mm}$ SL; P.26090-011, $29 \mathrm{~mm}$ SL; P.26092-021, 5 specimens, 20-28 $\mathrm{mm}$ SL; P.26098-007, 3 specimens, 27-29 mm SL; P.26110-015, 2 specimens, 24-29 mm SL; P.26113-018, $26 \mathrm{~mm}$ SL; P.26122-011, $27 \mathrm{~mm} \mathrm{SL}$, all from Christmas Island, Indian Ocean.

Crocodilichthys gracilis Allen and Robertson, 1991b: 79, figure 2.

Paratype. P.30231-001， 46 mm SL, Baja California, Mexico.

Enneanectes reticulatus Allen and Robertson, 1991b: 80, figure 3.

Paratype. P.30232-001, $35 \mathrm{~mm}$ SL, Baja California, Mexico.

Enneapterygius bichrous Fricke, 1994: 195, figure 30.

PARATYPES. P.27663-025, $20 \mathrm{~mm}$ SL, Rowley Shoals, WA, Australia; P.28025-039, 13 specimens, 19-23 mm SL, Rowley Shoals, WA, Australia; P.28031-029, 22 mm SL, Rowley Shoals, WA, Australia; P.29048-008, 3 specimens, 14-18 mm SL, Ashmore Reef, WA, Australia; P.29054-012, 19 mm SL, Ashmore Reef, WA, Australia; P.29624-077, 4 specimens, $15-18 \mathrm{~mm}$ SL, Madang, PNG.

REMARKs. The type description includes three errors: paratype P.27663-025 is listed twice, but with different measurements ( 15 and $20 \mathrm{~mm} \mathrm{SL}$ ). The WAM specimen is $20 \mathrm{~mm}$ SL. Paratype P.28031-029 is listed for this species but also appears under 'other material' for Helcogramma striata (Fricke, 1994: 440). Paratypes P.29048-008 are listed first as two paratype specimens and later as three 'other material' specimens. The lot housed at WAM includes three specimens (as listed on the original data sheet).

SYNONYMY. This name is a junior synonym of Enneapterygius flavoccipitis Shen, 1994.

Enneapterygius gracilis Fricke, 1994: 209, figure 34. ParATYPES. P.27274-041, 17 specimens, $15-23 \mathrm{~mm}$
SL; P.28060-016, 5 specimens, $18-22 \mathrm{~mm} \mathrm{SL;}$ P.28417-025, 7 specimens, 12-17 mm SL, all from near Broome, WA, Australia.

Enneapterygitus larsonae Fricke, 1994: 235, figures $44,45$.

Paratypes. P.25308-016, $22 \mathrm{~mm}$ SL, Houtman Abrolhos, WA, Australia; P.25317-011, 2 specimens, 31-32 mm SL, Houtman Abrolhos, WA, Australia; P.26668-011, 5 specimens, 20-26 mm SL, Shark Bay, WA, Australia; P.28416-029, 2 specimens, $17-18 \mathrm{~mm}$ SL, Broome, WA, Australia; P.29042-027, 5 specimens, 13-24 mm SL, Ashmore Reef, WA, Australia; P.29883-004, $26 \mathrm{~mm}$ SL, Houtman Abrolhos, WA, Australia; P.29884-015, $27 \mathrm{~mm}$ SL, Houtman Abrolhos, WA, Australia; P.29887-006, 29 mm SL, Houtman Abrolhos, WA, Australia.

REMARKS. The type description includes an error: P.25308-014 should read P.25308-016.

Enneapterygius mirabilis Fricke, 1994: 251, figure 48.

Paratypes. P.28531-012, $18 \mathrm{~mm}$ SL, Great Barrier Reef, Qld; P.28534-024, 15 mm SL, Great Barrier Reef, Qld., Australia

Enneapterygius similis Fricke, 1997: 326, figure 68. PARATYPE. P.30416-011, $21 \mathrm{~mm}$ SL, Sipadan, Malaysia.

Enneapterygius triserialis Fricke, 1994: 280, figures $56,57$.

Paratypes. P.29627-034, 4 specimens, $19-23 \mathrm{~mm}$ SL, Holmes Reefs, Qld., Australia

Helcogramma lacuna Williams and Howe, 2003: 165, figures 13, 14 .

Paratypes. P.26507-016, 2 specimens, 33-35 mm SL, Similan Islands, Thailand.

Norfolkia cristata Kuiter, 1986b: 92, figures 4, 5 . Paratypes. P.27136-016, 3 specimens, $28-35 \mathrm{~mm}$ SL, Victor Harbour, SA, Australia.

Norfolkia incisa Kuiter, 1986b: 93, figures 6, 7. Paratypes. P.26005-010, 7 specimens, 21-28 mm SL, Recherche Archipelago, WA, Australia; P.27123-012, 4 specimens, 24-28 mm SL, Wilson's Promontory, Vic., Australia SYNONYMY. This species is now known as Trinorfolkia incisa.

Norfolkia leeuwin Fricke, 1994: 471, figure 106. Paratype. P.25308-014, 24 mm SL, Houtman Abrolhos, WA, Australia.

REMARKS. The type description includes an error: P.25308-000 should read P.25308-014.

Taboguilla signata Allen and Robertson, 1991b: 81, figures 4,5 . 
Paratype. P.30229-001, 39 mm SL, Punta Mariato, Panama.

SYNONYMY. This name is a junior synonym of Lepidonectes clarkhubbsi Bussing, 1991.

Vauclusella calva Whitley, 1944a: 272.

Holotype. P.1052-001, 37 mm SL, Cottesloe, WA, Australia.

ParatyPe. P.1052-002, $39 \mathrm{~mm}$ SL, Cottesloe, WA, Australia.

SYNONYMY. This name is a junior synonym of Helcogramma decurrens McCulloch and-Waite, 1918.

\section{Clinidae}

Clinus eckloniae McKay, 1970: 16, figure 1. HoLotyPe. P.16785-001, 73 mm SL, Albany, WA, Australia.

Paratype. P.16786-001, $39 \mathrm{~mm}$ SL, Rottnest Island, WA, Australia.

SYNONYMY. This species is now known as Heteroclinus eckloniae.

Heteroclinus kuiteri Hoese and Rennis, 2006: 21, figures 1, 2, 3 .

Paratypes. P.25767-010, 3 specimens, 38-47 mm SL, Recherche Archipelago, WA, Australia; P26599-004, 4 specimens, 34-48 mm SL, Cosy Corner, WA, Australia.

Petraites equiradiatus Milward, 1960: 134, figure.

HoLOTYPE. P.4472-001, 97 mm SL, Rottnest Island, WA, Australia.

SYNONYMY. This species is now known as Heteroclinus equiradiatus.

\section{Ophiclinidae}

Ophiclinops hutchinsi George and Springer, 1980: 9 , figure 2.

HolotyPe. P.26004-008, 89 mm SL, Lucky Bay, WA, Australia.

PARATYPes. P.26003-016, $31 \mathrm{~mm}$ SL, Lucky Bay, WA, Australia; P.26454-001, 2 specimens, 50-52 mm SL, Lucky Bay, WA, Australia.

Ophiclinus brevipinnis George and Springer, 1980: 17, figure 6.

Paratype. P.26000-022, $59 \mathrm{~mm}$ SL, Lucky Bay, WA, Australia.

Ophiclinus ningulus George and Springer, 1980: 22, figure 10.

Holotype. P.26009-015, 44 mm SL, Lucky Bay, WA, Australia.

Ophiclinus pectoralis George and Springer, 1980:

23, figures 11,12 .

HOLOTYPE. P.25770-006, $52 \mathrm{~mm}$ SL, Recherche Archipelago, WA, Australia.
Paratypes. P.25758-015, $31 \mathrm{~mm}$ SL, Rottnest Island, WA, Australia; P.26008-008, 2 specimens, 31-40 mm SL, Recherche Archipelago, WA, Australia.

\section{Callionymidae}

Callionymus (Spinicapitichthys) csiro Fricke, 1983: 457, figure 135.

HoLOTYPE. P.11212-001, 23 mm SL, Carnarvon, WA, Australia.

SYNONYMY. This name is a junior synonym-of Callionymus draconis Nakabo, 1977.

Callionymus (Calliurichthys) margaretae australis Fricke, 1983: 402, figures 118, 119.

Paratype. P.11841-001, 62 mm SL, Shark Bay, WA, Australia.

SYNONYMY. This species is now known as Callionymus australis.

Calliurichthys goodladi Whitley, 1944a: 270.

HolotyPe. P.2528-001, $131 \mathrm{~mm}$ SL, Cheyne Beach, WA, Australia

Paratypes. P.2541-001 to P.2543-001, 3 specimens, 117-126 mm SL, Shark Bay, WA, Australia.

SYNONYMY. This species is now known as Pseudocalliurichthys goodladi.

Synchiropus grandoculis Fricke, 2000: 69, figure 33. HoLotype. P.30418-001, $128 \mathrm{~mm}$ SL, Rowley Shoals, WA, Australia.

REMARKS. The type description includes an error: P.20418-001 should read P.30418-001.

Synchiropus (Synchiropus) moyeri Zaiser and Fricke, 1985: 389, figures 1, 2.

Paratype. P.28366-001, $33 \mathrm{~mm}$ SL, Izu Islands, Japan.

Synchiropus (Synchiropus) picturatus occidentalis Fricke, 1983: 653, figure 202.

HoLOTYPE. P.22027-001, 39 mm SL, Dampier Archipelago, WA, Australia.

Paratypes. P.22028-001, $38 \mathrm{~mm}$ SL, Dampier Archipelago, WA, Australia; P.22029-001, $40 \mathrm{~mm}$ SL, Dampier Archipelago, WA, Australia.

SYNONYMY. This species is now known as Synchiropus occidentalis.

\section{Draconettidae}

Liopsaron insolitus McKay, 1971: 45, figure 5. HOLOTYPE. P.19164-001, 93 mm SL, north-western Australia, Indian Ocean.

PARATYPES. P.19165-001, 87 mm SL, north-western Australia, Indian Ocean; P.19166-001, 71 mm SL, north-western Australia, Indian Ocean.

SYNONYMY. This species is now known as Centrodraco insolitus. 


\section{Gobiidae}

Amblyeleotris arcupinna Mohlmann and Munday, 1999: 59, figures 1, 2.

Paratype. P.31206-001， $70 \mathrm{~mm}$ SL, Cape Heussner, PNG (missing).

Amblyeleotris randalli Hoese and Steene, 1978: 382, figures $1,2,3$.

PaRATYPe. P.25235-021, 58 mm SL, Ambon Island, Indonesia.

Amblyeleotris rubrimarginata Mohlmann and Randall, 2002: 216, figures 1, 2, 3.

PARATYPES. P.31767-001, 49 mm SL, Lizard Island, Qld; P.31768-001, 34 mm SL, Lizard Island, Qld., Australia

Asterropteryx bipunctatus Allen and Munday, 1995: 99, figures $1,2$.

Holotype. P.30346-007, $31 \mathrm{~mm}$ SL, Madang, PNG.

Paratypes. P.28170-034, 2 specimens, $20-28 \mathrm{~mm}$ SL, Rabaul, PNG; P.28181-016, 24 mm SL, Rabaul, PNG; P.30357-019, 16 mm SL, Madang, PNG; P.30369-010, 25 mm SL, Madang, PNG; P.30406-005, 3 specimens, 17-21 mm SL, Sibuan Island, Indonesia; P.30620-013, $23 \mathrm{~mm} \mathrm{SL}$, Madang, PNG; P.30804-017, 22 mm SL, Kakaban Island, Indonesia.

REMARKS. The type description includes an error: paratype P.30620 should read P.30620-013.

Asterropteryx striatus Allen and Munday, 1995: 101, figures 3,4 .

Holotype. P.30376-023, $23 \mathrm{~mm} \mathrm{SL}$, Madang, PNG.

Paratype. P.30719-041, 23 mm SL, Maumere Bay, Indonesia.

Awaous (Awaous) acritosus Watson, 1994: 372, figure 1.

Paratypes. P.27395-006, 3 specimens, $93-114 \mathrm{~mm}$ SL, Hohora Creek, PNG; P.28545-005, 102 mm SL, Hilda Creek, Qld; P.28546-006, 6 specimens, 40-92 mm SL, Cooper Creek, Qld., Australia

Bryaninops amplus Larson, 1985: 66, figures 5, 6 .

PARATYPES. P.25109-002, 3 specimens, $8-16 \mathrm{~mm}$ SL, Dampier Archipelago, WA, Australia; P.28039-001, 4 specimens, 16-24 mm SL, Lizard Island, Qld., Australia

Bryaninops isis Larson, 1985: 89, figure 17. PARATYPES. P.28441-001, $18 \mathrm{~mm}$ SL, Lizard Island, Qld; P.28442-001, 16 mm SL, Lizard Island, Qld., Australia

Bryaninops loki Larson, 1985: 81, figures 13, 14. Paratypes. P.28042-001, 2 specimens, 22-23 mm SL, Lizard Island, Qld., Australia
Bryaninops natans Larson, 1985: 77, figures 10, 11, 12.

Paratypes. P.27666-012, 12 specimens, 11-17 mm SL, Rowley Shoals, WA, Australia; P.28041-001, 8 specimens, $14-17 \mathrm{~mm} \mathrm{SL}$, Gulf Of Aqaba, Israel.

Bryaninops nexus Larson, 1987: 139, figures 1, 2, 3. PARATYPES. P.28030-030, 5 specimens, $11-19 \mathrm{~mm}$ SL, Rowley Shoals, WA, Australia.

Bryaninops tigris Larson, 1985: 70, figures 7, 8.

PARATYPe. P.28040-001, 17 mm SL, Lizard Island, Qld., Australia

Cristatogobius rubripectoralis Akihito, Meguro and Sakamoto, 2003: 117, figures 1, 2.

Paratypes. P.31776-001, 2 specimens, $21-47 \mathrm{~mm}$ SL, Saunders Beach, Qld (missing).

Drombus halei lepidothorax Whitley, 1945: 37. SYNTYPE. P.2667-001 to P.2673-001, 7 specimens, 29-45 mm SL, Denham, WA, Australia.

SYNONYMY. This species is now known as Drombus lepidothorax.

Echinogobius hayashii Iwata, Hosoya and Niimura, 1998: 115, figures $1,2$.

Paratype. P.30883-002, 86 mm SL, Seringapatam Reef, Indian Ocean.

Eviota bimaculata Lachner and Karnella, 1980: 91, figures $51,52$.

Holotype. P.10110-001, 19 mm SL, Rottnest Island, WA, Australia.

Paratypes. P.10111-001, $20 \mathrm{~mm}$ SL, Rottnest Island, WA, Australia; P.10112-001, 18 mm SL, Rottnest Island, WA, Australia; P.10129-001, 22 mm SL, Rottnest Island, WA, Australia; P.25725019, 4 specimens, 19-22 mm SL, Rottnest Island, WA, Australia; P.10445-001 to P.10448-001, 4 specimens, $19-23 \mathrm{~mm} \mathrm{SL}$, Garden Island, WA, Australia.

Eviota fasciola Karnella and Lachner, 1981: 268, figures 2,3 .

Paratypes. P.26843-001, 5 specimens, $11-17 \mathrm{~mm}$ SL, One Tree Island, Qld., Australia

Eviota mikiae Allen, 2001c: 128, figures 3, 4.

Paratypes. P.31527-003, 8 specimens, $13-18 \mathrm{~mm}$ SL, Pulau Weh, Indonesia.

Eviota raja Allen, 2001c: 126, figure 1.

Paratypes. P.31780-002, 9 specimens, $13-24 \mathrm{~mm}$ SL, Raja Ampat Islands, Indonesia.

REMARKS. The type description includes an error: P.31780-001 should read P.31780-002.

Eviota sigillata Jewett and Lachner, 1983: 799, figure 10. 
PARATYPes. P.27053-001, 3 specimens, $12-18 \mathrm{~mm}$ SL, Raphael Island, Mauritius.

Exyrias akihito Allen and Randall, 2005b: 76, figures $1,2,3,4,5$.

Paratypes. P.31214.001, $67 \mathrm{~mm}$ SL, Madang Lagoon, PNG; P.32255.003, 2 specimens, 27-34 mm SL, Kawe Island, West Papua, Indonesia; P.32615.001, 81 mm SL, Milne Bay Province, PNG.

Feia nota Gill and Mooi, 1999: 366, figures 1, 2, 3a. HOLOTYPE. $P$ P.31440-001, 18-mm-SL,-Bessieres Island, WA, Australia.

Fusigobius duospilus Hoese and Reader, 1985: 2, figures $1,2$.

PARATYPes. P.25369-020， $29 \mathrm{~mm}$ SL, off Tantabiddi Creek, WA, Australia; P.25374-039, $38 \mathrm{~mm}$ SL, off Tantabiddi Creek, WA, Australia; P.25813-024, 3 specimens, 28-36 mm SL, South Muiron Island, WA, Australia; P.25819-025, 2 specimens, 35-42 mm SL, South Muiron Island, WA, Australia; P.26087-015, $31 \mathrm{~mm} \mathrm{SL}$, Christmas Island, Indian Ocean; P.26662-019, 2 specimens, 24-29 mm SL, Shark Bay, WA, Australia; P.26671-022, 2 specimens, $27-46 \mathrm{~mm}$ SL, Shark Bay, WA, Australia; P.27584-002, 44 mm SL, Houtman Abrolhos, WA, Australia; P.27587-016, $46 \mathrm{~mm}$ SL, Houtman Abrolhos, WA, Australia; P.27590-019, $40 \mathrm{~mm}$ SL, Houtman Abrolhos, WA, Australia.

REMARKS. The type description includes an error: P.27596-019 should read P.27590-019.

SYNONYMY. This species is now known as Coryphopterus duospilus.

Glossogobius coatesi Hoese and Allen, 1990: 118, figures 1, 2, 5a.

HoLOTYPE. P.29620-009, 97 mm SL, Ramu River, PNG.

PARATYPes. P.27847-009, 5 specimens, $24-79 \mathrm{~mm}$ SL, Kwatit River, PNG; P.29617-001, 3 specimens, $42-51 \mathrm{~mm}$ SL, Ramu River, PNG; P.29620-006, 9 specimens, 15-83 mm SL, Ramu River, PNG.

Glossogobius hoesei Allen and Boeseman, 1982: 84, figures 4, 5.

PARATYPES. P.27387-001, 10 specimens, 50-65 mm SL, Jow Lake, West Papua, Indonesia.

Glossogobius torrentis Hoese and Allen, 1990: 122, figures $3,4,5 b$.

HolotyPe. P.29615-009, 70 mm SL, Ramu River, PNG.

PaRATYPes. P.29614-007, 38 specimens, 15-56 mm SL; P.29615-002, 56 specimens, 17-71 mm SL; P.29619-006, 3 specimens, $26-44 \mathrm{~mm} \mathrm{SL}$, all from Ramu River, PNG.

ReMARKs. The type description includes two errors: paratypes P.29614-007 has 38 specimens, not 40, and paratypes P.29615-002 has 56 specimens, not 49 .

Lentipes dimetrodon Watson and Allen, 1999: 115, figure 2 .

Paratypes. P.31059-003, 4 specimens, 19-24 mm SL, Omamerwai Creek, West Papua, Indonesia.

REMARKS. The type description includes an error: P.31059-002 should read P.31059-003.

Lentipes venustus Allen, 2004d: 88, figures 1, 2.

HolotyPe. P.32355-001, $40 \mathrm{~mm}$ SL, Goodenough Island, PNG.

PARATYPes. P.32372-001, 2 specimens, 24-28 mm SL, Normanby Island, PNG; P.32374-001, 29 mm SL, Normanby Island, PNG.

Lentipes watsoni Allen, 1997b: 34, figures 1, 2.

HoLotyPe. P.31221-001, 47 mm SL, Sapoi River, PNG.

Paratypes. P.31221-002, 5 specimens, $45-64 \mathrm{~mm}$ SL, Sapoi River, PNG.

Mugilogobius filifer Larson, 2001: 119, figures 112, 113, 114, 115.

Paratypes. P.25668-014, 12 specimens, 17-32 mm SL, Admiralty Gulf, WA, Australia.

Mugilogobius littoralis Larson, 2001: 139, figures 133, 134, 135, 136.

PARATYPES. P.25668-018, $21 \mathrm{~mm}$ SL, Admiralty Gulf, WA, Australia; P.30303-017, 2 specimens, 13-21 mm SL, Napier Broome Bay, WA, Australia.

Myersina larsonae Allen, 1999d: 49, figure 1.

HolotyPe. P.31470-001, 47 mm SL, Cassini Island, WA, Australia.

Paratypes. P.31470-002, 2 specimens, $42-49 \mathrm{~mm}$ SL, Cassini Island, WA, Australia.

Papillogobius punctatus Gill and Miller, 1990: 509, figure 4 .

Holotype. P.29724-001, 51 mm SL, Swan River, WA, Australia.

Paratypes. P.29723-001, 5 specimens, 39-43 mm SL, Swan River, WA, Australia; P.29725-001, 39 mm SL, Swan River, WA, Australia; P.29726-001, 5 specimens, $41-53 \mathrm{~mm} \mathrm{SL}$, Swan River, WA, Australia; P.29727-001, 43 mm SL, Swan River, WA, Australia; P.29728-001, 2 specimens, 44-45 mm SL, Swan River, WA, Australia; P.29737-001, 6 specimens (all $\mathrm{c} / \mathrm{s}), 22-35 \mathrm{~mm}$ SL, Canning River, WA, Australia.

SYNONYMY. This species is now known as Favonigobius punctatus.

Periophthalmus murdyi Larson and Takita, 2004: 178 , figures $3,4,5,6$.

PARATYPes. P.32464-001, 4 specimens, $34-48 \mathrm{~mm}$ SL, Derby, WA, Australia. 
Pleurosicya coerulea Larson, 1990: 20, figure 16. Paratypes. P.28025-044, 36 specimens, 9-16 mm SL, Rowley Shoals, WA, Australia; P.29048-007, $11 \mathrm{~mm}$ SL, Ashmore Reef, WA, Australia.

Pleurosicya elongata Larson, 1990: 22, figure 17; Plate 2a.

Paratypes. P.30053-001, 2 specimens, $18-27 \mathrm{~mm}$ SL, Motupore Island, PNG.

Pleurosicya fringilla Larson, 1990: 25, figure 18. PARATYPES. P.27596-001, 2 specimens, 13-14 mm SL, Houtman Abrolhos, WA, Australia.

Pleurosicya plicata Larson, 1990: 41, figures 25, 26. Paratype. P.27666-027, 22 mm SL, Rowley Shoals, WA, Australia.

Priolepis fallacincta Winterbottom and Burridge, 1992: 1940, figures 5, 6 .

Paratype. P.27824-079, 15 mm SL, Manus, PNG.

Schismatogobius vitiensis Jenkins and Boseto, 2005: 76, figure 1.

Paratypes. P.32351-001, 5 specimens, 24-26 mm SL, Viti Levu, Fiji.

Sicyopus discordipinnis Watson, 1995: 273, figure 1. HoLOTYPE. P.27834-004, 25 mm SL, Letak Creek, PNG.

PARATYPes. P.27834-005, 2 specimens, 26-28 mm SL, Letak Creek, PNG; P.28167-001, 6 specimens, 29-34 mm SL, Arawa, PNG.

Sicyopus (Smilosicyopus) mystax Watson and Allen, 1999: 117, figure 3.

Paratypes. P.31262-004, 4 specimens, $30-40 \mathrm{~mm}$ SL, Aiyindor Creek, West Papua, Indonesia.

Signigobius biocellatus Hoese and Allen, 1977: 200, figures 1, 2, 3.

PARATYPES. P.24765-001, $48 \mathrm{~mm}$ SL, Lizard Island, Qld., Australia; P.25539-001, 24 mm SL, Koror Island, Palau.

Stenogobius (Insularigobius) alleni Watson, 1991: 632, figure 26.

HoLotyPE. P.28177-010, $36 \mathrm{~mm} \mathrm{SL}$, Tovarua Stream, PNG.

Paratypes. P.28177-003, 5 specimens, $28-39 \mathrm{~mm}$ SL, Tovarua Stream, PNG.

Stenogobius (Insularigobius) caudimaculosus Watson, 1991: 626, figure 23.

Paratypes. P.29317-001, 10 specimens, 39-66 mm SL, Marquesas Islands, Pacific Ocean.

Stenogobius (Insularigobius) hoesei Watson, 1991: 636, figure 28.

HoLOTYPE. P.27829-015, 60 mm SL, Manus Island, PNG.

PARATYPes. P.27829-017, 12 specimens, 23-47 mm
SL, Manus Island, PNG; P.27830-009, 42 mm SL, Manus Island, PNG; P.28163-003, 3 specimens, 28-34 mm SL, Bougainville, PNG; P.28164-007, 3 specimens, 26-50 mm SL, Bougainville, PNG; P.28185-015, 2 specimens, 36-42 $\mathrm{mm} \mathrm{SL,}$ Kavieng, PNG; P.28453-001, 2 specimens, 53-56 $\mathrm{mm} \mathrm{SL}$, Guadacanal, Solomon Islands.

Stenogobius (Stenogobius) lachneri Watson, 1994a: 162, figures $1,2,3,5$.

HoLotYPE. P.29973-010, 86 mm SL, Bintuni River, West Papua, Indonesia.

Paratypes. P.29981-004, 39 mm SL, Sorong, West Papua, Indonesia; P.29953-005, 2 specimens, 61$71 \mathrm{~mm}$ SL, Bintuni River, West Papua, Indonesia; P.29973-003, 15 specimens, 25-83 mm SL, Bintuni River, West Papua, Indonesia.

REMARKS. This name appeared first as Stenogobius lachneri Watson, 1990 in Allen (1991b) (p.198), but Watson did not publish his description until 1994. Eschmeyer et al. (2007) consider the name to be preoccupied due to the earlier publication by Allen and the authorship to be Watson in Allen, 1991b.

Stenogobius (Insularigobius) marqueti Watson, 1991: 622, figure 22.

PARATYPes. P.29656-001, 9 specimens, 36-60 mm SL, Marquesas Islands, Pacific Ocean.

Stenogobius (Stenogobius) psilosinionus Watson, 1991: 604, Fig 16.

Paratypes. P.28201-008, 20 specimens, 49-96 mm SL, Laloki River, PNG.

Stenogobius (Insularigobius) squamosus Watson, 1991: 629, figure 24.

Paratypes. P.29316-001, 9 specimens, 37-66 mm SL, Marquesas Islands, Pacific Ocean.

Stenogobius (Insularigobius) watsoni Allen, 2004d: 92, figures $6,7,8$.

Holotype. P.32368-001, 57 mm SL, Woodlark Island, PNG.

PARATYPES. P.32353-001, 3 specimens, $52-65 \mathrm{~mm}$ SL, Fergusson Island, PNG; P.32368-002, 2 specimens, 38-41 mm SL, Woodlark Island, PNG.

Stiphodon allen Watson, 1996: 114, figures 2, 4. HolotYPE. P.29644-001, 44 mm SL, Harvey Creek, Qld., Australia

Stiphodon atratus Watson, 1996: 115, figures 6, 8. PARATYPes. P.26413-001, 2 specimens, $29-31 \mathrm{~mm}$ SL, Beamu Creek, PNG; P.27788-004, 3 specimens, 25-32 mm SL, Oro Bay, PNG; P.27862-006, 2 specimens, 26-32 mm SL, Nabire River, West Papua, Indonesia; P.27862-007, 24 mm SL, Nabire River, West Papua, Indonesia; P.28185-012, 4 specimens, 22-42 mm SL, Kavieng, PNG; P.29613005, $28 \mathrm{~mm}$ SL, Bogia, PNG. 
Stiphodon birdsong Watson, 1996: 118, figures 11, 13.

HoLOTYPe. P.27834-007, 23 mm SL, Letak Creek, PNG.

Paratypes. P.31059-001, 3 specimens, $18-21 \mathrm{~mm}$ SL, Omamerwai Creek, West Papua, Indonesia; P.31059-002, 2 specimens, 20-22 mm SL, Omamerwai Creek, West Papua, Indonesia.

REMARKS. The type description includes an error: for the paratypes P.31059-002 there are 2 specimens, not 5 .

Stiphodon larson Watson, 1996: 120, figures 14, 16. HoLOTYPE. P.29602-017, $28 \mathrm{~mm}$ SL, Maeabudu River, PNG.

PARATYPes. P.29600-004, 2 specimens, 26-28 mm SL; P.29601-007, 4 specimens, 19-26 mm SL; P.29602-013, 50 specimens, $21-31 \mathrm{~mm} \mathrm{SL}$, all from Gogol, PNG.

REMARKS. The type description includes an error: for the paratypes P.29602-013 there are 50 specimens, not 51 .

Stiphodon rutilaureus Watson, 1996: 122, figure 18, 20.

HoLotyPe. P.27834-003, 24 mm SL, Letak Creek, PNG.

PARATYPes. P.27833-014, 2 specimens, $15-20 \mathrm{~mm}$ SL, Mandi Stream, PNG; P.28164-008, 16 specimens, $18-30 \mathrm{~mm}$ SL, Tekan River, PNG; P.28164-009, 2 specimens, 21-23 mm SL, Tekan River, PNG; P.28423-003, 10 specimens, 23-29 $\mathrm{mm} \mathrm{SL}$, Efate Island, Vanuatu.

Stiphodon weberi Watson, Allen and Kottelat, 1998: 299, figures 5, 8 .

PaRATYPES. P.31037-006, $35 \mathrm{~mm}$ SL, Reifafeif River, West Papua, Indonesia; P.31038-002, 2 specimens, 24-25 mm SL, Tare River, West Papua, Indonesia.

Sueviota atrinasa Winterbottom and Hoese, 1988: 7, figure 5.

PARATYPes. P.28022-054, 3 specimens, $17-20 \mathrm{~mm}$ SL, Rowley Shoals, WA, Australia; P.28031-036, $15 \mathrm{~mm}$ SL, Rowley Shoals, WA, Australia.

Trimma dalerocheila Winterbottom, 1984: 697, figures 1,11 .

PARATYPES. P.27983-001, 5 specimens, $16-17 \mathrm{~mm}$ SL, Chagos Archipelago, Indian Ocean.

Trimma emeryi Winterbottom, 1985: 752, figure 5. PARATYPES. P.28333-001, 14 specimens, $10-18 \mathrm{~mm}$ SL, Chagos Archipelago, Indian Ocean; P.28334001, 7 specimens, 13-15 mm SL, Chagos Archipelago, Indian Ocean.

Trimma haima Winterbottom, 1984: 702, figures 3,17 .
Paratypes. P.28015-001, 9 specimens, $10-16 \mathrm{~mm}$ SL, Chagos Archipelago, Indian Ocean.

Trimma halonevum Winterbottom, 2000: 62, Fig + figure 3.

Paratypes. P.28176-007, $18 \mathrm{~mm}$ SL, Rabaul, PNG; P.28180-014, 3 specimens, 20-21 mm SL, Rabaul, PNG.

Trimma lantana Winterbottom and Villa, 2003: 16, figures $1 b, 2,3$.

PaRATYPES. P.27827-046, 13 mm SL, Manus, PNG; P.27970-037, 5 specimens, $22-27 \mathrm{~mm}$ SL, Point Quobba, WA, Australia; P.29047-034, 5 specimens, 19-23 mm SL, Ashmore Reef, WA, Australia; P.29051-024, 2 specimens, $19-26 \mathrm{~mm}$ SL, Ashmore Reef, WA, Australia.

REMARKS. The type description includes an error: P.27920-037 should read P.27970-037.

Trimma rubromaculata Allen and Munday, 1995: 102 , figures 5,6 .

HolotyPe. P.30889-001, 18 mm SL, Kimbe Bay, PNG.

Paratypes. P.30889-002, 2 specimens, 14-16 mm SL, Kimbe Bay, PNG.

Trimma stobbsi Winterbottom, 2001: 20, figures 1, 2.

Paratypes. P.28170-002, 5 specimens, $15-16 \mathrm{~mm}$ SL, Rabaul, PNG.

Tryssogobius colini Larson and Hoese, 2001: 29, figures 1, 2, 3, 4, 5, 6 .

Paratype. P.27826-094, $21 \mathrm{~mm}$ SL, Los Negros Island, PNG.

REMARKS. The type description includes an error: P.27826-[ex006] should read P.27826-094.

Valenciennea alleni Hoese and Larson, 1994: 13, Plates 1a, 5a.

Paratypes. P.25168-001, $74 \mathrm{~mm}$ SL, Shark Bay, WA, Australia; P.26667-008, 37 mm SL, Shark Bay, WA, Australia.

Valenciennea decora Hoese and Larson, 1994: 18, Plates 1c, d, $5 b$.

Paratype. P.27974-001, $72 \mathrm{~mm}$ SL, New Caledonia, Pacific Ocean.

Valenciennea parva Hoese and Larson, 1994: 37, Plates 3a, 5 h.

Paratype. P.27973-001, $40 \mathrm{~mm}$ SL, Palfrey Island, Qld., Australia

Vanderhorstia flavilineata Allen and Munday, 1995: 103 , figures $7,8$.

HolotyPe. P.30896-001, 27 mm SL, Kimbe Island, PNG.

Paratype. P.30896-003, 22 mm SL, Kimbe Island, PNG. 
Vanderhorstia nobilis Allen and Randall, 2006: 40, figures $1,2,3,4$.

HoLotype. P.31402-002, 43 mm SL, Culion Island, Philippines.

\section{Eleotrididae}

Allomogurnda flavimarginata Allen, 2003: 981, figure 5 .

Holotype. P. 32377-001, 77 mm SL, Bulolu, PNG. Paratypes. P.27407-001, 2 specimens, 51-61 mm SL, Bulolu, PNG; P. 32377-002, 4 specimens, 34$64 \mathrm{~mm}$ SL, Bulolu, PNG.

Allomogurnda insularis Allen, 2003: 984, figures 7, 8,9 .

Holotype. P.32356-001, $55 \mathrm{~mm}$ SL, Matalamoia River, PNG.

Paratypes. P.32356-002, 10 specimens, $28-38 \mathrm{~mm}$ SL, Matalamoia River, PNG.

Allomogurnda landfordi Allen, 2003: 986, figure 11. HolotyPe. P.30969-001, 37 mm SL, Kikori, PNG.

PARATYPes. P.30969-002, 10 specimens, 16-33 mm SL, Kikori, PNG; P.32378-001, 58 mm SL, Aseki, PNG.

Allomogurnda montana Allen, 2003: 988, figure 13. HolotYPE. P.32370-001, $61 \mathrm{~mm} \mathrm{SL}$, Awaetowa River, PNG.

ParatYPes. P.32370-002, 6 specimens, 18-54 mm SL, Awaetowa River, PNG.

Allomogurnda papua Allen, 2003: 992, figures 19, 20.

HoLOTYPE. P.27397-002, 66 mm SL, Matairuka Village, PNG.

PARATYPes. P.26414-001, 10 specimens, 17-65 mm SL, Banderi, PNG; P.27783-003, 18 specimens, 14-81 mm SL, Foasi Creek, PNG; P.27784-005, 14 specimens, 17-57 mm SL, Moni River, PNG; P.27787-001, 2 specimens, 47-62 mm SL, Oro Bay, PNG; P.27791-004, 3 specimens, 39-65 mm SL, Popondetta, PNG.

REMARKS. The type description includes an error: paratypes P.27783-002 should read P.27783-003.

Allomogurnda sampricei Allen, 2003: 995, figures 22, 23.

PaRATYPes. P.32375-001, 3 specimens, 30-43 mm SL, Reifafeif River, Indonesia; P32376-001, 32 mm SL, Reifafeif River, Indonesia.

Eleotris aquadulcis Allen and Coates, 1990: 133, figures 1, 2.

HoLotYPE. P.29608-006, 205 mm SL, Twenty Mile Island Lake, PNG.

Paratypes. P.28206-007, 9 specimens, 61-183 mm SL, Sepik River, PNG; P.29608-002, 5 specimens, $160-215 \mathrm{~mm}$ SL, Twenty Mile Island Lake, PNG.
Eleotris aurea Shipway, 1950: 75, figures 1, 2. Holotype. P.3283-001, 59 mm SL, Murchison River, WA, Australia.

SYNONYMY. This species is now known as Hypseleotris aurea.

Hypseleotris ejuncida Hoese and Allen in Allen, 1982d: 56 , figure 6 .

HoLOTYPe. P.25032-009, 46 mm SL, Gundarara Creek, near Prince Regent River, WA, Australia.

Paratypes. P.25032-008, 8 specimens, $23-43 \mathrm{~mm}$

SL, Gundarara Creek, WA, Australia.

REMARKS. Hoese and Allen (1983) provided a full description of this species, but prior to its publication, a brief account and illustration of the species was published by Allen (1982d), attributing the name of the species to the two authors. Although the diagnosis in the latter account is not sufficiently detailed to distinguish the new species from other Hypseleotris, a combination of the diagnosis, the illustration and the unique location of collection identifies the species. The authority of the species is therefore Hoese and Allen in Allen (1982d) (Eschmeyer 2007). The description of the species by Hoese and Allen (1983) includes an error: paratypes P.25032-002 should read P.25032-008.

Hypseleotris kimberleyensis Hoese and Allen in Allen, 1982d: 57, Plate 13.

HolOTYPE. P.25454-009, 34 mm SL, Barnett River, WA, Australia.

Paratypes. P.25454-007, 6 specimens, $27-34 \mathrm{~mm}$ SL, Barnett River, WA, Australia; P.25872-006, 4 specimens, 28-34 mm SL, Manning Creek, WA, Australia.

REMARKS. This name was first published as Hypseleotris kimberleyensis Hoese and Allen in Allen (1982d) (see above account of Hypseleotris ejuncida).

Hypseleotris regalis Hoese and Allen in Allen, 1982d: 57, Plate 13.

Holotype. P.25028-009, $32 \mathrm{~mm}$ SL, Wyulda Creek, near Roe River, WA, Australia.

PARATyPes. P.25028-007, 11 specimens, 23-36 mm SL, Wyulda Creek, WA, Australia; P.25040-004, 14 specimens, 22-37 mm SL, Youwanjela Creek, WA, Australia.

REMARKS. This name was first published as Hypseleotris regalis Hoese and Allen in Allen (1982d) (see above account of Hypseleotris ejuncida). The description of the species by Hoese and Allen (1983) includes an error: for the paratypes P.25028-007, there are 11 specimens, not 12.

Kimberleyeleotris hutchinsi Hoese and Allen, 1987: 36 , figures $1,2$.

Holotype. P.25684-009, $31 \mathrm{~mm}$ SL, Mitchell River, WA, Australia. 
PARATYPES. P.25683-008, 2 specimens, 22-24 mm SL, Mitchell River, WA, Australia; P.25684-007, 14 specimens, 25-30 mm SL, Mitchell River, WA, Australia.

Kimberleyeleotris notata Hoese and Allen, 1987: 40, figures 3,4 .

Holotype. P.25427-010, $37 \mathrm{~mm}$ SL, Drysdale River, WA, Australia.

Milyeringa veritas Whitley, 1945: 36, figure 15.

HoLotYPE-P.2913-001,40-mm SL, North West Cape, WA, Australia.

Mogurnda aiwasoensis Allen and Renyaan, 1996c: 52, figure 11.

Paratype. P.31042-005, 38 mm SL, Lake Aiwaso, West Pápua, Indonesia.

Mogurnda cingulata Allen and Hoese, 1991: 36, figure 3 .

Holotype. P.27806-003, $94 \mathrm{~mm}$ SL, tributary of Ok Tedi River, PNG.

PARATYPES. P.27388-002, 35 specimens, 32-115 mm SL, Digul River, Indonesia; P.27793-004, 2 specimens, 20-50 mm SL, Kiunga, PNG; P.27794003, $46 \mathrm{~mm}$ SL, Kiunga, PNG; P.27799-008, 2 specimens, 29-36 mm SL, Ningerum, PNG; P.27807-003, $57 \mathrm{~mm}$ SL, Kiunga, PNG; P.27808005, 5 specimens, 35-65 mm SL, Kiunga, PNG; P.27812-007, $81 \mathrm{~mm}$ SL, tributary of Nomad River, $\mathrm{PNG}$.

Mogurnda furva Allen and Hoese, 1986: 83, figures 3,4 .

Holotype. P.28158-009, 65 mm SL, Lake Kutubu, PNG.

PARATyPes. P.28158-011, 2 specimens, 19-31 mm SL, Lake Kutubu, PNG.

Mogurnda kaifayama Allen and Jenkins, 1999a: 244, figures 8, 9, 10, 11.

PARATYPEs. P.31301-003, 16 specimens, 23-89 mm SL, Lake Kaifayama, Indonesia.

Mogurnda kutubuensis Allen and Hoese, 1986: 86, figures 6,7 .

Holotype. P.28159-011, 87 mm SL, Lake Kutubu, PNG.

Paratypes. P.28158-008, 30 specimens, 21-68 mm SL, Lake Kutubu, PNG; P.28159-008, 113 specimens, 16-77 mm SL, Lake Kutubu, PNG.

REMARKS. The type description includes an error: for the paratypes P. 28159-008 there are 113 specimens, not 112 .

Mogurnda lineata Allen and Hoese, 1991: 39, figure 4.

Holotype. P.27790-008, 81 mm SL, Kaili Creek, PNG.

PARATYPES. P.27790-005, 21 specimens, 22-82 mm SL, Kaili Creek, PNG.
Mogurnda maccuneae Jenkins, Buston and Allen, 2000: 48, figures 2, 3 .

HolotyPe. P.31430-001, 73 mm SL, Lake Kutubu, PNG.

Paratypes. P.31430-002, 7 specimens, 33-74 mm SL, Lake Kutubu, PNG.

Mogurnda magna Allen and Renyaan, 1996c: 49, figures 5, 6.

PARATYPES. P.31042-001, 14 specimens, 22-86 mm SL, Lake Aiwaso, West Papua, Indonesia; -P.31043-002,-10-specimens, 23-80 mm_SL, Lake Laamora, West Papua, Indonesia.

Mogurnda malsmithi Allen and Jebb, 1993: 238, figure 5 .

Holotype. P.30381-005, 56 mm SL, Pio, PNG.

PARATYPes. P.30348-003, 2 specimens, 35-68 mm SL, Koma River, PNG.

Mogurnda mbuta Allen and Jenkins, 1999a: 239, figures 4, 5 .

Paratypes. P.31300-005, 20 specimens, 29-92 mm SL, Lake Mbutu, West Papua, Indonesia.

Mogurnda mosa Jenkins, Buston and Allen, 2000: 52, figures 7, 8.

Holotype. P.31431-001, $117 \mathrm{~mm}$ SL, Lake Kutubu, PNG.

PARATYPes. P.31431-002, 3 specimens, 84-108 mm SL, Lake Kutubu, PNG.

Mogurnda oligolepis Allen and Jenkins, 1999b: 151, figure 9.

Holotype. P.25405-012, 59 mm SL, Morgunda Creek, WA, Australia.

Paratypes. P.25024-004, 10 specimens, 26-36 mm SL, Charnley River, WA, Australia; P.25028-008, $36 \mathrm{~mm}$ SL, Wyulda Creek, WA, Australia; P.25031-003, 2 specimens, 43-49 mm SL, Prince Regent River, WA, Australia; P.25034-002, 4 specimens, 32-39 mm SL, Cascade Creek, WA, Australia; P.25041-002, 2 specimens, $40-44 \mathrm{~mm}$ SL, Blyxa Creek, WA, Australia; P.25405-009, 12 specimens, 26-55 mm SL, Mogurnda Creek, WA, Australia; P.25406-007, 3 specimens, $27-40$ mm SL, Mogurnda Creek, WA, Australia; P.25408004, 4 specimens, 22-55 mm SL, Mogurnda Creek, WA, Australia; P.25422-003, 6 specimens, 39-51 mm SL, Palmoondoora Creek, WA, Australia; P.25426-002, 4 specimens, 22-36 mm SL, Forest Creek, WA, Australia; P.25428005, 10 specimens, 22-58 mm SL, Berkeley River, WA, Australia; P.25455-003, 5 specimens, 27-56 mm SL, Kennedy Creek, WA, Australia; P.25458-006, 4 specimens, 37-56 $\mathrm{mm}$ SL, Crossland Creek, WA, Australia; P.25463-003, 9 specimens, 16-63 mm SL, Carson River, WA, Australia.

REMARKS. The type description includes an error: paratypes P.25422-00 should read P.25422-003. 
Mogurnda orientalis Allen and Hoese, 1991: 41, figure 5 .

HoLotrPe. P.27785-004, 102 mm SL, Safia Creek, PNG.

Paratypes. P.27784-004, 3 specimens, $63-70 \mathrm{~mm}$ SL, Moni River, PNG.

Mogurnda pardalis Allen and Renyaan, 1996c: 51, figures 8,9 .

PARATYPes. P.30519-003, 3 specimens, 56-127 mm SL, Lake Kamakawala, West Papua, Indonesia.

Mogurnda spilota Allen and Hoese, 1986: 90, figures 8,9 .

HoLOTYPE. P.28159-010, 90 mm SL, Lake Kutubu, PNG.

Paratype. P.28158-010, 56 mm SL, Lake Kutubu, PNG.

Mogurnda vitta Allen and Hoese, 1986: 95, figures $12,13$.

Holotype. P.28159-012, 104 mm SL, Lake Kutubu, PNG.

PARATYPE. P.28159-013, 55 mm SL, Lake Kutubu, PNG.

Mogurnda wapoga Allen, Jenkins and Renyaan, 1999: 64, figure 2.

PARATYPeS. P.31449-002, 5 specimens, $41-55 \mathrm{~mm}$ SL, Tiawiwa River Floodplain, West Papua, Indonesia.

Oxyeleotris altipinna Allen and Renyaan, 1996c: 53, figure 13.

Paratypes. P.31042-002, 5 specimens, 19-38 mm SL, Lake Aiwaso, West Papua, Indonesia.

Oxyeleotris caeca Allen, 1996b: 44, figures 1, 2. HoLOTYPE. P.31011-001, 107 mm SL, Kikori, PNG.

Oxyeleotris stagnicola Allen, Hortle and Renyaan, 2000: 171, figures (p.149).

Paratype. P.31258-011, 38 mm SL, Timika, West Papua, Indonesia.

Oxyeleotris wisselensis Allen and Boeseman, 1982: 90, figures 6, 7.

PARATYPes. P.27389-001, 17 specimens, 26-82 mm SL, Tage Lake, West Papua, Indonesia.

Thalasseleotris adela Hoese and Larson, 1987: 45, figures 1, 2, 3, 4 .

PARATYPES. P.25770-023, $24 \mathrm{~mm}$ SL, Recherche Archipelago, WA, Australia; P.26005-011, $20 \mathrm{~mm}$ SL, Recherche Archipelago, WA, Australia; P.26060-016, 2 specimens, 16-20 mm SL, Rottnest Island, WA, Australia; P.26616-025, 2 specimens, 24-24 mm SL, Rottnest Island, WA, Australia; P.26620-023, 3 specimens, 15-20 mm SL, Rottnest Island, WA, Australia; P.27136-015, 3 specimens, 21-25 mm SL, Victor Harbour, SA, Australia; P.27542-004, $18 \mathrm{~mm}$ SL, Port Davey, Tas.,
Australia; P.27543-015, $30 \mathrm{~mm}$ SL, Port Davey, Tas., Australia; P.27544-014, 4 specimens, 21-22 mm SL, Port Esperance, Tas., Australia; P.27546012, 8 specimens, 13 $22 \mathrm{~mm}$ SL, Esperance Point, Tas., Australia; P.27547-009, 6 specimens, 22-25 mm SL, Port Esperance, Tas., Australia; P.27549015, 3 specimens, $22-23 \mathrm{~mm} \mathrm{SL}$, Port Arthur, Tas., Australia; P.27554-018, 4 specimens, 19-22 mm SL, Spring Bay, Tas., Australia; P.27555-011, 4 specimens, $19-20 \mathrm{~mm}$ SL, Saint Helens, Tas., Australia; P.27564-014, 4 specimens, $13-21 \mathrm{~mm}$ SL, Bridport, Tas., Australia; P.27616-033, $20 \mathrm{~mm}$ SL, Rottnest Island, WA, Australia; P.28293-033, 9 specimens, 15-22 mm SL, Lucky Bay, WA, Australia; P.28296-047, 6 specimens, 19-25 mm SL, Recherche Archipelago, WA, Australia; P.28297-026, 2 specimens, 12-22 mm SL, Lucky Bay, WA, Australia; P.28298-017, 21 mm SL, Lucky Bay, WA, Australia; P.28300-028, 3 specimens, 12-23 mm SL, Lucky Bay, WA, Australia.

REMARKS. The type description includes several errors: P.27543-005 should read P.27543-015; P.27547-007 should read P.27547-009; P.25570022 should read P.25770-023 and P.28297-025 should read P.28297-026.

\section{Xenisthmidae}

Xenisthmus chi Gill and Hoese, 2004: 242, figure 1. Holotype. P.28030-033, $21 \mathrm{~mm}$ SL, Rowley Shoals, WA, Australia.

PARATYPe. P.28030-041, $22 \mathrm{~mm}$ SL, Rowley Shoals, WA, Australia.

Xenisthmus semicinctis Gill and Hoese, 2004: 245, figure 3.

Holotype. P.28025-048, 19 mm SL, Rowley Shoals, WA, Australia.

Paratype. P.28025-068, $18 \mathrm{~mm}$ SL, Rowley Shoals, WA, Australia.

\section{Gobioididae}

Gymnoamblyopus novaeguineae Murdy and Ferraris, 2003: 2, figure 1.

HoLotype. P.29823-001, 117 mm SL, Fly River, PNG.

\section{Microdesmidae}

Aioliops tetrophthalmus Rennis and Hoese, 1987: 79, figures 1, 15.

PARATYPES. P.28454-001, 2 specimens, 15-20 mm SL, Lizard Island, Qld (missing), Australia.

Parioglossus marginalis Rennis and Hoese, 1985: 179, figures 9, 23.

PARATYPES. P.28011-001, 3 specimens, 32-33 mm SL, Point Hacking, NSW, Australia. 
Pholidichthys anguis Springer and Larson, 1996:

354, figures 1, 2, 3 .

Paratype. P.31012-001, 3 specimens, Arafura Sea, NT, Australia.

Ptereleotris monoptera Randall and Hoese, 1985: 24, Plate 3 (figures c, d); figure 8.

Paratypes. P.25236-017, $58 \mathrm{~mm} \mathrm{SL}$, Ambon Island, Indonesia; P.27077-002, $74 \mathrm{~mm} \mathrm{SL}$, Solitary Islands, NSW, Australia; P.28449-001, 84 mm SL, Society Islands, Pacific Ocean.

REMARKS. The type description includes an error: the measurement for paratype P.25236-017 is given as $580 \mathrm{~mm} \mathrm{SL}$, whereas it should read 58 $\mathrm{mm}$ SL.

\section{Acanthuridae}

Acanthurus albipectoralis Allen and Ayling, 1987: 15, figure 1.

Paratype. P.28537-001, 261 mm SL, Herald Cays, Qld, Australia

Ctenochaetus cyanocheilus Randall and Clements, 2001: 9, Plate 1b, 4c, d, e, f.

PARATYPES. P.27980-019, $155 \mathrm{~mm}$ SL, Monte Bello Islands, WA, Australia; P.31434-028, $67 \mathrm{~mm} \mathrm{SL}$, Ashmore Reef, WA, Australia.

Ctenochaetus truncatus Randall and Clements, 2001: 25, Plates 3c, 6f, g, h.

Paratype. P.25010-001, $152 \mathrm{~mm}$ SL, Christmas Island, Indian Ocean.

\section{Siganidae}

Siganus trispilos Woodland and Allen, 1977: 617, figure 1.

HoLOTYPE. P.25366-001, $155 \mathrm{~mm}$ SL, North West Cape, WA, Australia.

PARATYPE. P.25542-003, $152 \mathrm{~mm} \mathrm{SL}$, North West Cape, WA, Australia.

\section{Trichiuridae}

Evoxymetopon anzac Alexander, 1917: 104, Plate 7. Holotype. P.352-001, $1415 \mathrm{~mm}$ SL, North Fremantle, WA, Australia.

SynONYMY. This species is now known as Assurger anzac.

\section{Scombridae}

Rastrelliger serventyi Whitley, 1944a: 268.

HoLotype. P.181-001, 270 mm SL, Port Hedland, WA, Australia.

REMARKS. The type description includes an error: P.18 should read P.181.

SYNONYMY. This name is a junior synonym of Rastrelliger kanagurta (Cuvier, 1816).

\section{Bothidae}

Arnoglossus micrommatus Amaoka, Arai and Gomon, 1997: 131, figure 1.

Paratypes. P.28613-009, 2 specimens, 87-98 mm SL, King George Sound, WA, Australia.

Asterorhombus osculus Amaoka and Arai, 1998: 254, figure 4.

PaRATYPES. P.25396-024, 5 specimens, 84-92 mm SL, Rowley Shoals, WA, Australia.

REMARKS. The type description includes an error: P.25396-010 should read P.25396-024.

SYNONYMY. This species is now known as Engyprosopon osculus.

\section{Soleidae}

Aseraggodes crypticus Randall and Allen, 2007: 110 , figure 1 .

HoLotype, P.28990-020, 36 mm SL, Christmas Island, Indian Ocean.

PARATYPE. P.26103-016, $49 \mathrm{~mm}$ SL, Christmas Island, Indian Ocean.

REMARKS. The type description includes errors: the collection date for the holotype should read 20 June 1986 and paratypes P.26108-016 should read P.26103-016.

Leptachirus alleni Randall, 2007: 85, figure 2.

HolotyPe. P.29959-004, 71 mm SL, Bintuni River, West Papua, Indonesia.

Paratypes. P.29959-015, 6 specimens, 57-82 mm SL, Bintuni River, West Papua, Indonesia.

Leptachirus bensbach Randall, 2007: 88, figure 3.

Holotype. P.27820-003, 69 mm SL, Bensbach River, Indonesia.

PARATYPES. P.27820-007, 67 mm SL; P.27822-004, 2 specimens, 76-96 mm SL; P.31342-004, $108 \mathrm{~mm}$ SL, all from Bensbach River, Indonesia.

RemarKs. The type description includes an error: paratypes P.31342-001 should read P.31342-004.

Leptachirus kikori Randall, 2007: 93, figure 5.

Holotype. P.31210-002, 72 mm SL, Kikori River, PNG.

PARATYPES. P.30977-020, 13 specimens, 24-58 mm SL, Kikori River, PNG; P.31210-018, 6 specimens, 27-43 mm SL, Kikori River, PNG.

Leptachirus lorentz Randall, 2007: 96, figure 7. PARATYPE. P.32818-001, 74 mm SL, Lorentz River, West Papua, Indonesia.

Leptachirus polylepis Randall, 2007: 99, figure 8. ParatYPes. P.32819-001, 2 specimens, 51-58 mm SL, East Alligator River, NT, Australia; P.25895002, $54 \mathrm{~mm}$ SL, South Alligator River, NT, Australia. 
Leptachirus robertsi Randall, 2007: 102, figure 9. Paratypes. P.28150-010, $34 \mathrm{~mm}$ SL, Lower Fly River, PNG; P.28151-006, 3 specimens, 22-34 mm SL, Lower Fly River, PNG.

Leptachirus triramus Randall, 2007: 104, figure 10. PARATYPES. P.20608-001, 4 specimens, 42-59 mm SL, Ord River, WA, Australia; P.30067-007, 4 specimens, 44-52 mm SL, Ord River, WA, Australia.

Soleichthys serpenpellis Munroe and Menke, 2004: 253, figures 2, 3.

Paratype. P. 5739-001, 100 mm SL, Dampier Archipelago, WA, Australia.

Synaptura hediste Mees, 1964b: 49, Plate 4.

HoLotYPE. P.5532-001, 126 mm SL, Cheyne Head, WA, Australia.

Paratypes. P.5533-001, 117 mm SL, Albany, WA, Australia; P.5534-001, $126 \mathrm{~mm}$ SL, St Vincent Gulf, SA, Australia.

\section{Cynoglossidae}

Cynoglossus broadhursti Waite, 1905: 73, Plate 8 (figure 2).

SYNTYPES. P.13258-001, $255 \mathrm{~mm}$ SL, between Fremantle and Geraldton, WA, Australia.

REMARKS. Waite's description was based on five specimens sent to AMS from WAM (see section on Waite's types in Introduction). Three of these were retained at AMS (AMS I.7232 to I.7234) and the remaining two were returned to WAM. Only one of the latter, P.13258-001, could be located at WAM and the other is missing, presumed lost. The type locality given as "Carnarvon to the northward of Houtman's Abrolhos" was disputed by McKay (1965), who believed it to be Mandurah. However, the first registration of this syntype ("6814", in the Taxidermists Book) gives the locality as "Between Fremantle and Geraldton". Mandurah is mentioned in a subsequent entry in the Catalogue of the Museum (registration number "157"). In 1965, McKay reregistered the specimen as P.13258, recording Mandurah as the collection locality, apparently unaware of the earlier entry in the Taxidermists Book. As the syntype still has the original label attached to it with "6814" clearly visible, we recognise the region between Fremantle and Geraldton as the collection locality.

Paraplagusia Iongirostris Chapleau, Renaud and Kailola, 1991: 240, figure 1.

Holotype. P.29719-001, 206 mm SL, Joseph Bonaparte Gulf, WA, Australia.

Paratypes. P.29719-002, 5 specimens (1 c/s), 202$243 \mathrm{~mm}$ SL, Joseph Bonaparte Gulf, WA,
Australia; P.29753-001, 7 specimens (1 c/s), 94$217 \mathrm{~mm}$ SL, Joseph Bonaparte Gulf, WA, Australia.

REMARKS. The type description includes an error: paratypes P.29719-001 should read P.29719-002.

Paraplagusia sinerama Chapleau and Renaud, 1993: 799, figure 1a.

Paratypes. P.29719-004, 2 specimens, 173-207 mm SL, Joseph Bonaparte Gulf, WA, Australia. REMARKS. The type description includes an error: paratypes P.29719-001 should read P.29719-004.

\section{Monacanthidae}

Chaetodermis maccullochi Waite, 1905: 81, Plate 17. HoLOTYPE. P.13260-001, 230 mm SL, Houtman Abrolhos, WA, Australia.

SYNONYMY. This name is a junior synonym of Chaetodermis penicilligerus (Cuvier, 1816).

Colurodontis paxmani Hutchins, 1977: 22, figure 7. HolotyPE. P.15454-001, 120 mm SL, Shark Bay, WA, Australia.

Paratypes. P.14871-001, 78 mm SL, Shark Bay, WA, Australia; P.15455-001, 118 mm SL, Shark Bay, WA, Australia; P.23242-001 to P.23252-001, 11 specimens, 12-16 mm SL, Monte Bello Islands, WA, Australia; P.24124-001 to P.24125001, 2 specimens, 11-12 mm SL, Kendrew Island, WA, Australia; P.24126-001 to P.24127-001, 2 specimens, 13-14 mm SL, Kendrew Island, WA, Australia; P.25568-001, 2 specimens, 68-79 mm SL, Shark Bay, WA, Australia; P.25569-001, 8 specimens, 14-17 mm SL, Exmouth Gulf, WA, Australia; P.25570-001 (skin and skel.), $74 \mathrm{~mm}$ SL, Shark Bay, WA, Australia; P.25571-001, 42 mm SL, Shark Bay, WA, Australia; P.25572-001, $31 \mathrm{~mm}$ SL, Onslow, WA, Australia; P.25573-001, $87 \mathrm{~mm}$ SL, Woodman's Point, WA, Australia.

Eubalichthys caeruleoguttatus Hutchins, 1977: 28, figure 8.

HoLOTYPE. P.5204-001, 212 mm SL, Beagle Island, WA, Australia.

PARATYPes. P.9156-001, 129 mm SL, Shark Bay, WA, Australia; P.9157-001, $134 \mathrm{~mm}$ SL, Shark Bay, WA, Australia; P.9159-001, 115 mm SL, Exmouth Gulf, WA, Australia; P.11828-001 to P.11829-001, 2 specimens, 117-142 mm SL, Shark Bay, WA, Australia; 14845-001, $190 \mathrm{~mm} \mathrm{SL}$ (skel.), Shark Bay, WA, Australia; P.14875-001, $115 \mathrm{~mm}$ SL, Exmouth Gulf, WA, Australia; P.14887-001, $100 \mathrm{~mm}$ SL, Exmouth Gulf, WA, Australia; P.22100-001, 253 mm SL, Cape Cuvier, WA, Australia; P.22310-001, $241 \mathrm{~mm} \mathrm{SL,} \mathrm{Cape}$ Cuvier, WA, Australia; P.25555-001, 35 mm SL, Houtman Abrolhos, WA, Australia; P.25556-001, $53 \mathrm{~mm}$ SL, Shark Bay, WA, Australia; P.25557001, $131 \mathrm{~mm} \mathrm{SL}$, Carnarvon, WA, Australia; 
P.25558-001, 5 specimens, 76-98 mm SL, Point Quobba, WA, Australia; P.25559-001, 44 mm SL, Shark Bay, WA, Australia; P.25579-001, 245 mm SL, Shark Bay, WA, Australia.

REMARKS. The type description includes an error: for the paratypes P.25558-001 there are 5 specimens, not 6 .

Eubalichthys cyanoura Hutchins, 1987b: 51, figure 1.

HөLОTYPE-P.25762-001, 232 mm SL, Recherche Archipelago, WA, Australia.

PARATYPeS. P.24841-001, 159 mm SL, Albany, WA, Australia; P.25998-001, 104 mm SL, Garden Island, WA, Australia; P.27137-001, 2 specimens, 138-238 mm SL, Yorke Peninsula, SA, Australia; P.28393-004, 156 mm SL, Geographe Bay, WA, Australia; P.28865-002, 299 mm SL, Safety Bay, WA, Australia.

Eubalichthys fuscosinus Hutchins, 1977: 33, figure 9.

Holotype. P.25580-001, 273 mm SL, Koks Island, WA, Australia.

PARATYPes. P.25579-002, 2 specimens, 231-253 mm SL

SYNONYMY. This name is a junior synonym of Eubalichthys caeruleoguttatus (Hutchins, 1977).

Lalmohania velutina Hutchins, 1994: 570, figure 2.

Paratype. P.30671-001 (c/s and skin), $55 \mathrm{~mm} \mathrm{SL}$, Kilakkari, India.

Meuschenia flavolineata Hutchins, 1977: 40, figure 11.

HolotyPe. P.25489-001, 233 mm SL, Rottnest Island, WA, Australia.

Paratypes. P.9155-001, 144 mm SL, Irwin Point, WA, Australia; P.24514-001, $157 \mathrm{~mm} \mathrm{SL}$, Canal Rocks, WA, Australia; P.25150-008, 137 mm SL, Yallingup, WA, Australia; P.25173-001, $162 \mathrm{~mm}$ SL (skin and skel.), Rottnest Island, WA, Australia; P.25250-004, 176 mm SL, Rottnest Island, WA, Australia; P.25251-003, 2 specimens, 178-190 mm SL, Rottnest Island, WA, Australia; P.25252-002, $201 \mathrm{~mm}$ SL, Carnac Island, WA, Australia; P.25549-001, $81 \mathrm{~mm} \mathrm{SL}$, Woodman's Point, WA, Australia; P.25550-001, $48 \mathrm{~mm}$ SL, Augusta, WA, Australia; P.25551001, $67 \mathrm{~mm}$ SL, Rottnest Island, WA, Australia; P.25553-001, $239 \mathrm{~mm}$ SL, Rottnest Island, WA, Australia.

Meuschenia venusta Hutchins, 1977: 45, figure 12. HoLOTYPE. P.14881-001, 110 mm SL, Shark Bay, WA, Australia.

Paramonacanthus arabicus Hutchins, 1997: 9, figures 8,9 .

HoLoTYPE. P.31180-001, 67 mm SL, Arabian Gulf, Saudi Arabia.
ParatyPes. P.25977-010, 11 specimens, 33-69 mm SL, Bahrain; P.25979-003, 3 specimens, 50-68 mm SL, Qatar; P.25980-007, 2 specimens, 50-54 mm SL, Qatar; P.25988-007, 3 specimens, 55-60 mm SL, Bahrain; P.29813-001, 2 specimens (both c/s), 45-61 mm SL, Persian Gulf; P.31179-001, 3 specimens, 53-67 mm SL, Arabian Gulf, Saudi Arabia; P.31181-001, 14 specimens, $11-28 \mathrm{~mm}$ SL, Arabian Gulf, Saudi Arabia; P.31182-001, 2 specimens, 36-48 mm SL, Arabian Gulf, Saudi Arabia; P.31183-001, 3 specimens, $34-47 \mathrm{~mm} \mathrm{SL}$, Arabian Gülf,-Saudi-Arabia;-P.31184-001, 7 specimens, 18-45 mm SL, Arabian Gulf, Saudi Arabia; P.31185-001, 54 mm SL, Arabian Gulf, Saudi Arabia; P.31186-001, $60 \mathrm{~mm} \mathrm{SL}$, Arabian Gulf, Saudi Arabia; P.31187-001, 74 mm SL, Arabian Gulf, Saudi Arabia.

Paramonacanthus lowei Hutchins, 1997: 30, figures 18, 19.

PARATYPe. P.29775-001 (c/s and skin), $43 \mathrm{~mm} \mathrm{SL}$, Manly Cove, NSW, Australia.

Pervagor marginalis Hutchins, 1986: 23, Plate 2a. PARATYPE. P.28361-001, $50 \mathrm{~mm}$ SL, Marquesas Islands, Pacific Ocean.

Pervagor randalli Hutchins, 1986: 30, Plate 2d, e. Paratrees. P.28357-001, $48 \mathrm{~mm} \mathrm{SL}$, Gulf of Tadjoura, Yemen; P.28358-001 (c/s), 44 mm SL, Musha Island, Yemen.

Pseudomonacanthus galii Waite, 1905: 79, Plate 16. HolotyPE. P.13259-001, 300 mm SL, Shark Bay, WA, Australia.

REMARKS. Although the label with this specimen states that it is "possibly type", there is little doubt it is the holotype. Its length matches that given in the type description, and the specimen closely resembles the illustration of the type. However, there are some discrepancies. The head and body proportions given in the description match neither the proportions of the specimen nor those of the type illustration. A comparison of the proportions with the SL yield the values given and it is likely that this was the body length used rather than TL as stated. The fin ray counts of this specimen match those visible in the type illustration with the exception of the pectoral count (12 vs 10 in the illustration). A further 15 specimens were examined (Hutchins and Smith 1991) and all had 12 pectoral rays. The low pectoral ray count in the type illustration was probably an error on the part of the artist.

SYNONYMY. This species is now known as Meuschenia galii.

Rudarius excelsus Hutchins, 1977: 49, figure 13. Paratype. P.25603-001, 15 mm SL (c/s), Lindeman Island, QId., Australia 


\section{Tetraodontidae}

Canthigaster leoparda Lubbock and Allen, 1979: 88, figures $1,2$.

Paratypes. P.25239-010, $56 \mathrm{~mm}$ SL, Ambon Island, Indonesia; P.26116-003, $51 \mathrm{~mm} \mathrm{SL}$, Christmas Island, Indian Ocean.

Canthigaster ocellicincta Allen and Randall, 1977: 485 , figure $3 b$.

Paratype. P.25283-004, 28 mm SL, Suva, Fiji.

Canthigaster pygmaea Allen and Randall, 1977: 498, figure 15.

PARATYPES. P.25124-001, 36 mm SL, Eilat, Israel; P.25125-001, 2 specimens, 17-31 mm SL, Gulf of Aqaba, Red Sea.

Canthigaster smithae Allen and Randall, 1977: 489, figure $5 c$.

Paratype. P.25126-001, $50 \mathrm{~mm}$ SL, Flic en Flac, Mauritius (missing, presumed lost).

Canthigaster tyleri Allen and Randall, 1977: 497, figure $6 \mathrm{c}$.

PARATYPE. P.25169-001, 58 mm SL, Ambon Island, Indonesia.

Contusus brevicaudus Hardy, 1981: 16, figure 3.

ParatyPes. P.21821-001, $147 \mathrm{~mm}$ SL, Wilson Inlet, WA, Australia; P.22203-001, 182 mm SL, Hardy Inlet, WA, Australia; P.25064-008, 75 mm SL, Hardy Inlet, WA, Australia; P.25183-001, 143 mm SL, Stokes Inlet, WA, Australia; P.25722-001, $185 \mathrm{~mm}$ SL, Mandurah, WA, Australia.

Omegophora cyanopunctata Hardy and Hutchins, 1981: 195, figure 2.

Holotype. P.26942-001, $123 \mathrm{~mm}$ SL, Cape Naturaliste, WA, Australia.

Paratypes. P.5648-001, $121 \mathrm{~mm} \mathrm{SL}$, Middleton Beach, WA, Australia; P.24540-001, $87 \mathrm{~mm} \mathrm{SL}$, Carnac Island, WA, Australia; P.25763-001, 85 $\mathrm{mm}$ SL, Recherche Archipelago, WA, Australia; P.25769-001, 112 mm SL, Recherche Archipelago, WA, Australia; P.26003-004, 2 specimens, 79-86 mm SL, Recherche Archipelago, WA, Australia; P.26564-001, $133 \mathrm{~mm} \mathrm{SL}$, Cape Leeuwin, WA, Australia; P.26928-001, 120 mm SL, Busselton, WA, Australia.

Torquigener pallimaculatus Hardy, 1983b: 20, figure 8.

Paratypes. P.13490-001, $92 \mathrm{~mm} \mathrm{SL}$, Exmouth Gulf, WA, Australia; P.13491-001, $133 \mathrm{~mm} \mathrm{SL}$, Exmouth Gulf, WA, Australia; P.22070-001, 123 mm SL, Rottnest Island, WA, Australia; P.23439001 (skel.), $122 \mathrm{~mm} \mathrm{SL}$, Exmouth Gulf, WA, Australia; P.23440-001, 129 mm SL, Exmouth Gulf, WA, Australia; P.23799-001, 132 mm SL, Exmouth Gulf, WA, Australia; P.25095-026, 2 specimens, 99-118 mm SL, Exmouth Gulf, WA, Australia; P.26929-001, 2 specimens, 103-105 mm SL, Rottnest Island, WA, Australia; P.26293002, $117 \mathrm{~mm} \mathrm{SL}$, eastern Indian Ocean, WA, Australia.

Torquigener paxtoni Hardy, 1983b: 17, figure 7. HoLOTYPE. P.23298-001, 107 mm SL, Shark Bay, WA, Australia.

Paratypes. P.13488-001, $91 \mathrm{~mm}$ SL, Shark Bay, WA, Australia; P.13489-001, 104 mm SL, Shark Bay, WA, Australia; P.23296-001, $59 \mathrm{~mm}$ SL, Shark Bay, WA, Australia; P.23297-001, $82 \mathrm{~mm}$ SL, Shark Bay, WA, Australia; P.23299-001, 110 mm SL, Shark Bay, WA, Australia; P.23300-001, 95 mm SL, Shark Bay, WA, Australia; P.23301001, $90 \mathrm{~mm}$ SL, Shark Bay, WA, Australia; P.23302-001, $75 \mathrm{~mm} \mathrm{SL,} \mathrm{Shark} \mathrm{Bay,} \mathrm{WA,}$ Australia; P.23607-001, 84 mm SL, Carnarvon, WA, Australia; P.23608-001, $72 \mathrm{~mm} \mathrm{SL}$, Carnarvon, WA, Australia; P.23609-001, $76 \mathrm{~mm}$ SL, Carnarvon, WA, Australia; P.23610-001, 78 mm SL, Carnarvon, WA, Australia; P.26771-001 (skel.), $110 \mathrm{~mm}$ SL, Shark Bay, WA, Australia; P.26924-001, 9 specimens, 66-81 mm SL, Houtman Abrolhos, WA, Australia; P.26924-002, $88 \mathrm{~mm}$ SL, Houtman Abrolhos, WA, Australia; P.26925-001, 95 mm SL, Shark Bay, WA, Australia; P.26926-001, $90 \mathrm{~mm} \mathrm{SL}$, Rottnest Island, WA, Australia; P.26927-001, 94 mm SL, Shark Bay, WA, Australia.

Torquigener piosae Whitley, 1955: 56, figure 8. Paratypes. P.3681-001 to P.3686-001, Leighton, WA, Australia.

SYNONYMY. This species is now known as Polyspina piosae.

\section{ACKNOWLEDGEMENTS}

The preparation of this catalogue was facilitated by the earlier efforts of staff in the WAM fish section, N. Sarti and N. Haig. In addition, the following helped with information on material in their care: G.R. Allen (WAM); B. Brown (ANMH); A. Graham (CSIRO); J. Johnson (QM); D.F. Hoese, M. McGrouther and J.R. Paxton (AMS); R.J. McKay (QM); A. Suzumoto (BPBM); J. Thompson (University of Queensland). Help with the location of some of the early WAM catalogues was given by L.A. Smith (WAM). Help with obtaining type descriptions and old correspondence was provided by M. Triffitt and W. Crawford of the WAM Library.

\section{REFERENCES}

Akihito, K. Meguro and Sakamoto, K. (2003). A new species of gobiid fish, Cristatogobius rubripectoralis, 
from Australia. Ichthyological Research 50(2): 117122.

Alexander, W. B. (1917). Description of a new species of fish of the genus Evoxymetopon, Poey. Journal of the Proceedings of the Royal Society of Western Australia 2: 104-105.

Allen, G. R. (1972). The Anemonefishes. Their classification and biology. T. F. H. Publications, Inc. $288 \mathrm{pp}$.

Allen, G. R. (1976a). Descriptions of three new fishes from-Western Australia. Journal of the Royal Society of Western Australia 59(1): 24-30.

Allen, G. R. (1976b). Two new species of damselfishes (Pomacentridae) from Western Australia. Records of the Western Australian Museum 4(2): 133-144.

Allen, G. R. (1977a). A new species of scorpaenid fish (Scorpaenidae) from Western Australia. Proceedings of the Linnaean Society of New South Wales 101(3): 145-148.

Allen, G. R. (1977b). A revision of the plesiopid fish genus Trachinops, with the description of a new species from Western Australia. Records of the Western Australian Museum 5(1): 59-72.

Allen, G. R. (1978). The rainbowfishes of northwestern Australia (family Melanotaeniidae). Tropical Fish Hobbyist 26(10): 91-102.

Allen, G. R. (1979). Chilatherina axelrodi, a new species of rainbowfish (Melanotaeniidae) from Papua New Guinea. Tropical Fish Hobbyist 28(5): 48-49, 52-55.

Allen, G. R. (1980). Two new species of freshwater rainbowfishes (Melanotaeniidae) from Papua New Guinea. Revue Française $d^{\prime}$ Aquariologie 7(2): 43-50.

Allen, G. R. (1981a). A new species of Glossolepis (Pisces: Melanotaeniidae) from fresh waters of Papua New Guinea. Records of the Western Australian Museum 9(3): 301-306.

Allen, G. R. (1981b). Central highland rainbows from Papua New Guinea, with descriptions of two new species (Melanotaeniidae). Tropical Fish Hobbyist 29(5): 20-33, 68-81.

Allen, G. R. (1981c). The "maccullochi species group" of rainbowfishes (Melanotaeniidae) with the description of Melanotaenia papuae, new species. Revue Française d' Aquariologie 8(2): 47-56.

Allen, G. R. (1981d). Popondetta connieae, a new species of rainbowfish (Melanotaeniidae) from Papua New Guinea. Revue Française d' Aquariologie 8(2): 43-46.

Allen, G. R. (1982a). Melanotaenia corona, a new species of rainbowfish from northern New Guinea (Pisces, Atheriniformes, Melanotaeniidae). Bulletin Zoologisch Museum Universiteit van Amsterdam 8(21): 173-176.

Allen, G. R. (1982b). A new species of freshwater rainbowfish (Melanotaeniidae) from Misool Island, Indonesia. Records of the Western Australian Museum 10(2): 105-109.

Allen, G. R. (1982c). Parambassis altipinnis, a new species of freshwater glassfish from western New Guinea (Pisces, Ambassidae). Bulletin of the Zoological Museum of the University of Amsterdam 8(20): 165169.
Allen, G. R. (1982d). Inland fishes of Western Australia. Western Australian Museum, Perth. 86 pp.

Allen, G. R. (1983a). Kiunga ballochi, a new genus and species of rainbowfish (Melanotaeniidae) from Papua New Guinea. Tropical Fish Hobbyist 32(2): 72-77.

Allen, G. R. (1983b). Melanotaenia maylandi, a new species of rainbowfish (Melanotaeniidae) from $\mathrm{New}$ Guinea. Revue Française $d^{\prime}$ Aquariologie 10(3): 8386.

Allen, G. R. (1983c). A new genus and species of wrasse (Pisces: Labridae) from Rowley Shoals, Western Australia.Revue Erançaise d' Aquariologie 10(2): 4346.

Allen, G. R. (1984a). A new genus and species of anthiid fish from Papua New Guinea. Revue Française $d^{\prime}$ Aquariologie 11(2): 47-50.

Allen, G. R. (1984b). A new species of freshwater grunter (Pisces: Teraponidae) from New Guinea. Records of the Western Australian Museum 11(4): 393-397.

Allen, G. R. (1985a). Descriptions of two new species of freshwater catfishes (Plotosidae) from Papua New Guinea. Records of the Western Australian Museum 12(3): 247-256.

Allen, G. R. (1985b). Three new rainbowfishes (Melanotaeniidae) from West Papua, Indonesia and Papua New Guinea. Revue Française d' Aquariologie 12(2): 53-62.

Allen, G. R. (1985c). A new species of cardinalfish (Apogonidae) from northern Australia and the Aru Islands. The Beagle (Occasional Papers of the Northern Territory Museum of Arts and Sciences) 2(1): 11-15.

Allen, G. R. (1987a). Melanotaenia iris, a new freshwater rainbowfish (Melanotaeniidae) from Papua New Guinea with notes on the fish fauna in head waters. Japanese Journal of Ichthyology 34(1): 15-20.

Allen, G. R. (1987b). Descriptions of three new pseudochromid fishes of the genus Pseudoplesiops from Australia and surrounding regions. Records of the Western Australian Museum 13(2): 249-261.

Allen, G. R. (1987c). New Australian fishes. Part 4. A new species of Steeneichthys (Plesiopidae). Memoirs of the Museum of Victoria 48(1): 13-14.

Allen, G. R. (1987d). New Australian fishes. Part 2. Four new species of Apogonidae. Memoirs of the Museum of Victoria $48(1)$ : 3-8.

Allen, G. R. (1987e). Chrysiptera sinclairi, a new species of damselfish from the tropical western Pacific Ocean. Revue Française d' Aquariologie 13(4): 107-110.

Allen, G. R. (1987f). A new species of pomacentrid fish with notes on other damselfishes of the Kermadec Islands. Records of the Western Australian Museum 13(2): 263-273.

Allen, G. R. (1987g). New Australian fishes. Part 3. A new species of Pomacentrus (Pomacentridae). Memoirs of the Museum of Victoria 48(1): 9-11.

Allen, G. R. (1990). Les poissons arc-en-ciel (Melanotaeniidae) de la Péninsule de Vogelkop, West Papua, Indonesia, avec description de trois nouvelles espèces. Revue Française d'Aquariologie 16(4): 101112. 
Allen, G. R. (1991a). Damselfishes of the world. Mergus Publishers, Melle, Germany. 271 pp.

Allen, G. R. (1991b). Field guide to the freshwater fishes of New Guinea. Publication No. 9 of the Christensen Research Institute, Madang, Papua New Guinea. 268 pp.

Allen, G. R. (1992a). A new species of cardinalfish (Apogonidae) from northern Australia. Records of the Western Australian Museum 15(4): 697-701.

Allen, G. R. (1992b). A new species of damselfish (genus Pomacentrus) from north-western Australia. Records of the Western Australian Museum 15(4): 691-695.

Allen, G. R. (1993a). Cardinalfishes (Apogonidae) of Madang Province, Papua New Guinea, with descriptions of three new species. Revue Française $d^{\prime}$ Aquariologie 20(1): 9-20.

Allen, G. R. (1993b). Two new species of damselfishes (Pomacentrus), with comments on the validity of two additional pomacentrid fishes. Revue Française $d^{\prime}$ Aquariologie 20(1): 21-26.

Allen, G. R. (1995a). Pseudochromis howsoni, a new species of dottyback fish (Pseudochromidae) from Ashmore Reef, Timor Sea. Revue Française d' Aquariologie 21(3-4): 83-85.

Allen, G. R. (1995b). A new species of cardinalfish (Apogon: Apogonidae) from northwestern Australia. Records of the Western Australian Museum 17: 177180.

Allen, G. R. (1995c). Two new species of damselfishes (Pomacentridae) from Indonesian seas. Revue Française d'A Aquariologie 21(3-4): 86-90.

Allen, G. R. (1995d). A new species of wrasse (Labridae: Cirrhilabrus) from Western Australia. Revue Française d'A Aquariologie 22(1-2): 14-18.

Allen, G. R. (1995e). Thalassoma robertsoni, a new species of wrasse (Labridae) from Clipperton Island tropical eastern Pacific Ocean. Revue Française $d^{\prime}$ Aquariologie 22(3-4): 75-79.

Allen, G. R. (1996a). Two new species of rainbowfishes (Melanotaenia: Melanotaeniidae), from the Kikori River system, Papua New Guinea. Revue Française $d^{\prime}$ Aquariologie 23(1-2): 9-16.

Allen, G. R. (1996b). Oxyeleotris caeca, a new species of blind cave fish (Eleotridae) from Papua New Guinea. Revue Française $d^{\prime}$ Aquariologie 23(1-2): 43-46.

Allen, G. R. (1997a). A new species of rainbowfish (Melanotaenia: Melanotaeniidae), from the Lakekamu Basin, Papua New Guinea. Revue Française d' Aquariologie 24(1-2): 37-42.

Allen, G. R. (1997b). Lentipes watsoni, a new species of freshwater goby (Gobiidae) from Papua New Guinea. Ichthyological Exploration of Freshwaters 8(1): 33-40.

Allen, G. R. (1998a). A review of the marine catfish genus Paraplotosus (Plotosidae) with the description of a new species from north-western Australia. Raffles Bulletin of Zoology 46(1): 123-124.

Allen, G. R. (1998b). A new genus and species of rainbowfish (Melanotaeniidae) from fresh waters of West Papua, Indonesia, Indonesia. Revue Française $d^{\prime}$ Aquariologie 25(1-2): 11-16.

Allen, G. R. (1998c). A new species of cardinalfish
(Apogonidae) from the Komodo Islands, Indonesia. Revue Française $d^{\prime}$ Aquariologie 25(1-2): 27-30.

Allen, G. R. (1999a). Altrichthys, a new genus of damselfish (Pomacentridae) from Philippine Seas with description of a new species. Revue Française $d^{\prime}$ Aquariologie 26(1-2): 23-28.

Allen, G. R. (1999b). Three new species of damselfishes (Pomacentridae) from Indonesia and eastern Papua New Guinea. Revue Française d'Aquariologie 25(34): 99-106.

Allen, G. R. (1999c). Descriptions of a new wrasse (Pisces: Labridae; Cirrhilabrus) from north-western Australia. Revue Française d' Aquariologie 25(3-4): 119-122.

Allen, G. R. (1999d). Myersina larsonae, a new species of goby (Pisces: gobiidae) from north-western Australia. Revue Française d' Aquariologie 26(1-2): 49-51.

Allen, G. R. (2000). Description of a new wrasse (Pisces: Labridae: Cirrhilabrus) from northern Sumatra, Indonesia. Aqua, Journal of Ichthyology and Aquatic Biology 4(2): 45-50.

Allen, G. R. (2001a). A new species of rainbowfish (Glossolepis: Melanotaeniidae) from West Papua, Indonesia, Indonesia. Journal of the Australia and New Guinea Fishes Association 15(3): 766-775.

Allen, G. R. (2001b). Two new species of cardinalfishes (Apogonidae) from the Raja Ampat Islands, Indonesia. Aqua, Journal of Ichthyology and Aquatic Biology 4(4): 143-149.

Allen, G. R. (2001c). Description of two new gobies (Eviota, Gobiidae) from Indonesian seas. Aqua, Journal of Ichthyology and Aquatic Biology 4(4): 125130.

Allen, G. R. (2002). Descriptions of two new species of damselfishes (Pomacentridae: Pomacentrus) from Madagascar. Aqua, Journal of Ichthyology and Aquatic Biology 6(2): 45-52.

Allen, G. R. (2003). Allomogurnda, a new genus of gudgeon (Eleotridae) from fresh waters of New Guinea, with descriptions of seven new species. Fishes of Sahul $17(3,4)$ : 978-997.

Allen, G. R. (2004a). Kiunga bleheri, a new blue-eye (Pisces: Pseudomugilidae) from fresh waters of Papua New Guinea. Aqua, Journal of Ichthyology and Aquatic Biology 8(2): 79-85.

Allen, G. R. (2004b). Toxotes kimberleyensis, a new species of archerfish (Pisces: Toxotidae) from fresh waters of Western Australia. Records of the Australian Museum 56(2): 225-230.

Allen, G. R. (2004c). Pomacentrus aurifrons, a new species of damselfish (Pomacentridae) from Melanesia. Zootaxa 399: 1-7.

Allen, G. R. (2004d). Two new species of gobiid fishes (Lentipes and Stenogobius) from fresh waters of Milne Bay Province, Papua New Guinea. Fishes of Sahul 18 (4): 87-96.

Allen, G. R. and Adrim, M. (1992). A new species of damselfish (Chrisiptera: Pomacentridae) from West Papua, Indonesia, Indonesia. Records of the Western Australian Museum 16(1): 103-108.

Allen, G. R. and Adrim, M. (2000). Amblypomacentrus clarus, a new species of damselfish (Pomacentridae) from the Banggai Islands, Indonesia. Records of the 
Western Australian Museum 20(1): 51-55.

Allen, G. R. and Ayling, A. M. (1987). New Australian fishes. Part 5. A new species of Acanthurus (Acanthuridae). Memoirs of the Museum of Victoria 48(1): 15-16.

Allen, G. R. and Bailey, S. (2002). Chrysiptera albata, a new species of damselfish (Pomacentridae) from the Phoenix Islands, Central Pacific Ocean. Aqua, Journal of Ichthyology and Aquatic Biology 6(1): 39-43.

Allen, G. R. and Boeseman, M. (1982). A collection of freshwater fishes from western New Guinea with descriptions of two new species (Gobiidae-and Eleotridae). Records of the Western Australian Museum 10(2): 67-103.

Allen, G. R. and Burhanuddin (1976). Anthias hutomoi, a new species of serranid fish from Indonesia (Perciformes, Serranidae). Marine Research in Indonesia 16: 45-50.

Allen, G. R. and Coates, D. (1990). A new species of freshwater eleotridid fish from northern Papua New Guinea. Records of the Western Australian Museum Suppl. 34: 131-137.

Allen, G. R. and Cross, N. J. (1980). Descriptions of five new rainbowfishes (Melanotaeniidae) from New Guinea. Records of the Western Australian Museum 8(3): 377-396.

Allen, G. R. and Cross, N. J. (1982). Rainbowfishes of Australia and Papua New Guinea. T. F. H. Publications Inc., New Jersey. 141 pp.

Allen, G. R. and Cross, N. J. (1983). A new species and two new records of squirrelfishes (Holocentridae) from the eastern Indian Ocean and Australia. Revue Française d' Aquariologie 10(1): 5-8.

Allen, G. R. and Emery, A. R. (1985). A review of the pomacentrid fishes of the genus Stegastes from the Indo-Pacific, with descriptions of two new species. Indo-Pacific Fishes 3: 1-31.

Allen, G. R. and Erdmann, M. V. (2005). Chromis xouthos, a new species of damselfish (Pomacentridae) from the East Andaman Sea and central Indian Ocean. Aqua, Journal of Ichthyology and Aquatic Biology 10 (3): 89-94.

Allen, G. R. and Erdmann, M. (2006a). Cirrhilabrus cenderawasih, a new wrasse (Pisces: Labridae) from Papua, Indonesia. Aqua, Journal of Ichthyology and Aquatic Biology 11 (3), 89-95.

Allen, G. R. and Erdmann, M. (2006b). Paracheilinus walton, a new flasherwrasse (Perciformes: Labridae) from Papua, Indonesia with a key to the species of Paracheilinus. Aqua, Journal of Ichthyology and Aquatic Biology 12 (1), 11-18.

Allen, G. R. and Erdmann, M. V. (2006c). Pterocaesio flavifasciata, a new species of fusilier (Teleostei: Caesionidae) from Sumatra, Indonesia. Aqua, Journal of Ichthyology and Aquatic Biology 12 (1), 27-30.

Allen, G. R. and Feinberg, M. N. (1998). Descriptions of a new genus and four new species of freshwater catfishes (Plotosidae) from Australia. Aqua, Journal of Ichthyology and Aquatic Biology 3(1): 9-18.

Allen, G. R. and Heemstra, P. C. (1976). Cheilodactylus rubrolabiatus, a new species of morwong (Pisces: Cheilodactylidae) from Western Australia, with a key to cheilodactylid fishes of Australia. Records of the Western Australian Museum 4(4): 311-325.

Allen, G. R. and Hoese, D. F. (1975). A review of the pomacentrid fish genus Parma, with descriptions of two new species. Records of the Western Australian Museum 3(4): 261-293.

Allen, G. R. and Hoese, D. F. (1986). The eleotrid fishes of Lake Kutubu, Papua New Guinea with descriptions of four new species. Records of the Western Australian Museum 13(1): 79-100.

Allen, G. R. and Hoese, D. F. (1991). A review of the -genus Mogunnda (Pisces: Eleotrididae) from New Guinea with descriptions of three new species. Ichthyological Exploration of Freshwaters 2(1): 31-46.

Allen, G. R., Hortle, K G. and Renyaan, S. J. (2000). Freshwater Fishes of the Timika Region, New Guinea. PT Freeport Indonesia. 175 pp.

Allen, G. R. and Ivantsoff, W. (1986). Deux nouvelles espèces de blue-eyes (Pseudomugil: Melanotaeniidae) de Nouvelle-Guinée. Revue Française d' Aquariologie 12(3): 85-88.

Allen, G. R., Ivantsoff, W., Shepherd, M. A. and Renyaan, S. J. (1998). Pseudomugil pellucidus (Pisces: Pseudomugilidae), a newly discovered blue-eye from Timika-Tembaqapura region, West Papua, Indonesia. Aqua, Journal of Ichthyology and Aquatic Biology 3(1): 1-8.

Allen, G. R. and Jebb, M. (1993). A collection of fishes from the Upper Purari River system, Papua New Guinea, with descriptions of two new species (Terapontidae and Eleotrididae). Ichthyological Exploration of Freshwaters 4(3): 233-240.

Allen, G. R. and Jenkins, A. P. (1999a). Two new species of Mogurnda (Osteichthyes: Eleotrididae) from the Etna Bay Region, West Papua, Indonesia, Indonesia. Ichthyological Exploration of Freshwaters 10(3): 237 246.

Allen, G. R. and Jenkins, A. P. (1999b). A review of the Australian freshwater gudgeons, genus Mogurnda (Eleotridae) with descriptions of three new species. Aqua, Journal of Ichthyology and Aquatic Biology 3(4): 141-156.

Allen, G. R., Jenkins, A. P. and Renyaan, S. J. (1999). Mogurnda wapoga, a new species of freshwater gudgeon (Eleotridae) from West Papua, Indonesia. Revue Française d' A quariologie 26(1-2): 63-66.

Allen, G. R. and Kailola, P. J. (1979). Glossolepis wanamensis, a new species of freshwater rainbowfish (Melanotaeniidae) from New Guinea. Revue Française d' Aquariologie 6(2): 39-44.

Allen, G. R. and Kuiter, R. H. (1976). A review of the plesiopid fish genus Assessor, with descriptions of two new species. Records of the Western Australian Museum 4(3): 201-215.

Allen, G. R. and Kuiter, R. H. (1994). Descriptions of two news [sic] species of cardinalfishes (Apogonidae) from Malaysia and Indonesia. Revue Française $d^{\prime}$ Aquariologie 21(1-2): 19-23.

Allen, G. R. and Kuiter, R. H. (1999). Descriptions of two new wrasses of the genus Cirrhilabrus (Labridae) from Indonesia. Aqua, Journal of Ichthyology and Aquatic Biology 3(4): 133-140. 
Allen, G. R. and Kuiter, R. H. (2004). Dunckerocampus naia, a new species of pipefish (Pisces: Syngnathidae) from the western Pacific. Aqua, Journal of Ichthyology and Aquatic Biology 9(1): 1-6.

Allen, G. R., Kuiter, R. H. and Randall, J. E. (1994). Descriptions of five new species of cardinalfishes (Apogonidae: Apogon) from Maumere Bay, Flores, Indonesia and surrounding regions. Revue Française $d^{\prime}$ Aquariologie 21(1-2): 27-38.

Allen, G. R. and Larson, H. K. (1979). Parma bicolor, a new species of damselfish from southwestern Australia. Revue Française d' Aquariologie 6(1): 1114.

Allen, G. R., Larson, H. K. and Midgley, S. H. (1993). A new species of Scortum Whitley (Pisces: Terapontidae) from the Northern Territory, Australia. The Beagle (Occasional Papers of the Northern Territory Museum of Arts and Sciences) 10(1): 71-74.

Allen, G. R. and Lubbock, R. (1976). Glyphidodontops springeri, a new species of damselfish (Pomacentridae) from the Philippine and Molucca Islands. Proceedings of the Biological Society of Washington 88(31): 345-350.

Allen, G. R. and Merrick, J. R. (1984). A new species of freshwater grunter (Pisces: Teraponidae) from Northern Australia. The Beagle (Occasional Papers of the Northern Territory Museum of Arts and Sciences) 1(8): 75-80.

Allen, G. R. and Moore, R. (1981). Pseudomugil paludicola, a new species of freshwater blue-eye (Melanotaeniidae) from Papua New Guinea. Revue Française d' A quariologie 7(4): 105-108.

Allen, G. R. and Morrison, S. M. (1996). A new species of cardinalfish (Apogonidae) from northern Australia. Records of the Western Australian Museum 17: 439442.

Allen, G. R. and Moyer, J. T. (1980). Ellerkeldia wilsoni, a new species of serranid fish from southwestern Australia. Japanese Journal of Ichthyology 26(4): 329333.

Allen, G. R. and Munday, P. (1995). Description of four new gobies (Gobiidae) from the western Pacific Ocean. Revue Française $d^{\prime}$ Aquariologie 22(3-4): 99104.

Allen, G. R. and Rajasuriya, A. (1995). Chrysiptera kuiteri, a new species of damselfish (Pomacentridae) from Indonesia and Sri Lanka. Records of the Western Australian Museum 17(3): 283-286.

Allen, G. R. and Randall, J. E. (1977). Review of the sharpnose pufferfishes (subfamily Canthigasterinae) of the Indo-Pacific. Records of the Australian Museum 30(17): 475-517.

Allen, G. R. and Randall, J. E. (1980). A review of the damselfishes (Teleostei: Pomacentridae) of the Red Sea. Israel Journal of Zoology 29(1): 1-98.

Allen, G. R. and Randall, J. E. (1985a). A new genus and species of plesiopid fish from Western Australia and the central-south Pacific Ocean. Records of the Western Australian Museum 12(2): 185-191.

Allen, G. R. and Randall, J. E. (1985b). A new species of damselfish (Pomacentridae) from eastern Australia and the Norfolk Island Ridge. Records of the Western Australian Museum 12(2): 241-245.
Allen, G. R. and Randall, J. E. (1990). Hypoplectrodes cardinalis, a new name for the serranid fish $H$. ruber (Allen) from southwestern Australia, with discussion of $H$. semicinctus from Juan Fernandez Islands. Revue Française d' Aquariologie 17(2): 45-46.

Allen, G. R. and Randall, J. E. (1993). Three new species of cardinalfishes (Apogonidae) from Australia and adjacent seas. Revue Française d' Aquariologie 19(4): 107-114.

Allen, G. R. and Randall, J. E. (1994). A new species of cardinalfish (Apogon: Apogonidae) from Arabian Seas. Revue Française d' Aquariologie 21(1-2): 24-26.

Allen, G. R. and Randall, J. E. (1996). Three new species of wrasses (Labridae: Cirrhilabrus) from Papua New Guinea and the Solomon Islands. Revue Française $d^{\prime}$ Aquariologie 23(3-4): 101-112.

Allen, G. R. and Randall, J. E. (2002a). Four new IndoPacific species of cardinalfishes (Apogonidae). Aqua, Journal of Ichthyology and Aquatic Biology 5(3): 115126.

Allen, G. R. and Randall, J. E. (2002b). A review of the leucogaster species complex of the Indo-Pacific pomacentrid genus Amblyglyphidodon, with descriptions of two new species. Aqua, Journal of Ichthyology and Aquatic Biology 5(4): 139-152.

Allen, G. R. and Randall, J. E. (2002c). A new species of wrasse (Labridae: Choerodon) from the tropical western Pacific. Aqua, Journal of Ichthyology and Aquatic Biology 5(3): 109-113.

Allen, G. R. and Randall, J. E. (2004). Two new species of damselfishes (Pomacentridae: Chromis) from Indonesian seas. Aqua, Journal of Ichthyology and Aquatic Biology 9(1): 17-24.

Allen, G. R. and Randall, J. E. (2005a). A new species of damselfish (Pomacentrus: Pomacentridae) from Fiji. Aqua, Journal of Ichthyology and Aquatic Biology 10 (3): 95-102.

Allen, G. R. and Randall, J. E. (2005b). Exyrias akihito, A new species of coral-reef goby (Gobiidae) from the western Pacific. The Raffles Bulletin of Zoology 53 (2): 75-79

Allen, G. R. and Randall, J. E. (2006). Vanderhorstia nobilis, a new species of shrimpgoby from Indonesia and the Philippines. Aqua, Journal of Ichthyology and Aquatic Biology 12 (1), 39-44.

Allen, G. R., Randall, J. E. and Carlson, B. A. (2003). Cirrhilabrus marjorie, a new wrasse (Pisces: Labridae) from Fiji. Aqua, Journal of Ichthyology and Aquatic Biology 7(3): 113-118.

Allen, G. R. and Renyaan, S. J. (1996a). Chilatherina pricei, a new species of rainbowfish (Melanotaeniidae) from West Papua, Indonesia. Revue Française d' Aquariologie 23(1-2): 5-8.

Allen, G. R. and Renyaan, S. J. (1996b). Three new species of rainbowfishes (Melanotaeniidae) from the Triton Lakes, West Papua, Indonesia, New Guinea. Aqua, Journal of Ichthyology and Aquatic Biology 2(2): 13-24.

Allen, G. R. and Renyaan, S. J. (1996c). Eleotrid fishes of the Triton Lakes, West Papua, Indonesia, with description of four new species. Revue Française $d^{\prime}$ Aquariologie 23(1-2): 47-56. 
Allen, G. R. and Renyaan, S. J. (1998). Three new species of rainbowfishes (Melanotaeniidae) from West Papua, Indonesia, Indonesia. Aqua, Journal of Ichthyology and Aquatic Biology 3(2): 69-80.

Allen, G. R. and Renyaan, S. J. (1999). Pseudomugil ivantsoffi (Pseudomugilidae), a new species of blueeye from the Timika region, West Papua, Indonesia. Aqua, Journal of Ichthyology and Aquatic Biology 3(4): 163-168.

Allen, G. R. and Robertson, D. R. (1991a). Quatre espèces nouvelles d'Opisthognathidae (jawfishes) $d u$ Pacifique oriental tropical-Revtue-Française $d^{\prime}$ Aquariologie 18(2): 47-52.

Allen, G. R. and Robertson, D. R. (1991b). Description of two new genera and four new species of triplefins (Pisces: Tripterygiidae) from the tropical eastern Pacific. Revue Française d' Aquariologie 18(3): 79-82.

Allen, G. R. and Robertson, D. R. (1992a). Serranus socorroensis, a new species of serranid fish from the tropical eastern Pacific Ocean. Revue Française d' Aquariologie 19(1-2): 37-40.

Allen, G. R. and Robertson, D. R. (1992b). Deux nouvelles espèces de Girelles (Labridae: Halichoeres) du Pacifique oriental tropical. Revue Française $d^{\prime}$ Aquariologie 19(1-2): 47-52.

Allen, G. R. and Robertson, D. R. (1992c). Three new species of triplefins (Pisces: Tripterygiidae) from Malpelo and Socorro Islands, in the tropical eastern Pacific. Revue Française d' Aquariologie 19(1-2): 5356.

Allen, G. R. and Robertson, D. R. (1995a). Pseudogramma axelrodi, a new species of serranid fish from the tropical eastern Pacific Ocean. Tropical Fish Hobbyist 44(1): 72-75.

Allen, G. R. and Robertson, D. R. (1995b). Xyrichthys wellingtoni, a new species of wrasse (Labridae) from Clipperton Island, tropical eastern Pacific Ocean. Revue Française d' Aquariologie 22(3-4): 80-82.

Allen, G. R. and Sarti, N. (1983). Pseudomugil cyanodorsalis, une nouvelle espèce de blue-eye (Melanotaeniidae) d'Australie nord-occidentale. Revue Française d'A Auariologie 10(2): 47-50.

Allen, G. R. and Smith, K. N. (1992). A new species of damselfish (Pomacentridae: Stegastes) from Ascension Island, Atlantic Ocean. Records of the Western Australian Museum 16(1): 113-117.

Allen, G. R. and Starck, W. A. II (1975). Cephalopholis xanthopterus, a new species of serranid fish from Indonesia, Melanesia and the Great Barrier Reef. Records of the Western Australian Museum 3(3): 245249.

Allen, G. R. and Starck, W. A. II (1982). The anthiid fishes of the Great Barrier Reef, Australia, with the description of a new species. Revue Française $d^{\prime}$ Aquariologie 9(2): 47-56.

Allen, G. R. and Steene, R. C. (2004). Chaetodontoplus vanderloosi, a new species of angelfish (Pomacanthidae) from Papua New Guinea. Aqua, Journal of Ichthyology and Aquatic Biology 8(1): 2330.

Allen, G. R. and Talbot, F. H. (1985). Review of the snappers of the genus Lutjanus (Pisces: Lutjanidae) from the Indo-Pacific, with the description of a new species. Indo-Pacific Fishes 11: 1-87.

Allen, G. R. and Woods, L. P. (1980). A review of the damselfish genus Stegastes from the eastern Pacific with the description of a new species. Records of the Western Australian Museum 8(2): 171-198.

Allen, G. R. and Wright, J. E. (2003). Description of a new species of damselfish (Pomacentridae: Pomacentrus) from Rodrigues Island, Indian Ocean. Aqua, Journal of Ichthyology and Aquatic Biology 7(4): 133-138.

Amaoka, K. and Arai, M. (1998). Redescription of a rare bothid, Asterorhombus bleekeri (Macleay), and description of a new species of Asterorhombus from northwestern Australia (Teleostei: Pleuronectiformes). Ichthyological Research 45(3): 249-257.

Amaoka, K., Arai, M. and Gomon, M. F. (1997). A new species of Arnoglossus (Pleuronectiformes: Bothidae) from the southwestern coast of Australia. Ichthyological Research 44(2): 131-136.

Anderson, M. E. (2005). Three new species of Microbrotula (Teleostei: Ophidiiformes; Bythitidae) from the Indo-West Pacific. Zootaxa 1006: 33-42.

Anderson, W. D. Jr. and Heemstra, P. C. (1989). Ellerkeldia, a junior synonym of Hypoplectrodes, with redescriptions of the type species of the genera (Pisces: Serranidae: Anthiinae). Proceedings of the Biological Society of Washington 102(4): 1001-1017.

Ayling, A. M. and Paxton, J. R. (1983). Odax cyanoallix, a new species of odacid fish from northern New Zealand. Copeia 1983(1): 95-101.

Ayling, A. M. and Russell, B. C. (1977). The labrid fish genus Pseudojuloides, with description of a new species. Australian Zoologist 19(2): 169-178.

Baldwin, C. C. and Johnson, G. D. (1999). Paxton concilians: a new genus and species of pseudamine apogonid (Teleostei: Percoidei) from northwestern Australia: The sister group of the enigmatic Gymnapogon. Copeia 1999(4): 1050-1071.

Bath, H. (1992). Zwei neue Arten der Gattung Salarias Cuvier 1817 von den Philippinen und von Westaustralien (Pisces: Blenniidae). Senckenbergiana Biologica 72(4): 225-236.

Bath, H. and Hutchins, J. B. (1986). Die Blenniini des australischen Raums und Neuseelands mit Beschreibung einer neuen Art und einer neuen Unterart (Pisces: Blenniidae). Senckenbergiana Biologica 66(4): 167-213.

Bleeker, P. (1856). Carcharias (Prionodon) amblyrhynchos, eene nieuwe haaisoort, gevangen nabij het eiland Solombo. Natuurkundig Tijdschrift voor Nederlandsch Indië 10: 467-468.

Bleeker, P. (1874). Révision des espèces indoarchipélagiques du groupe des Apogonini. Natuurkundige Verhandelingen der Hollandsche Maatschappij der Wetenschappen Harlem (Ser. 3). 2(1): 1-82.

Bloch, M. E. and Schneider, J. G. (1801). M. E. Blochii, Systema Ichthyologiae iconibus cx illustratum. Post obitum auctoris opus inchoatum absolvit, correxit, interpolavit Jo. Gottlob Schneider, Saxo. Berolini. Sumtibus Auctoris Impressum et Bibliopolio Sanderiano Commissum. 1-584 pp. 
Böhlke, J. E. and Randall, J. E. (1981). Four new garden eels (Congridae, Heterocongrinae) from the Pacific and Indian oceans. Bulletin of Marine Science 31(2): 366-382.

Bruner, J. C. and Arnam, S. (1979). Chromis woodsi, a new species of damselfish (Pomacentridae) from the western Indian Ocean with a redescription of Chromis axillaris (Bennett), 1831. Fieldiana Zoology 73(3): 49-63.

Burgess, W. E. (1981). Pomacentrus alleni and Amphiprion thiellei, two new species of pomacentrids (Pisces: Pomacentridae) from the IndoPacific. Tropical Fish Hobbyist 30(3): 68-69, 72-73.

Bussing, W. A. (1991). A new genus and two new species of tripterygiid fishes from Costa Rica. Revista Biologia Tropical 39(1): 77-85.

Cantor, T. E. (1849). Catalogue of Malayan fishes. Journal of the Asiatic Society of Bengal 18(2): 983-1443.

Carpenter, K. E. (1987). Revision of the Indo-Pacific fish family Caesionidae (Lutjanoidea), with descriptions of five new species. Indo-Pacific Fishes 15: 1-56.

Carpenter, K. E. and Randall, J. E. (2003). Lethrinus ravus, a new species of emperor fish (Perciformes: Lethrinidae) from the western Pacific and eastern Indian Oceans. Zootaxa 240: 1-8.

Carvalho, M. R., de and Séret, B. (2002). Narcine lasti, a new species of numbfish from western Australia and Indonesia (Chondrichthyes: Torpediniformes: Narcinidae). Records of the Western Australian Museum 20: 393-408.

Castle, P. H. J. and Randall, J. E. (1999). Revision of IndoPacific garden eels (Congridae: Heterocongrinae), with descriptions of five new species. Indo-Pacific Fishes 30: 1-52.

Castelnau, F. L. (1873). Contribution to the ichthyology of Australia. Nos. III thru IX. Proceedings of the Zoological Acclimitisation Society of Victoria 2: 37158.

Chapleau, F. and Renaud, C. B. (1993). Paraplagusia sinerama (Pleuronectiformes: Cynoglossidae), a new Indo-Pacific tongue sole with a revised key to species of the genus. Copeia 1993(3): 798-807.

Chapleau, F., Renaud, C. B. and Kailola, P. J. (1991). Paraplagusia longirostris, a new flatfish (Cynoglossidae) from Australia and Papua New Guinea. Japanese Journal of Ichthyology 38(3): 239244.

Choat, J. H. and Randall, J. E. (1986). A review of the parrotfishes (family Scaridae) of the Great Barrier Reef of Australia with description of a new species. Records of the Australian Museum 38(4): 175-228.

Cohen, D. M. and Hutchins, J. B. (1982). Description of a new Dinematichthys (Ophidiiformes: Bythitidae) from Rottnest Island, western Australia. Records of the Western Australian Museum 9(4): 341-347.

Cohen, D. M. and Nielsen, J. G. (2002). Diplacanthopoma kreffti (Pisces, Bythitidae), a new species from the northwest Australian shelf, with comments on the name $D$. alcockii Goode and Bean, 1896. Archive of Fishery and Marine Research 50(1): 11-15.

Cohen, D. M. and Robins, C. R. (1986). A review of the ophidiid fish genus Sirembo with a new species from
Australia. Memoirs of the Queensland Museum 22(2): 253-263.

Collette, B. B. (1985). Zenarchopterus ornithocephala, a new species of freshwater halfbeak (Pisces: Hemiramphidae) from the Vogelkop Peninsula of New Guinea. Proceedings of the Biological Society of Washington 98(1): 107-111.

Collette, B. B. and Russo, J. L. (1981). A revision of the scaly toadfishes, genus Batrachoides, with descriptions of two new species from the eastern Pacific. Bulletin of Marine Science 31(2): 197-233.

Compagno, L. J. V. and Stevens, J. D. (1993). Atelomycterus fasciatus n. sp., a new catshark (Chondrichthyes: Carcharhiniformes: Scyliorhinidae) from tropical Australia. Records of the Australian Museum 45(2): 147-169.

Compagno, L. J. V. and Stevens, J. D. (1993). Hemitriakis falcata n. sp. and $H$. abdita n. sp., two new houndsharks (Carcharhiniformes: Triakidae) from Australia. Records of the Australian Museum 45(2): 195-220.

Compagno, L. J. V., Stevens, J. D. and Last, P. R. (1999). Australian spotted catsharks of the genus Asymbolus (Carcharhiniformes: Scyliorhinidae). Part 1: Descriptions of three new species from Western Australia, pp. 2-18. In: Last, P. R. (ed.) Australian catsharks of the genus Asymbolus (Carcharhiniformes: Scyliorhinidae). CSIRO Maine Laboratory Report 239: 1-35.

Cressey, R. F. (1981). Revision of Indo-West Pacific lizardfishes of the genus Synodus (Pisces: Synodontidae). Smithsonian Contributions to Zoology 342: 1-53.

Crowley, L. E. L. M. and Ivantsoff, W. (1988). A new species of Australian Craterocephalus (Pisces: Atherinidae) and redescription of four other species. Records of the Western Australian Museum 14(2): 151-169.

Crowley, L. E. L. M. and Ivantsoff, W. (1990a). A review of species previously identified as Craterocephalus eyresii (Pisces: Atherinidae). Proceedings of the Linnaean Society of New South Wales 112(2): 87-103.

Crowley, L. E. L. M. and Ivantsoff, W. (1990b). A second hardyhead, Craterocephalus gloveri (Pisces: Atherinidae), from Dalhousie Springs, central Australia. Ichthyological Exploration of Freshwaters 1(2): 113-122.

Crowley, L. E. L. M., Ivantsoff, W. and Allen, G. R. (1991). Freshwater fishes of the genus Craterocephalus (Atherinidae) from the southern drainages of Papua New Guinea and West Papua, Indonesia with reference to $C$. s. stercusmuscarum from Australia. Records of the Western Australian Museum 15(1): 33-52.

Crowley, L. E. L. M., Ivantsoff, W. and Allen, G. R. (1995). Description of a new species of hardyhead, Craterocephalus fistularis, (Pisces: Atherinidae) from West Papua, Indonesia. Records of the Western Australian Museum 17(3): 325-329.

Cuvier, G. (1816). Le Règne Animal distribué d'après son organisation pour servir de base à l'histoire naturelle des animaux et d'introduction à l'anatomie comparée. Les reptiles, les poissons, les mollusques et les annélides. Edition 1. 532 pp. 
Dawson, C. E. (1978a). Description of a new western Australian pipefish (Choeroichthys latispinosus), with notes on Syngnathus tuckeri Scott and Nannocampichthys Hora and Mukerji. Records of the Western Australian Museum 6(4): 413-421.

Dawson, C. E. (1978b). Syngnathus parvicarinatus, a new Australian pipefish, with notes on $S$. sauvagei (Whitley) and Leptonotus caretta (Klunzinger). Copeia 1978(2): 288-293.

Dawson, C. E. and Allen, G. R. (1978). Synopsis of the 'finless' pipefish genera (Penetopteryx, Apterygocampus and-Enchelyocampus, gen. nov.). Records of the Western Australian Museum 6(4): 391411.

Dawson, C. E. and Allen, G. R. (1981). Micrognathus Spinirostris, a new Indo-Pacific pipefish (Syngnathidae). Journal of the Royal Society of Western Australia 64(2): 65-68.

de Buen, F. (1959). Lampreas, tiburones, rayas y peces en la Estacion de Biologia Marina de Montemar, Chile. (Primera contribucion). Revista de Biología Marina Valparaiso 9(1-3): 3-200.

Dooley, J. K. and Kailola, P. J. (1988). Four new tilefishes from the northeastern Indian Ocean, with a review of the genus Branchiostegus. Japanese Journal of Ichthyology 35(3): 247-260.

Dor, M. and Allen, G. R. (1977). Neopomacentrus miryae, a new species of pomacentrid fish from the Red Sea. Proceedings of the Biological Society of Washington 90(1): 183-188.

Eschmeyer, W. N. and Allen, G. R. (1978). Neoaploactis tridorsalis, a new genus and species of fish from the Great Barrier Reef, Australia (Scorpaeniformes: Aploactinidae). Records of the Western Australian Museum 6(4): 443-448.

Eschmeyer, W. N., Ferraris, C. J. Jr, Dang Hoang, M., and Long, D. J. (2007). Catalog of Fishes. Part 1: Species of Fishes. http://www.calacademy.org/research/ ichthyology/catalog/intro.html. Californian Academy of Sciences, San Fransisco.

Eschmeyer, W. N. and Poss, S. G. (1976). Review of the scorpionfish genus Maxillicosta (Pisces: Scorpaenidae), with a description of three new species from the Australian-New Zealand region. Bulletin of Marine Science 26(4): 433-449.

Fourmanoir, P. and LaBoute, P. (1976). Poissons des mers tropicales Nouvelle Caledonie, Nouvelles Herbrides. Les Editions du Pacifique. 376 pp.

Fraser, T. H. (2000). A new species of Apogon (Perciformes: Apogonidae) from the Saya de Malha Bank, Indian Ocean, with redescriptions of Apogon regani Whitley, 1951, A. gardineri Regan, 1908, and A. heraldi (Herre, 1943). Proceedings of the Biological Society of Washington 113(1): 249-263.

Fraser, T. H. and Lachner, E. A. (1984). An unusual Indowest Pacific cardinalfish of the genus Apogon (Teleostei: Apogonidae). Proceedings of the Biological Society of Washington 97(3): 632-636.

Fricke, R. (1983). Revision of the Indo-Pacific genera and species of the dragonet family Callionymidae (Teleostei). Theses Zoologicae 3: 1-774.

Fricke, R. (1990). A new species of psychrolutid fish from
Western Australia. Japanese Journal of Ichthyology 36(4): 404-409.

Fricke, R. (1994). Tripterygiid fishes of Australia, New Zealand and the southwest Pacific Ocean (Teleostei). Theses Zoologicae 24: 1-585.

Fricke, R. (1997). Tripterygiid fishes of the western and central Pacific, with descriptions of 15 new species, including an annotated checklist of world Tripterygiidae (Teleostei). Koeltz Scientific Books. 607 pp.

Fricke, R. (2000). Callionymidae of New Caledonia, with remarks on related species and descriptions of 10 new species from New Caledonia, Australia, New Guinea, and Hawaii (Teleostei). Stuttgarter Beitrage zur Naturkunde Serie A (Biologie) 617: 1-81.

Garrick, J. A. F. (1982). Sharks of the genus Carcharhinus. National Oceanic and Atmospheric Administration Technical Report, National Marine Fisheries Circular 445: 1-194.

George, A. and Springer, V. G. (1980). Revision of the clinid fish tribe Ophiclinini, including five new species, and definition of the family Clinidae. Smithsonian Contributions to Zoology 307: 1-31.

Gilbert, C. H. (1905). II. The deep-sea fishes of the Hawaiian Islands. In: The aquatic resources of the Hawaiian Islands. Bulletin of theU nited States Fish Commission 23(2): 577-713.

Gill, A. C. (2004). Revision of the Indo-Pacific dottyback fish subfamily Pseudochrominae (Perciformes: Pseudochromidae). Smithiana Monographs 1: 1-213.

Gill, A. C. and Allen, G. R. (1996). Pseudochromis viridis, a new species of dottyback from Christmas Island, Indian Ocean (Teleostei: Perciformes: Pseudochromidae). Revue Française $d^{\prime}$ Aquariologie 23(1-2): 33-38.

Gill, A. C. and Allen, G. R. (2004). Pseudochromis lugubris and $P$. tonozukai, two new dottyback fish species from the Indo-Australian Archipelago (Perciformes: Pseudochromidae: Pseudochrominae). Zootaxa 604: 1-12.

Gill, A. C. and Edwards, A. J. (2002). Two new species of the Indo-Pacific fish genus Pseudoplesiops (Perciformes, Pseudochromidae, Pseudoplesiopinae). Bulletin of the Natural History Museum of London (Zoology) 68(1): 19-26.

Gill, A. C. and Hoese, D. F. (2004). Three new Australian species of the fish genus Xenisthmus (Gobioidei: Xenisthmidae). Records of the Australian Museum 56(2): 241-246.

Gill, A. C. and Hutchins, J. B. (1997). Assiculoides desmonotus, new genus and species of dottyback from the Kimberley coast of Western Australia (Teleostei: Perciformes: Pseudochromidae). Revue Française d' Aquariologie 24(1-2): 43-48.

Gill, H. S. and Miller, P. J. (1990). A new genus and species of goby from the Swan-Avon estuary, Western Australia, with a redescription of the genus Favonigobius Whitley, 1930. Records of the Western Australian Museum 14(4): 503-525.

Gill, A. C. and Mooi, R. D. (1999). Feia nota, a new species of gobiid fish from Western Australia. Records of the Western Australian Museum 19: 365-370. 
Gill, A. C., Mooi, R. D. and Hutchins, J. B. (2000). Description of a new subgenus and species of the fish genus Congrogadus Günther from Western Australia (Perciformes: Pseudochromidae). Records of the Western Australian Museum 20(1): 69-79.

Gill, A. C. and Randall, J. E. (1998). Five new species of the dottyback genus Pseudochromis from Indonesia (Teleostei: Pseudochromidae). Revue Française $d^{\prime}$ Aquariologie 25(1-2): 17-26.

Gomon, M. F. (1987). New Australian fishes. Part 6. New species of Lepidotrigla (Triglidae), Choerodon (Labridae) and Zebrias (Soleidae). Memoirs of the Museum of Victoria 48(1): 17-23.

Gomon, M. F. (2004). Two new species of roughy (Trachichthyidae: Optivus) from coastal waters of Southern Australia. Records of the Australian Museum 56(2): 173-178.

Gomon, M. F. and Allen, G. R. (1987). New Australian fishes. Part 7. A new species of Choerodon (Labridae). Memoirs of the Museum of Victoria 48(1): 25-26.

Gomon, M. F. and Paxton, J. R. (1986). A revision of the Odacidae, a temperate Australian-New Zealand labroid fish family. Indo-Pacific Fishes 8: 1-57.

Gon, O. (1993). Revision of the cardinalfish genus Cheilodipterus (Perciformes: Apogonidae), with description of five new species. Indo-Pacific Fishes 22: 1-59.

Gon, O. (1997). Revision of the cardinalfish subgenus Jaydia (Perciformes, Apogonidae, Apogon). In: Skelton and Lutjeharms, 1997, The J.L.B. Smith Institute of Ichthyology - 50 years. Transactions of the Royal Society of South Africa 51: 147-194.

Gon, O. and Allen, G. R. (1998). A new luminous cardinalfish of the genus Apogon (Perciformes: Apogonidae) from the western Pacific Ocean. J. L. B. Smith Institute Ichthyological Special Publications 62: 1-9.

Günther, A. (1860). Catalogue of the acanthopterygian fishes in the collection of the British Museum. 2. Squamipinnes, Cirrhitidae, Triglidae, Trachinidae, Sciaenidae, Polynemidae, Sphyraenidae, Trichiuridae, Scombridae, Carangidae, Xiphiidae. British Mus., London. Catalogue of Fishes 2: 1-548.

Günther, A. (1868). Catalogue of the fishes in the British Museum. Catalogue of the Physostomi, containing the families Heteropygii, Cyprinidae, Gonorhynchidae, Hyodontidae, Osteoglossidae, Clupeidae,... [thru]... Halosauridae, in the collection of the British Museum. Catalogue of Fishes 7: 1-512.

Günther, A. (1876). Remarks on fishes, with descriptions of new species in the British Museum, chiefly from southern seas. Annals and Magazine of Natural History (London) (Ser. 4) 17(101): 389-402.

Hardy, G. S. (1981). A redescription of the pufferfish Contusus richei (Tetraodontiformes: Tetraodontidae), and description of a second species of Contusus. NeW Zealand Journal of Zoology 8(1): 11-23.

Hardy, G. S. (1983a). A new genus and species of boarfish (Perciformes: Pentacerotidae) from Western Australia. Records of the Western Australian Museum 10(4): 373-380.

Hardy, G. S. (1983b). Revision of Australian species of
Torquigener Whitley (Tetraodontiformes: Tetraodontidae), and two new generic names for Australian puffer fishes. Journal of the Royal Society of New Zealand 13(1): 1-48.

Hardy, G. S. (1985). Revision of the Acanthoclinidae (Pisces: Perciformes), with descriptions of a new genus and five new species. New Zealand Journal of Zoology 11: 357-393.

Hardy, G. S. and Hutchins, J. B. (1981). On the validity of the pufferfish genus Omegophora Whitley (Tetraodontiformes: Tetraodontidae) with the description of a new species. Records of the Western Australian Museum 9(2): 187-201.

Heemstra, P. C. and Randall, J. E. (1979). A revision of the anthiine fish genus Sacura (Perciformes: Serranidae) with descriptions of two new species. $J$. L. B. Smith Institute Ichthyological Special Publications 20: 1-13.

Hensley, D. A. and Allen, G. R. (1977). A new species of Abudefduf (Pisces: Pomacentridae) from the IndoAustralian archipelago. Records of the Western Australian Museum 6(1): 107-118.

Hensley, D. A. and Randall, J. E. (1983). A new species of Abudefduf (Pisces: Pomacentridae) from the east coast of South Africa. Occasional Papers of the Bernice P. Bishop Museum 25(6): 1-10.

Hoese, D. F. and Allen, G. R. (1977). Signigobius biocellatus, a new genus and species of sand-dwelling coral reef gobiid fish from the western tropical Pacific. Japanese Journal of Ichthyology 23(4): 199-207.

Hoese, D. F. and Allen, G. R. (1983). A review of the gudgeon genus Hypseleotris (Pisces: Eleotridae) of Western Australia, with descriptions of three new species. Records of the Western Australian Museum 10(3): 243-261.

Hoese, D. F. and Allen, G. R. (1987). New Australian fishes part 10. A new genus and two new species of freshwater eleotridid fishes (Gobioidei) from the Kimberley region of Western Australia. Memoirs of the National Museum of Victoria 48(1): 35-42.

Hoese, D. F. and Allen, G. R. (1990). Descriptions of two new freshwater Glossogobius (Pisces: Gobiidae) from northern Papua New Guinea. Records of the Western Australian Museum Suppl. 34: 117-129.

Hoese, D. F. and Kuiter, R. H. (1984). A revision of the Australian plesiopid fish genus Paraplesiops, with notes on other Australian genera. Records of the Australian Museum 36: 7-18.

Hoese, D. F. and Larson, H. K. (1987). New Australian fishes. Part 11. A new genus and species of eleotridid (Gobioidei) from southern Australia with a discussion of relationships. Memoirs of the National Museum of Victoria 48(1): 43-50.

Hoese, D. F. and Larson, H. K. (1994). Revision of the Indo-Pacific gobiid fish genus Valenciennea, with descriptions of seven new species. Indo-Pacific Fishes 23: 1-71.

Hoese, D. F. and Reader, S. (1985). A new gobiid fish, Fusigobius duospilus, from the tropical Indo-Pacific. J. L. B. Smith Institute Ichthyological Special Publications 36: 1-9.

Hoese, D.F. and Rennis, D.S. (2006). Description of a new 
species of Heteroclinus (Blennoidei: Clinidae) fom southern Australia. Memoirs of Museum Victoria 63(1): 21-24.

Hoese, D. F. and Steene, R. (1978). Amblyeleotris randalli, a new species of gobiid fish living in association with alphaeid shrimps. Records of the Western Australian Museum 6(4): 379-389.

Holleman, W. (1982). Three new species and a new genus of tripterygiid fishes (Blenniodei) from the Indo-West Pacific Ocean. Annals of the Cape Provincial Museums (Natural History) 14(4): 109-137.

Holleman,-W. (1987). -Deseription_of_a_new genus_and species of tripterygiid fish (Perciformes: Blennioidei) from the Indo-Pacific, and the reallocation of Vauclusella acanthops Whitley, 1965. Cybium 11(2): 173-181.

Hutchins, J. B. (1974). Halophryne ocellatus, a new species of frogfish (Batrachoididae) from Western Australia. Records of the Western Australian Museum 3(2): 115-120.

Hutchins, J. B. (1976). A revision of the Australian frogfishes (Batrachoididae). Records of the Western Australian Museum 4(1): 3-43.

Hutchins, J. B. (1977). Descriptions of three new genera and eight new species of monacanthid fishes from Australia. Records of the Western Australian Museum 5(1): 3-58.

Hutchins, J. B. (1981). Description of a new species of serranid fish from Western Australia, with a key to the Australian species of Acanthistius. Records of the Western Australian Museum 8(4): 491-499.

Hutchins, J. B. (1983). Redescription of the clingfish Cochleoceps spatula (Gobiesocidae) from Western Australia and South Australia, with the description of a new species from Victoria and Tasmania. Records of the Western Australian Museum 11(1): 33-47.

Hutchins, J. B. (1984). Description of a new gobiesocid fish from south-western Australia, with a key to the species of Aspasmogaster. Records of the Western Australian Museum 11(2): 129-140.

Hutchins, J. B. (1986). Review of the monacanthid fish genus Pervagor, with descriptions of two new species. Indo-Pacific Fishes 12: 1-35.

Hutchins, J. B. (1987a). Description of a new plesiopid fish from south-western Australia, with a discussion of the zoogeography of Paraplesiops. Records of the Western Australian Museum 13(2): 231-240.

Hutchins, J. B. (1987b). New Australian fishes. Part 12. A new species of Eubalichthys (Monacanthidae). Memoirs of the National Museum of Victoria 48(1): $51-52$.

Hutchins, J. B. (1990). Description of a new mullid fish from south-western Australia, with comments on Upeneichthys lineatus. Records of the Western Australian Museum 14(4): 483-493.

Hutchins, J. B. (1991). Descriptions of three new species of gobiesocid fishes from southern Australia, with a key to the species of Cochleoceps. Records of the Western Australian Museum 15(3): 655-672.

Hutchins, J. B. (1994). Description of a new genus and species of monacanthid fish from India. Records of the Western Australian Museum 16(4): 567-574.
Hutchins, J. B. (1997). Review of the monacanthid fish genus Paramonacanthus, with descriptions of three new species. Records of the Western Australian Museum Suppl. 54: 1-57.

Hutchins, J. B. and Morrison, S. M. (1996). Pictilabrus brauni, a new species of labrid fish from Southwestern Australia. Revue Française d'Aquariologie 23(1-2): 39-42.

Hutchins, J. B. and Morrison, S. M. (2004). Five new species of the genus Alabes (Gobiesocidae: Cheilobranchinae). Records of the Australian Museum 56(2): 147-158.

Hutchins, J. B. and Smith, K. N. (1991). A catalogue of type specimens of fishes in the Western Australian Museum. Records of the Western Australian Museum Suppl. 38: 1-56.

ICSM (Intergovernmental Committee on Surveying and Mapping) (2005). Gazetteer of Australia. http:// www.ga.gov.au/map/names. Geoscience Australia, Australian Government.

Ivantsoff, W., Crowley, L. E. L. M. and Allen, G. R. (1987a). Descriptions of three new species and one subspecies of freshwater hardyhead (Pisces: Atherinidae: Craterocephalus) from Australia. Records of the Western Australian Museum 13(2): 171-188.

Ivantsoff, W., Crowley, L. E. L. M. and Allen, G. R. (1987b). Description of a new species of freshwater hardyhead Craterocephalus kailolae (Pisces: Atherinidae) from Safia, northeastern Papua New Guinea. Proceedings of the Linnaean Society of New South Wales 109(4): 331-337.

Ivantsoff, W., Unmack, P., Saeed, B. and Crowley, L. E. L. M. (1991). A redfinned blue-eye, a new species and genus of the family Pseudomugilidae from central western Queensland. Journal of the Australia and New Guinea Fishes Association 6(4): 277-282.

Iwamoto, T. and Williams, A. (1999). Grenadiers (Pisces, Gadiformes) from the continental slope of western and northwestern Australia. Proceedings of the Californian Academy of Science 51(3): 105-243.

Iwata, A., Hosoya, S. and Niimura, Y. (1998). Echinogobius hayashii, a new genus and species of Gobiidae. Ichthyological Research 45(2): 113-119.

Jenkins, A. P. and Allen, G. R. (2002). Neopomacentrus aquadulcis, a new species of damselfish (Pomacentridae) from eastern Papua New Guinea. Records of the Western Australian Museum 20: 379382.

Jenkins, A. P. and Boseto, D. (2005). Schismatogobius vitiensis, a new freshwater goby (Teleostei: Gobiidae) from the Fiji Islands. Ichthyological Exploration of Freshwaters 16(1): 75-82.

Jenkins, A. P., Buston, P. M. and Allen, G. R. (2000). Two new species of freshwater gudgeons (Eleotrididae: Mogurnda) from Lake Kutubu, Papua New Guinea. Ichthyological Exploration of Freshwaters 11(1): 4754.

Jewett, S. L. and Lachner, E. A. (1983). Seven new species of the Indo-Pacific genus Eviota (Pisces: Gobiidae). Proceedings of the Biological Society of Washington 96(4): 780-806. 
Jordan, D. S. and Seale, A. (1907). Fishes of the islands of Luzon and Panay. Bulletin of the United States Bureau of Fisheries 26: 1-48.

Kailola, P. J. (1990). A review of the freshwater forktailed catfishes (Pisces: Ariidae) of northern New Guinea, with descriptions of two new species. Records of the Western Australian Museum Suppl. 34: $1-30$.

Kailola, P. J. (2000). Six new species of fork-tailed catfishes (Pisces, Teleostei, Ariidae) from Australia and New Guinea. The Beagle (Occasional Papers of the Northern Territory Museum of Arts and Sciences) 16: 127-144.

Kailola, P. J. and Pierce, B. E. (1988). A new freshwater catfish (Pisces: Ariidae) from northern Australia. Records of the Western Australian Museum 14(1): 7389.

Karmovskaya, E. S. and Paxton, J. R. (2000). Revision of the Australian congrid eels of the genus Gnathophis (family Congridae), with descriptions of six new species. Journal of Ichthyology 40 (Suppl. 1): S1-S14.

Karnella, S. J. and Lachner, E. A. (1981). Three new species of the Eviota epiphanes group having vertical trunk bars (Pisces: Gobiidae). Proceedings of the Biological Society of Washington 94(1): 264-275.

Kishimoto, H. (1989). A new species and a new subspecies of the stargazer genus Gnathagnus from northwestern Australia. Japanese Journal of Ichthyology 36(3): 303-314.

Knapp, L. W. (1987). New Australian fishes. Part 13. Two new species of Platycephalidae. Memoirs of the National Museum of Victoria 48(1): 53-55.

Knapp, L. W. (1991). Platycephalus chauliodous, a new flathead fish from the eastern Indian Ocean (Teleostei: Platycephalidae). Proceedings of the Biological Society of Washington 104(1): 23-29.

Knapp, L. W. (1996). Review of the genus Cociella Whitley (Teleostei: Platycephalidae) with the description of three new species. Proceedings of the Biological Society of Washington 109(1): 17-33.

Knapp, L. W. and Imamura, H. (2004). Sunagocia sainsburyi, a new flathead fish (Scorpaeniformes: Platycephalidae) from northwestern Australia. Proceedings of the Biological Society of Washington 117(4): 545-550.

Knapp, L. W., Imamura, H. and Sakashita, M. (2000). Onigocia bimaculata, a new species of flathead fish (Scorpaeniformes: Platycephalidae) from the IndoPacific. J. L. B. Smith Institute Ichthyological Special Publications 64: 1-10.

Kuiter, R. H. (1986a). A new species of butterflyfish, Chelmonops curiosus, from Australia's south coast. Revue Française d' A quariologie 13(3): 73-78.

Kuiter, R. H. (1986b). A new genus and three new species of tripterygiid fishes of Australia's south coast. Revue Française d' Aquariologie 12(3) (1985): 89-96.

Kuiter, R. H. (2001). Revision of the Australian seahorses of the genus Hippocampus (Syngnathiformes: Syngnathidae) with description of nine new species. Records of the Australian Museum 53: 293-340.

Kuiter, R. H. (2004). Description of a new species of butterflyfish, Roa australis, from northwestern
Australia (Pisces: Perciformes: Chaetodontidae). Records of the Australian Museum 56(2): 167-171.

Kuiter, R. H. and Allen, G. R. (1986). A synopsis of the Australian pygmy perches (Percichthyidae), with the description of a new species. Revue Française $d^{\prime}$ Aquariologie 12(4): 109-116.

Kuiter, R. H. and Allen, G. R. (1999). Descriptions of three new wrasses (Teleostoi [sic]: Perciformes: Labridae: Paracheilinus) from Indonesia and northwestern Australia with evidence of possible hybridisation. Aqua, Journal of Ichthyology and Aquatic Biology 3(3): 119-132.

Lachner, E. A. and Karnella, S. J. (1980). Fishes of the Indo-Pacific genus Eviota with descriptions of eight new species (Teleostei: Gobiidae). Smithsonian Contributions to Zoology 315: 1-127.

Larson, H. K. (1985). A revision of the gobiid genus Bryaninops (Pisces), with a description of six new species. The Beagle (Occasional Papers of the Northern Territory Museum of Arts and Sciences) 2(1): 57-93.

Larson, H. K. (1987). A new species of Bryaninops (Pisces: Gobiidae) with notes on new records of three species of the genus. The Beagle (Occasional Papers of the Northern Territory Museum of Arts and Sciences) 4(1): 139-145.

Larson, H. K. (1990). A revision of the commensal gobiid genera Pleurosicya and Luposicya (Gobiidae), with descriptions of eight new species of Pleurosicya and discussion of related genera. The Beagle (Occasional Papers of the Northern Territory Museum of Arts and Sciences) 7(1): 1-53.

Larson, H. K. (2001). A revision of the gobiid fish genus Mugilogobius (Teleostei: Gobioidei), and its systematic placement. Records of the Western Australian Museum Suppl. 62: 1-233.

Larson, H. K. and Hoese, D. F. (2001). A new genus of small gobiid fish (Teleostei, Gobiidae) from the IndoWest Pacific, with description of two new species. The Beagle (Occasional Papers of the Northern Territory Museum of Arts and Sciences) 17: 27-36.

Larson, H. K. and Takita, T. (2004). Two new species of Periophthalmus (Teleostei: Gobiidae: Oxudercinae) from northern Australia, and a re-diagnosis of Periophthalmus novaeguineaensis. The Beagle (Occasional Papers of the Northern Territory Museum of Arts and Sciences) 20: 175-185.

Last, P. R., Balushkin, A. V. and Hutchins, J. B. (2002). Halaphritis platycephala (Notothenioidei: Bovichtidae): a new genus and species of temperate icefish from southeastern Australia. Copeia 2002(2): 433-440.

Last, P. R., Chidlow, J. A. and Compagno, L. J. V. (2006). A new wobbegong shark, Orectolobus hutchensi n. sp. (Orectolobiformes: Orectolobidae) from southwestern Australia. Zootaxa 1239: 35-48.

Last, P. R. and Gomon, M. F. (1987). New Australian fishes. Part 15. New species of Trygonoptera and Urolophus (Urolophidae). Memoirs of the National Museum of Victoria 48(1): 63-72.

Latreille, P. A. (1804). Tableau méthodique des Poissons. pp. 71-105. In: Nouveaux dictionnaire d'histoire naturelle, 1re éd., 24. Paris 1802-04. 
Lesueur, C. A. (1818). Description of several new species of North American fishes. Journal of the Academy of Natural Sciences, Philadelphia. 1(2): 222-235; 359368.

Leviton, A. E., Gibbs, R. H., Jr., Heal, E. and Dawson, C. E. (1985). Standards in herpetology and ichthyology: Part I. Standard symbolic codes for institutional resource collections in herpetology and ichthyology. Copeia 1985(3): 802-832.

Lubbock, R. and Allen, G. R. (1978). A distinctive new Anthias (Teleostei: Serranidae) from the western Pacific-Records-of the Western Australian Museum 6(2): 259-268.

Lubbock, R. and Allen, G. R. (1979). Canthigaster leoparda a new sharpnose pufferfish (Teleostei: Tetraodontidae) from the central Indo-Pacific. Revue Française $d^{\prime}$ Aquariologie 6(3): 87-90.

McCulloch, A. R. (1911). Report on the fishes obtained by the F. I. S. "Endeavour," on the coasts of New South Wales, Victoria, South Australia and Tasmania. Part I. Biological Results of the "Endeavour" 1(1): 1-87.

McCulloch, A. R. (1912). Notes on some Western Australian fishes. Records of the Western Australian Museum 1(2): 78-97.

McCulloch, A. R. (1914a). Report on some fishes obtained by the F. I. S. "Endeavour" on the coasts of Queensland, New South Wales, Victoria, Tasmania, South and South-western Australia. Part II. Biological Results of the "Endeavour" 2(3): 77-165.

McCulloch, A. R. (1914b). Notes on some Western Australian fishes. Records of the Western Australian Museum 1: 211-227.

McCulloch, A. R. (1915). Report on some fishes obtained by the F. I. S. "Endeavour" on the coasts of Queensland, New South Wales, Victoria, Tasmania, South and South-Western Australia, Part III. Biological Results of the "Endeavour" 3(3): 97-170.

McCulloch, A. R. and Waite, E. R. (1918). Some new and little-known fishes from South Australia. Records of the South Australian Museum (Adelaide) 1(1): 39-78.

McDowall, R. M. (1978). A new genus and species of galaxiid fish from Australia (Salmoniformes: Galaxiidae). Journal of the Royal Society of New Zealand 8(1): 115-124.

McEachran, J. D. and Fechhelm, J. D. (1982). A new species of skate from western Australia with comments on the status of Pavoraja Whitley, 1939 (Chondrichthyes: Rajiformes). Proceedings of the Biological Society of Washington 95(1): 1-12.

McKay, R. J. (1964). Description of a new stonefish of the family Synanceidae from Western Australia. Journal of the Royal Society of Western Australia 47(1): 8-12.

McKay, R. J. (1965). Type specimens in the Western Australian Museum (Part 5) - Pisces, p. 35-37. In: Annual report of the Western Australian Museum 1963-64, Western Australian Museum, Perth.

McKay, R. J. (1966a). Type specimens in the Western Australian Museum (Part 6) - Pisces, p. 46-47. In: Annual report of the Western Australian Museum 1964-65, Western Australian Museum, Perth.

McKay, R. J. (1966b). Studies on Western Australian sharks and rays of the family Scyliorhinidae,
Urolophidae and Torpedinidae. Journal of the Royal Society of Western Australia 49(3): 65-82.

McKay, R. J. (1969). The gunus Tandya in Western Australia, with a description of a new opisthognathid fish, Tandya reticulata sp. nov. Journal of the Royal Society of Western Australia 52(1): 1-2.

McKay, R. J. (1970). Additions to the fish fauna of Western Australia. 5. Western Australian Fisheries Bulletin 9(5): 3-24.

McKay, R. J. (1971). Two new genera and five new species of percophidid fishes (Pisces: Percophididae) from Western Australia. Journal of the Royal Society of Western Australia 54(2): 40-46.

McKay, R. J. (1980). The fishes of the family Sillaginidae from India with a description of a new species. J. Marine Biological Association of India 18(2) [1976]: 375-385.

McKay, R. J. (1985). A revision of the fishes of the family Sillaginidae. Memoirs of the Queensland Museum 22(1): 1-73.

Mees, G. F. (1960). The Uranoscopidae of Western Australia (Pisces, Perciformes). Journal of the Royal Society of Western Australia 43(2): 46-58.

Mees, G. F. (1961). Description of a new fish of the family Galaxiidae from Western Australia. Journal of the Royal Society of Western Australia 44(2): 33-38.

Mees, G. F. (1962a). The subterranean freshwater fauna of Yardie Creek Station, North West Cape, Western Australia. Journal of the Royal Society of Western Australia 45(1): 24-32.

Mees, G. F. (1962b). Additions to the fish fauna of Western Australia - 3. Western Australian Fisheries Bulletin 9(3): 23-30.

Mees, G. F. (1963). Description of a new freshwater fish of the family Theraponidae from Western Australia. Journal of the Royal Society of Western Australia 46(1): $1-4$.

Mees, G. F. (1964a). A new fish of the family Scorpaenidae from New Guinea. Zoologische Mededelingen (Leiden) 40(1): 1-4.

Mees, G. F. (1964b). Additions to the fish fauna of Western Australia-4. Western Australian Fisheries Bulletin 9(4): 31-55.

Mees, G. F. (1966). A new fish of the family Apogonidae from tropical Western Australia. Journal of the Royal Society of Western Australia 49(3): 83-84.

Milward, N. E. (1960). A new species of weed-fish of the genus Petraites from Western Australia. Western Australian Naturalist 7(5): 134-135.

Mohlmann, M. S. and Munday, P. L. (1999). Amblyeleotris arcupinna, a new species of shrimp goby from Papua New Guinea. Revue Française $d^{\prime}$ Aquariologie 26(1-2): 59-62.

Mohlmann, M. S. and Randall, J. E. (2002). Three new species of gobiid fishes of the genus Amblyeleotris from the central and western Pacific. Raffles Bulletin of Zoology 50(1): 215-226.

Mooi, R. D. and Jubb, R. N. (1996). Descriptions of two new species of the genus Pempheris (Pisces: Pempherididae) from Australia, with a provisional key to Australian species. Records of the Australian Museum 48(2): 117-130. 
Mooi, R. D. and Randall, J. E. (1991). Three new species of the genus Plesiops (Teleostei: Plesiopidae) from tropical Australian and adjacent seas. Copeia 1991(2): 373-387.

Motomura, H. (2004). Revision of the scorpinofish genus Neosebastes (Scorpaeniformes: Neosebastidae), with descriptions of five new species. Indo-Pacific Fishes 37: $1-76$.

Motomura, H., Last, P. R. and Yearsley, G. K. (2006). New species of shallow water scorpionfish (Scorpaenidae: Scorpaena) from the central coast of Western Australia. Copeia 2006 (3): 360-369.

Müller, J. and Henle, F. G. J. (1839). Systematische Beschreibung der Plagiostomen. Berlin. Plagiostomen. 200 pp.

Munroe, T. A. and Menke, S. B. (2004). Two new soleid flatfishes (Pleuronectiformes: Soleidae: Soleichthys) from Australian waters, with a re-description of Soleichthys microcephalus (Günther). Records of the Australian Museum 56(2): 247-258.

Murdy, E. O. and Ferraris, C. J. (2003). Gymnoamblyopus novaeguineae, a new genus and species of worm goby from Papua New Guinea (Gobiidae: Amblyopinae). Zootaxa 150: 1-6.

Murdy, E. O. and Ferraris, C. J. (2006). A revision of the marine eel-tailed catfish genus Euristhmus (Teleostei: Siluriformes: Plotosidae). The Beagle (Occasional Papers of the Northern Territory Museum of Arts and Sciences) 22: 77-90.

Nakabo, T. (1977). A new dragonet, Callionymus draconis, taken from Kochi Prefecture, Japan. Japanese Journal of Ichthyology 24(2): 98-100.

Nalbant, T. T. (1957). Cobitis aurata vallachica, eine neue Unterart des Balkan-Steinpeitzgers (Pisces, Cobitidae). Senckenbergiana Biologica 38(3): 209-212.

Nelson, J. S. (1983). Creedia alleni and Creedia partimsquamigera (Perciformes: Creediidae), two new marine fish species from Australia, with notes on other Australian creediids. Proceedings of the Biological Society of Washington 96(1): 29-37.

Nemeth, D. (1994). Systematics and distribution of fishes of the family Champsodontidae (Teleostei: Perciformes), with descriptions of three new species. Copeia 1994(2): 347-371.

Nielsen, J. G. (2002). Revision of the Indo-Pacific species of Neobythites (Teleostei, Ophidiidae), with 15 new species. Galathea Report 18: 1-56.

Orr, J. W., Fritzsche, R. A. and Randall, J. E. (2002). Solenostomus halimeda, a new species of ghost pipefish (Teleostei: Gasterosteiformes) from the IndoPacific, with a revised key to the known species of the family Solenostomidae. Aqua, Journal of Ichthyology and Aquatic Biology 5(3): 99-108.

Parin, N. V. and Kobyliansky, S. G. (1993). Review of the genus Maurolicus (Sternoptychidae, Stomiiformes), with re-establishing validity of five species considered junior synonyms of $M$. muelleri and descriptions of nine new species. Transactions of the P. P. Shirshov Institute of Oceanology 128: 69-107.

Paulin, C. D. (1986). A new genus and species of morid fish from shallow coastal waters of southern Australia. Memoirs of the Museum of Victoria 47(2): 201-206.
Paulin, C. D. and Moreland, J. M. (1979). Congiopodus coriaceus, a new species of pigfish, and a redescription of $C$. leucopaecilus (Richardson), from New Zealand (Pisces: Congiopodidae). New Zealand Journal of Zoology 6: 601-608.

Pavlov, A., Ivantsoff, W., Last, P. R. and Crowley, L. E. L. M. (1988). Kestratherina brevirostris, a new genus and species of silverside (Pisces: Atherinidae) with a review of atherinid marine and estuarine genera of southern Australia. Australian Journal of Marine and Freshwater Research 39: 385-397.

Paxton, J. R. (1975). Heraldia nocturna, a new genus and species of pipefish (family Syngnathidae) from eastern Australia, with comments on Maroubra perserrata Whitley. Proceedings of the Californian Academy of Science (Ser. 4) 40(15): 439-447.

Paxton, J. R., Hoese, D. F., Allen, G. R. and Hanley, J. E. (1989). Zoological Catalogue of Australia. Vol. 7. Pisces. Petromyzontidae to Carangidae. Australian Government Publishing Service, Canberra. 665 pp.

Peters, W. C. H. (1864). Berichtete über einige neue Säugethiere ... Amphibien ... und Fische ... Monatsberichte der Königlich Preussischen Akademie derWissenschaften zu Berlin 1864: 381-399.

Peters, W. C. H. (1876). Übersicht der von Hrn. Prof. Dr. K. Möbius in Mauritius und bei den Seychellen gesammelten Fische. Monatsberichte der Königlich Preussischen Akademie derWissenschaften zu Berlin 1876: $435-447$.

Pietsch, T. W. (1984). A review of the frogfish genus Rhycherus with the description of a new species from Western and South Australia. Copeia 1984(1): 68-72.

Pietsch, T. W. (2004). A new species of the anglerfish genus Lophiocharon Whitley (Lophiiformes: Antennariidae) from Australian waters. Records of the Australian Museum 56(2): 159-162.

Pietsch, T. W. and Grobecker, D. B. (1987). Frogfishes of the world: Systematics, zoogeography, and behavioral ecology. Stanford University Press. 420 pp.

Poss, S. G. and Allen, G. R. (1987). New Australian fishes. Part 18. A new species of Cocotropus (Aploactinidae). Memoirs of the Museum of Victoria 48(1): 79-82.

Poss, S. G. and Eschmeyer, W. N. (1978). Two new Australian velvetfishes, genus Paraploactis (Scorpaeniformes: Aploactinidae), with a revision of the genus and comments on the genera and species of the Aploactinidae. Proceedings of the Californian Academy of Science (Ser. 4) 41(18): 401-426.

Price, D. S. (1997). Chilatherina alleni, a new species of rainbowfish (Melanotaeniidae) from West Papua, Indonesia. Revue Française d' Aquariologie 24(3-4): 79-82.

Prince, J. D., Ivantsoff, W. and Potter, I. C. (1982). Atherinosoma wallacei, a new species of estuarine and inland water silverside (Teleostei: Atherinidae) from the Swan-Avon and Murray rivers, Western Australia. Australian Zoologist 21(1): 63-74.

Randall, H. A. and Allen, G. R. (1977). A revision of the damselfish genus Dascyllus (Pomacentridae) with the description of a new species. Records of the Australian Museum 31(9): 349-385.

Randall, J. E. (1978). A revision of the Indo-Pacific labrid 
fish genus Macropharyngodon, with descriptions of five new species. Bulletin of Marine Science 28(4): 742-770.

Randall, J. E. (1980). Revision of the fish genus Plectranthias (Serranidae: Anthiinae) with descriptions of 13 new species. Micronesica 16(1): 101-187.

Randall, J. E. (1981a). Two new Indo-Pacific labrid fishes of the genus Halichoeres, with notes on other species of the genus. Pacific Science 34(4): 415-432.

Randall, J. E. (1981b). Revision of the labrid fish genus Labropsis-with-deseriptions-of-five-new-species. Micronesica 17(1-2): 125-155, Plates 1-5.

Randall, J. E. (1983). A new fish of the genus Anthias (Perciformes: Serranidae) from the western Pacific, with notes on $A$. luzonensis. Freshwater and Marine Aquarium 6(9): 27-37.

Randall, J. E. (1984). Two new Indo-Pacific mugiloidid fishes of the genus Parapercis. Freshwater and Marine Aquarium 7(10): 47-54.

Randall, J. E. (1988a). Three new Indo-Pacific damselfishes of the genus Chromis (Pomacentridae). Memoirs of the Museum of Victoria 49(1): 73-81.

Randall, J. E. (1988b). Three new damselfishes of the genus Chromis (Perciformes: Pomacentridae) from the Indian Ocean. Revue Française $d^{\prime}$ Aquariologie 15(2): 49-56.

Randall, J. E. (1992). A review of the Labrid fishes of the genus Cirrhilabrus from Japan, Taiwan and the Mariana Islands, with description of two new species. Micronesica 25(1): 99-121.

Randall, J. E. (1994). Two new damselfishes (Perciformes: Pomacentridae) from Arabian waters. Revue Française d' Aquariologie 21(1-2): 39-48.

Randall, J. E. (1999). Revision of the Indo-Pacific labrid fishes of the genus Pseudochelinus, with descriptions of three new species. Indo-Pacific Fishes 28: 1-34.

Randall, J. E. (2002). Two new damselfishes of the genus Pomacentrus from the south-west Pacific. Aqua, Journal of Ichthyology and Aquatic Biology 5(4): 167176.

Randall, J. E. (2007). Leptachirus, a new soleid fish genus from New Guinea and northern Australia, with descriptions of eight new species. Records of the Western Australian Museum 24(1): 81-108.

Randall, J. E. and Allen, G. R. (1978). Anthias pictilis, a new serranid fish from the subtropical southwestern Pacific. Revue Française d'A Auariologie 5(2): 33-36.

Randall, J. E. and Allen, G. R. (1982). Chromis pelloura. A new species of damselfish from the northern Red Sea. Freshwater and Marine Aquarium 5(11): 15-19.

Randall, J. E. and Allen, G. R. (1987). Four new serranid fishes of the genus Epinephelus (Perciformes: Epinephelinae) from Western Australia. Records of the Western Australian Museum 13(3): 387-411.

Randall, J. E. and Allen, G. R. (1989). Pseudanthias sheni, a new serranid fish from Rowley Shoals and Scott Reef, Western Australia. Revue Française d' Aquariologie 15(3): 73-78.

Randall, J. E. and Allen, G. R. (2005). Neopomacentrus sororius, a new species of damselfish from the Indian Ocean, with description of a neotype for its sister species, N. azyron (Bleeker). Aqua, Journal of Ichthyology and Aquatic Biology 10 ( 2): 73-80.

Randall, J. E. and Allen, G. R. (2007). Aseraggodes crypticus, a new Sole (Pleuronectiformes: Soleidae) from Christmas Island, Indian Ocean. Records of the Western Australian Museum 24(1): 109-112.

Randall, J. E., Allen, G. R. and Robertson, D. R. (2003). Myripristis earlei, a new soldierfish (Beryciformes: Holocentridae) from the Marquesas and Phoenix Islands. Zoological Studies 42(3): 405-410.

Randall, J. E. and Carpenter, K. E. (1980). Three new labrid fishes of the genus Cirrhilabrus from the Philippines. Revue Française d' Aquariologie 7(1): 1726.

Randall, J. E. and Clements, K. D. (2001). Second revision of the surgeonfish genus Ctenochaetus (Perciformes: Acanthuridae), with descriptions of two new species. Indo-Pacific Fishes 32: 1-33.

Randall, J. E. and Eschmeyer, W. N. (2001). Revision of the Indo-Pacific scorpionfish genus Scorpaenopsis, with descriptions of eight new species. Indo-Pacific Fishes 34: 1-79.

Randall, J. E., Fraser, T. H. and Lachner, E. A. (1990). On the validity of the Indo-Pacific cardinalfishes Apogon aureus (Lacepède) and $A$. fleurieu (Lacepède), with description of a related new species from the Red Sea. Proceedings of the Biological Society of Washington 103(1): 39-62.

Randall, J. E. and Hayashi, M. (1990). Apogon selas, a new cardinalfish from the western Pacific. Japanese Journal of Ichthyology 36(4): 399-403.

Randall, J. E. and Hoese, D. F. (1985). Revision of the Indo-Pacific dartfishes, genus Ptereleotris (Perciformes: Gobioidei). Indo-Pacific Fishes 7: 1-36.

Randall, J. E. and Hoese, D. F. (1988). Apogon limenus, a new species of cardinalfish (Perciformes: Apogonidae) from New South Wales. Records of the Australian Museum 40: 359-364.

Randall, J. E., Ida, H. and Moyer, J. T. (1981). A review of the damselfishes of the genus Chromis from Japan and Taiwan, with description of a new species. Japanese Journal of Ichthyology 28(3): 203-242.

Randall, J. E. and Kuiter, R. H. (1982). Three new labrid fishes of the genus Coris from the western Pacific. Pacific Science 36(2): 159-173

Randall, J. E. and Kuiter, R. H. (1989). Cirrhilabrus punctatus, a new species of labroid fish from the southwestern Pacific. Revue Française $d^{\prime}$ Aquariologie 16(2): 43-50.

Randall, J. E., Lachner, E. A. and Fraser, T. H. (1985). A revision of the Indo-Pacific apogonid fish genus Pseudamia, with descriptions of three new species. Indo-Pacific Fishes 6: 1-23.

Randall, J. E. and Lubbock, R. (1981). A revision of the serranid fishes of the subgenus Mirolabrichthys (Anthiinae: Anthias), with description of five new species. Contributions in Science (Los Angeles) 333: $1-27$.

Randall, J. E. and Lubbock, R. (1982). Three new labrid fishes of the new genus Cirrhilabrus from the southwestern Pacific. Occasional Papers of the Bernice P. Bishop Museum 25(2): 25. 
Randall, J. E. and McCarthy, L. J. (1988). A new damselfish of the genus Chromis (Perciformes: Pomacentridae) from the Persian Gulf and Gulf of Oman. Revue Française $d^{\prime}$ Aquariologie 14(4): 133136.

Randall, J. E. and Smith, C. L. (1988). Two new species and a new genus of cardinalfishes (Perciformes: Apogonidae) from Rapa, South Pacific Ocean. American Museum Novitates 2926: 1-9.

Randall, J. E. and Taylor, L. R. (1988). Review of the IndoPacific fishes of the serranid genus Liopropoma, with descriptions of seven new species. Indo-Pacific Fishes 16: $1-47$

Rennis, D. S. and Hoese, D. F. (1985). A review of the genus Parioglossus, with descriptions of six new species (Pisces, Gobioidei). Records of the Australian Museum 36(3-4): 169-201.

Rennis, D. S. and Hoese, D. F. (1987). Aioliops, a new genus of ptereleotrine fish (Pisces: Gobioidei) from the tropical Indo-Pacific with descriptions of four new species. Records of the Australian Museum 39: 67-84.

Richards, W. J., Yato, T. and Last, P. R. (2003). Revision of the gurnard fish subgenus Otohime (Triglidae: Pterygotrigla). Smithiana, Publications in Aquatic Biodiversity Bulletin 2: 1-18.

Richardson, J. (1842). Contributions to the ichthyology of Australia. Annals and Magazine of Natural History (N.S.) 9(57): 207-218.

Richardson, J. (1844). Ichthyology of the voyage of $\mathrm{H}$. M. S. Erebus and Terror,... In: J. Richardson and J. E. Gray. The zoology of the voyage of H. H.S. "Erebus and Terror," under the command of Captain Sir J. C. Ross ... during the years 1839-43. London. Jansen, 2: 1-139.

Roberts, C. D. (1987). New Australian fishes. Part 19. A new species of Lepidoperca (Serranidae). Memoirs of the National Museum of Victoria 48(1): 83-84.

Roberts, C. D. (1989). A revision of New Zealand and Australian orange perches (Teleostei; Serranidae) previously referred to Lepidoperca pulchella (Waite) with description of a new species of Lepidoperca from New Zealand Journal of Natural History 23(3): $513-524$

Rüppell, W. P. E. S. (1829). Atlas zu der Reise im nördlichen Africa. Fische des Rothen Meeres. Frankfurt-am-Main. Part 2: 27-94.

Russell, B. C. (1985). Revision of the Indo-Pacific labrid fish genus Suezichthys, with descriptions of four new species. Indo-Pacific Fishes 2: 1-21.

Russell, B. C. (1986). Two new species of Parascolopsis (Pisces: Nemipteridae) from north-western Australia, Indonesia and the Philippines. The Beagle (Occasional Papers of the Northern Territory Museum of Arts and Sciences) 3(1): 137-142.

Russell, B. C. (1987). New Australian fishes. Part 20. A new species of Aplodactylus (Aplodactylidae). Memoirs of the National Museum of Victoria 48(1): 85-87.

Russell, B. C. (1988). Revision of the labrid fish genus Pseudolabrus and allied genera. Records of the Australian Museum Suppl. 9: 1-72

Russell, B. C. (2001). A new species of Pentapodus
(Teleostei: Nemipteridae) from the western Pacific. The Beagle (Occasional Papers of the Northern Territory Museum of Arts and Sciences) 17: 53-56.

Sasaki, K. (1992). Two new species of Nibea (Sciaenidae) from northern Australia and Papua New Guinea. Japanese Journal of Ichthyology 39(1): 1-7.

Sauvage, H. E. (1883). Descriptions de quelques poissons de la collection du Muséum d'histoire naturelle. Bulletin de la Société philomathique de Paris 7: 156161.

Schwarzhans, W., Møller, P. R. and Nielsen, J. G. (2005). Review of the Dinamatichthyini. (Teleostei: Bythitidae) of the Indo-West Pacific. Part 1. Diancistrus and two new genera with 26 new species. The Beagle. Records of the Museums and Art Galleries of the Northern Territory 21: 73-163.

Scott, J. K. (1976). A review of the fish genus Neoodax (Odacidae) of Western Australia with description of a closely allied new genus and species. Records of the Western Australian Museum 4(4): 349-373.

Scott, T. D. (1957). A new blenny (Tripterygiidae) and pipefish (Syngnathidae) from Kangaroo Island, South Australia. Transactions of the Royal Society of South Australia 80: 180-183.

Séret, B. (1990). Aulohalaelurus kanakorum sp.n., a new species of catshark (Carcharhiniformes, Scyliorhinidae, Atelomycterinae) from New Caledonia. Records of the Australian Museum 42(2): 127-136.

Serventy, D. L. (1937). Zoological notes on a trawling cruise in the Great Australian Bight. Journal of the Royal Society of Western Australia 23: 65-83.

Shen, S.-C. (1994). A revision of the tripterygiid fishes from coastal waters of Taiwan with descriptions of two new genera and five new species. Acta Zoologica Taiwanica 5(2): 1-32.

Shimizu, T. and Hutchins, J. B. (1987). New Australian fishes. Part 21. A new species of Centroberyx (Berycidae). Memoirs of the National Museum of Victoria 48(1): 89-90.

Shipway, B. (1950). Notes on the aquatic natural history of the lower Murchison river. Western Australian Naturalist 2(4): 73-77.

Shipway, B. (1953). Additional records of fishes occurring. in the fresh waters of Western Australia. Western Australian Naturalist 3(8): 173-177.

Smith, M. M. (1980). A review of the South African cheilodactylid fishes (Pisces, Perciformes), with descriptions of two new species. Ichthyological Bulletin of the J. L. B. Smith Institute of Ichthyology 42: 1-14.

Smith-Vaniz, W. F. (1987). The saber-toothed blennies, tribe Nemophini (Pisces: Blenniidae): an update. Proceedings of the Academy of Natural Sciences of Philadelpia 139: 1-52.

Smith-Vaniz, W. F. (2004). Descriptions of six new species of jawfishes (Opistognathidae: Opistognathus) from Australia. Records of the Australian Museum 56(2): 209-224.

Smith-Vaniz, W. F. and Jelks, H. L. (2006). Australian trevallies of the genus Pseudocaranx (Teleostei: Carangidae), with description of a new species from 
Western Australia. Memoirs of Museum Victoria 63(1): 97-106.

Smith-Vaniz, W., Satapoomin, U. and Allen, G. R. (2001). Meiacanthus urostigma, a new fangblenny from the northeastern Indian Ocean, with discussion and examples of mimicry in species of Meiacanthus (Teleostei: Blenniidae: Nemophini). Aqua, Journal of Ichthyology and Aquatic Biology 5(1): 25-43.

Springer, S. and D'Aubrey, J. D. (1972). Two new scyliorhinid sharks from the east coast of Africa with notes on related species. Investigative Report of the Oceanographic-Research Institute_of Durban 29: 1-9.

Springer, V. G. (1988). The Indo-Pacific blenniid fish genus Ecsenius. Smithsonian Contributions to Zoology 465: 1-134.

Springer, V. G. and Allen, G. R. (2004). Ecsenius caeruliventris and $E$. shirleyae, two new species of blenniid fishes from Indonesia, and new distribution records for other species of Ecsenius. Zootaxa 791: 112.

Springer, V. G. and Fraser, T. F. (1976). Synonymy of the fish families Cheilobranchidae (=Alabetidae) and Gobiesocidae, with descriptions of two new species of Alabes. Smithsonian Contributions to Zoology 234: 123.

Springer, V. G. and Larson, H. K. (1996). Pholidichthys anguis, a new species of pholidichthyid fish from Northern Territory and Western Australia. Proceedings of the Biological Society of Washington 109(2): 353-365.

Starnes, W. C. (1988). Revision, phylogeny and biogeographic comments on the circumtropical marine percoid fish family Priacanthidae. Bulletin of Marine Science 43(2): 117-203.

Steindachner, F. (1892). Über einige neue und seltene Fischarten aus der ichthyologischen sammlung des $\mathrm{k}$. k. naturhistorischen Hofmuseums. Denkschriften der Österreichischen Akademie der Wissenschaften. Wien 59(1): 357-384.

Tanaka, S. (1917). Eleven new species of fish from Japan. Dobutsugaku Zasshi 29(339): 7-12.

Valenciennes in Cuvier, G. and Valenciennes, A. (1828). Histoire naturelle des poissons. Tome second. Livre Troisième. Des poissons de la famille des perches, ou des percoïdes. 1-490 pp.

Valenciennes in Cuvier, G. and Valenciennes, A. (1830). Historie naturelle des poissons. Tome Sixième. Livre sixième. Partie I. Des Sparö̈des; Partie II. Des Ménides. 1-559 pp.

Valenciennes, A. (1832). Descriptions de plusieurs espèces nouvelles de poissons du genre Apogon. Nouvelles Annales Museum Histoire Naturelle, Paris 1: 51-60.

Valenciennes in Cuvier, G. and Valenciennes, A. (1833). Histoire naturelle des poissons. Tome neuvième. Suite du livre neuvième. Des Scombéroïdes. 1-512 pp.

Valenciennes in Cuvier, G. and Valenciennes, A. (1836). Histoire naturelle des poissons. Tome onzième. Livre treizième. De la famille des Mugilö̈des. Livre quatorzième. De la famille des Gobiö̈des. 1-506 pp.

Valenciennes in Cuvier, G. and Valenciennes, A. (1840). Histoire naturelle des poissons. Tome quinzième.
Suite du livre dix-septième. Siluroïdes. 1-540 pp.

Valenciennes in Cuvier, G. and Valenciennes, A. (1846). Histoire naturelle des poissons. Tome dix-huitième. Suite du livre dix-huitième. Cyprinoïdes. Livre dixneuvième. Des Ésoces ou Lucioïdes. 1-505 pp.

Vari, R. P. (1978). The terapon perches (Percoidei, Teraponidae). A cladistic analysis and taxonomic revision. Bulletin of the American Museum of Natural History 159(5): 175-340.

Vari, R. P. and Hutchins, J. B. (1978). New species of terapon perches (Percoidei, Teraponidae) from Australia. American Museum Novitates 2654: 1-8.

Waite, E. R. (1900). Notes on fishes from Western Australia and description of a new species. Records of the Australian Museum 3: 210-216.

Waite, E. R. (1904). Additions to the fish fauna of Lord Howe Island, No. 4. Records of the Australian Museum 5(3): 135-186.

Waite, E. R. (1905). Notes on fishes from Western Australia.-No. 3. Records of the Australian Museum 6(2): 55-82.

Watson, R. E. (1991). A provisional review of the genus Stenogobius with descriptions of a new subgenus and thirteen new species. (Pisces: Teleostei: Gobiidae). Records of the Western Australian Museum 15(3): 571-654.

Watson, R. E. (1994a). Stenogobius (Stenogobius) lachneri, a new species of freshwater goby from the Island of New Guinea (Pisces: Perciformes: Gobiidae). Tropical Fish Hobbyist 42(10): 160-165.

Watson, R. E. (1994b). Awaous (Awaous) acritosus, a new species of freshwater goby from southern New Guinea and northeastern Australia (Teleostei: Gobiidae). Ichthyological Exploration of Freshwaters 5(4): 371-376.

Watson, R. E. (1995). A new species of Sicyopus from Papua New Guinea with a redescription of Sicyopus multisquamatus (Teleostei: Gobiidae: Sicydiinae). Ichthyological Exploration of Freshwaters 6(3): 267278.

Watson, R. E. (1996). A review of Stiphodon from New Guinea and adjacent regions, with descriptions of five new species (Teleostei: Gobiidae: Sicydiinae). Revue Française d' Aquariologie 23(3-4): 113-132.

Watson, R. E. and Allen, G. R. (1999). New species of freshwater gobies from West Papua, Indonesia, Indonesia (Teleostei: Gobioidei: Sicydiinae). Aqua, Journal of Ichthyology and Aquatic Biology 3(3): 113118.

Watson, R. E., Allen, G. R. and Kottelat, M. (1998). A review of Stiphodon from Halmahera and West Papua, Indonesia, Indonesia, with descriptions of two new species (Teleostei: Gobiidae). Ichthyological Exploration of Freshwaters 9(3): 293-304.

Weber, M. (1907). Süsswasserfische von Neu-Guinea ein Beitrag zur Frage nach dem früheren Zusammenhang von Neu-Guinea und Australien. In: Nova Guinea. Résultats de l'expédition scientifique Néerlandaise à la Nouvelle-Guinée. 5(2): 201-267.

Weber, M. (1909). Diagnosen neuer Fische der SibogaExpedition. Notes of the Leyden Museum 31(4): 143169. 
Weber, M. (1911). Die Fische der Aru- und Kei-Inseln. Ein Beitrag zur Zoolographie dieser Inseln. Abhandlungen der Senckenbergischen Naturforschenden Gesellschaft, Frankfurt 34: 1-49.

Weber, M. and de Beaufort, L. F. (1922). The fishes of the Indo-Australian Archipelago. IV. Heteromi, Solenichthyes, Synentognathi, Percesoces, Labyrinthici, Microcyprini. E. J. Brill, Leiden. v. 4: 1410.

Wellington, G. M. (1992). Xyrichtys victori, a new species of razorfish from the Galápagos Islands (Teleostei: Labridae). Copeia 1992(4): 1053-1059.

Wellington, G. M., Allen, G. R. and Robertson, D. R. (1994). Xyrichtys perlas (Labridae), a new species of razorfish from the tropical eastern Pacific. Revue Française d' Aquariologie 21(1-2): 49-52.

White, J. (1790). Journal of a voyage to New South Wales with sixty-five plates of non descript animals, birds, lizards, serpents, curious cones of trees and other natural productions. $297 \mathrm{pp}$.

Whitley, G. P. (1931). Studies in ichthyology. No. 5. Records of the Australian Museum 18(4): 138-160.

Whitley, G. P. (1934). Studies in ichthyology. No. 8. Records of the Australian Museum 19(2): 153-163.

Whitley, G. P. (1943a). A new Australian shark. Records of the South Australian Museum (Adelaide) 7(4): 397399.

Whitley, G. P. (1943b). Ichthyological notes and illustrations. (Part 2). Australian Zoologist 10(2): 167187.

Whitley, G. P. (1944a). New sharks and fishes from Western Australia. Australian Zoologist 10(3): 252273.

Whitley, G. P. (1944b). Illustrations of some Western Australian fishes. Proceedings of the Royal Zoological Society of New South Wales 1944: 25-29.

Whitley, G. P. (1945). New sharks and fishes from Western Australia. Part 2. Australian Zoologist 11(1): $1-42$.

Whitley, G. P. (1947). New sharks and fishes from Western Australia. Part 3. Australian Zoologist 11(2): 129-150.

Whitley, G. P. (1948). New sharks and fishes from Western Australia. Part 4. Australian Zoologist 11(3): 259-276.

Whitley, G. P. (1951). New fish names and records. Proceedings of the Royal Zoological Society of New South Wales 1949-50: 61-68.

Whitley, G. P. (1955). Taxonomic notes on fishes. Proceedings of the Royal Zoological Society of New South Wales 1953-54: 44-57.

Williams, J. T. (1988). Revision and phylogenetic relationships of the blenniid fish genus Cirripectes. Indo-Pacific Fishes 17: 1-78.

Williams, J. T. (1993). Cirripectes alleni, a new species of blenniid fish from the northern coast of Western Australia (Blennioidei: Blenniidae). Revue Française $d^{\prime}$ Aquariologie 20(2): 61-64.
Williams, J. T. and Howe, J. C. (2003). Seven new species of the triplefin fish genus Helcogramma (Tripterygiidae) from the Indo-Pacific. Aqua, Journal of Ichthyology and Aquatic Biology 7(4): 151-176.

Williams, J. T. and Maugé, L. A. (1984). Cirripectes chelomatus, a new species of salarine fish (Pisces, Blenniidae). Bulletin de Museum national d'Histoire naturelle 5(4): 1139-1149.

Winterbottom, R. (1984). A review of the gobiid fish genus Trimma from the Chagos Archipelago, central Indian Ocean, with the description of seven new species. Canadian Journal of Zoology 62(4): 695-715.

Winterbottom, R. (1985). Two new gobiid fish species (in Priolepis and Trimma) from the Chagos Archipelago, central Indian Ocean. Canadian Journal of Zoology 63(4): 748-754.

Winterbottom, R. (2000). Four new species of Trimma (Gobiidae), from the Indian and western Pacific Oceans. Aqua, Journal of Ichthyology and Aquatic Biology 4(2): 57-66.

Winterbottom, R. (2001). Two new gobiid fish species in Trimma and Trimmatom (Teleostei: Gobiidae) from the Indian and Western Pacific Oceans. Aqua, Journal of Ichthyology and Aquatic Biology 5(1): 19-24.

Winterbottom, R. and Burridge, M. (1992). Revision of Egglestonichthys and of Priolepis species possessing a transverse pattern of cheek papillae (Teleostei; Gobiidae), with a discussion of relationships. Canadian Journal of Zoology 70: 1934-1946.

Winterbottom, R. and Hoese, D. F. (1988). A new genus and four new species of fishes from the Indo-West Pacific (Pisces; Perciformes; Gobiidae), with comments on relationships. Royal Ontario Museum of Life Sciences Occasional Papers 37: 1-17.

Winterbottom, R. and Villa, C. A. (2003). A new species of the Trimma caesiura species complex (Teleostei: Gobiidae) from the north-eastern margin of the Australian Plate, with a redescription of the other nominal species in the complex. Aqua, Journal of Ichthyology and Aquatic Biology 7(1): 13-28.

Wongratana, T. (1987). Four new species of clupeoid fishes (Clupeidae and Engraulidae) from Australian waters. Proceedings of the Biological Society of Washington 100(1): 104-111.

Woodland, D. J. and Allen, G. R. (1977). Siganus trispilos, a new species of Siganidae from the eastern Indian Ocean. Copeia 1977(4): 617-620.

Yamanoue, Y. and Matsuura, K. (2001). Description of two new acropomatid species of the genus Malakichthys (Teleostei: Perciformes) from Australia. Bulletin of Marine Science 69(3): 1139-1147.

Yearsley, G. K., Last, P. R. and Morris, G. B. (1997). Codes for Australian Aquatic Biota (CAAB): an upgraded and expanded species coding system for Australian fisheries databases. CSIRO Marine Laboratory Report 224.

Zaiser, M. J. and Fricke, R. (1985). Synchiropus moyeri, a new species of dragonet (Callionymidae) from Miyake-jima, Japan. Japanese Journal of Ichthyology 31(4): 389-397. 